\title{
Observational Evidence from Supernovae for an Accelerating Universe and a Cosmological Constant
}

\section{Citation}

Riess, Adam G., Alexei V. Filippenko, Peter Challis, Alejandro Clocchiatti, Alan Diercks, Peter M. Garnavich, Ron L. Gilliland, et al. 1998. “Observational Evidence from Supernovae for an Accelerating Universe and a Cosmological Constant." The Astronomical Journal 116 (3): 100938. https://doi.org/10.1086/300499.

\section{Permanent link}

http://nrs.harvard.edu/urn-3:HUL.InstRepos:41399831

\section{Terms of Use}

This article was downloaded from Harvard University's DASH repository, and is made available under the terms and conditions applicable to Other Posted Material, as set forth at http:// nrs.harvard.edu/urn-3:HUL.InstRepos:dash.current.terms-of-use\#LAA

\section{Share Your Story}

The Harvard community has made this article openly available. Please share how this access benefits you. Submit a story.

Accessibility 
The Astronomical Journal, 116:1009-1038, 1998 September

C 1998. The American Astronomical Society. All rights reserved. Printed in U.S.A.

\title{
OBSERVATIONAL EVIDENCE FROM SUPERNOVAE FOR AN ACCELERATING UNIVERSE AND A COSMOLOGICAL CONSTANT
}

\author{
Adam G. Riess, ${ }^{1}$ Alexei V. Filippenko, ${ }^{1}$ Peter Challis, ${ }^{2}$ Alejandro Clocchiatti, ${ }^{3}$ Alan Diercks, ${ }^{4}$ \\ Peter M. Garnavich, ${ }^{2}$ Ron L. Gilliland, ${ }^{5}$ Craig J. Hogan, ${ }^{4}$ Saurabh Jha, ${ }^{2}$ Robert P. Kirshner, ${ }^{2}$ \\ B. Leibundgut, ${ }^{6}$ M. M. Phillips, ${ }^{7}$ David Reiss, ${ }^{4}$ Brian P. Schmidt, ${ }^{8,9}$ Robert A. Schommer, ${ }^{7}$ \\ R. Chris SMith, ${ }^{7,10}$ J. SPyromilio, ${ }^{6}$ Christopher Stubbs, ${ }^{4}$ \\ NiCHOLAS B. SUNTZEFF, ${ }^{7}$ AND JOHN TONRY ${ }^{11}$ \\ Received 1998 March 13; revised 1998 May 6
}

\begin{abstract}
We present spectral and photometric observations of 10 Type Ia supernovae (SNe Ia) in the redshift range $0.16 \leq z \leq 0.62$. The luminosity distances of these objects are determined by methods that employ relations between SN Ia luminosity and light curve shape. Combined with previous data from our High- $z$ Supernova Search Team and recent results by Riess et al., this expanded set of 16 high-redshift supernovae and a set of 34 nearby supernovae are used to place constraints on the following cosmological parameters: the Hubble constant $\left(H_{0}\right)$, the mass density $\left(\Omega_{M}\right)$, the cosmological constant (i.e., the vacuum energy density, $\left.\Omega_{\Lambda}\right)$, the deceleration parameter $\left(q_{0}\right)$, and the dynamical age of the universe $\left(t_{0}\right)$. The distances of the high-redshift SNe Ia are, on average, $10 \%-15 \%$ farther than expected in a low mass density $\left(\Omega_{M}=0.2\right)$ universe without a cosmological constant. Different light curve fitting methods, SN Ia subsamples, and prior constraints unanimously favor eternally expanding models with positive cosmological constant (i.e., $\Omega_{\Lambda}>0$ ) and a current acceleration of the expansion (i.e., $q_{0}<0$ ). With no prior constraint on mass density other than $\Omega_{M} \geq 0$, the spectroscopically confirmed SNe Ia are statistically consistent with $q_{0}<0$ at the $2.8 \sigma$ and $3.9 \sigma$ confidence levels, and with $\Omega_{\Lambda}>0$ at the $3.0 \sigma$ and $4.0 \sigma$ confidence levels, for two different fitting methods, respectively. Fixing a "minimal" mass density, $\Omega_{M}=$ 0.2 , results in the weakest detection, $\Omega_{\Lambda}>0$ at the $3.0 \sigma$ confidence level from one of the two methods. For a flat universe prior $\left(\Omega_{M}+\Omega_{\Lambda}=1\right)$, the spectroscopically confirmed SNe Ia require $\Omega_{\Lambda}>0$ at $7 \sigma$ and $9 \sigma$ formal statistical significance for the two different fitting methods. A universe closed by ordinary matter (i.e., $\Omega_{M}=1$ ) is formally ruled out at the $7 \sigma$ to $8 \sigma$ confidence level for the two different fitting methods. We estimate the dynamical age of the universe to be $14.2 \pm 1.7 \mathrm{Gyr}$ including systematic uncertainties in the current Cepheid distance scale. We estimate the likely effect of several sources of systematic error, including progenitor and metallicity evolution, extinction, sample selection bias, local perturbations in the expansion rate, gravitational lensing, and sample contamination. Presently, none of these effects appear to reconcile the data with $\Omega_{\Lambda}=0$ and $q_{0} \geq 0$.
\end{abstract}

Key words: cosmology: observations — supernovae: general

\footnotetext{
${ }^{1}$ Department of Astronomy, University of California at Berkeley, Berkeley, CA 94720-3411.

${ }^{2}$ Harvard-Smithsonian Center for Astrophysics, 60 Garden Street, Cambridge, MA 02138.

${ }^{3}$ Departamento de Astronomía y Astrofísica, Pontificia Universidad Católica, Casilla 104, Santiago 22, Chile.

${ }^{4}$ Department of Astronomy, University of Washington, Box 351580, Seattle, WA 98195.

${ }^{5}$ Space Telescope Science Institute, 3700 San Martin Drive, Baltimore, MD 21218.

${ }^{6}$ European Southern Observatory, Karl-Schwarzschild-Strasse 2, D-85748 Garching bei München, Germany.

${ }^{7}$ Cerro Tololo Inter-American Observatory, National Optical Astronomy Observatories, Casilla 603, La Serena, Chile. NOAO is operated by the Association of Universities for Research in Astronomy, Inc., under cooperative agreement with the National Science Foundation.

${ }^{8}$ Mount Stromlo and Siding Spring Observatories, Private Bag, Weston Creek, ACT 2611, Australia.

${ }_{9}^{9}$ Visiting Astronomer, Cerro Tololo Inter-American Observatory.

${ }^{10}$ Department of Astronomy, University of Michigan, 834 Dennison Building, Ann Arbor, MI 48109.

${ }^{11}$ Institute for Astronomy, University of Hawaii, 2680 Woodlawn Drive, Honolulu, HI 96822.
}

\section{INTRODUCTION}

This paper reports observations of 10 new high-redshift Type Ia supernovae ( $\mathrm{SNe}$ Ia) and the values of the cosmological parameters derived from them. Together with the four high-redshift supernovae previously reported by our High- $z$ Supernova Search Team (Schmidt et al. 1998; Garnavich et al. 1998a) and two others (Riess et al. 1998b), the sample of 16 is now large enough to yield interesting cosmological results of high statistical significance. Confidence in these results depends not on increasing the sample size but on improving our understanding of systematic uncertainties.

The time evolution of the cosmic scale factor depends on the composition of mass-energy in the universe. While the universe is known to contain a significant amount of ordinary matter, $\Omega_{M}$, which decelerates the expansion, its dynamics may also be significantly affected by more exotic forms of energy. Preeminent among these is a possible energy of the vacuum $\left(\Omega_{\Lambda}\right)$, Einstein's "cosmological con- 
stant," whose negative pressure would do work to accelerate the expansion (Carroll, Press, \& Turner 1992; Schmidt et al. 1998). Measurements of the redshift and apparent brightness of SNe Ia of known intrinsic brightness can constrain these cosmological parameters.

\subsection{The High-z Program}

Measurement of the elusive cosmic parameters $\Omega_{M}$ and $\Omega_{\Lambda}$ through the redshift-distance relation depends on comparing the apparent magnitudes of low-redshift SNe Ia with those of their high-redshift cousins. This requires great care to assure uniform treatment of both the nearby and distant samples.

The High-z Supernova Search Team has embarked on a program to measure supernovae at high redshift and to develop the comprehensive understanding of their properties required for their reliable use in cosmological work. Our team pioneered the use of supernova light curve shapes to reduce the scatter about the Hubble line from $\sigma \approx 0.4$ mag to $\sigma \approx 0.15 \mathrm{mag}$ (Hamuy et al. 1996a, 1996c, 1995; Riess, Press, \& Kirshner 1995, 1996a). This dramatic improvement in the precision of SNe Ia as distance indicators increases the power of statistical inference for each object by an order of magnitude and sharply reduces their susceptibility to selection bias. Our team has also pioneered methods for using multicolor observations to estimate the reddening to each individual supernova, near and far, with the aim of minimizing the confusion between effects of cosmology and dust (Riess et al. 1996a; Phillips et al. 1998). Because the remaining scatter about the Hubble line is so small, the discussion of the Hubble constant from lowredshift $\mathrm{SNe}$ Ia has already passed into a discussion of the best use of Cepheid distances to galaxies that have hosted SNe Ia (Saha et al. 1997; Kochanek 1997; Madore \& Freedman 1998; Riess et al. 1996a; Hamuy et al. 1996c; Branch 1998). As the use of SNe Ia for measuring $\Omega_{M}$ and $\Omega_{\Lambda}$ progresses from its infancy into childhood, we can expect a similar shift in the discussion from results limited principally by statistical errors to those limited by our depth of understanding of SNe Ia.

Published high-redshift SN Ia data are a small fraction of the data in hand both for our team and for the Supernova Cosmology Project (Perlmutter et al. 1995, 1997, 1998). Now is an opportune time to spell out details of the analysis, since further increasing the sample size without scrupulous attention to photometric calibration, uniform treatment of nearby and distant samples, and an effective way to deal with reddening will not be profitable. Besides presenting results for four high- $z$ supernovae, we have published details of our photometric system (Schmidt et al. 1998) and stated precisely how we used ground-based photometry to calibrate our Hubble Space Telescope (HST) light curves (Garnavich et al. 1998b). In this paper, we spell out details of newly observed light curves for 10 objects, explain the recalibration of the relation of light curve shape and luminosity for a large low-redshift sample, and combine all the data from our team's work to constrain cosmological parameters. We also evaluate how systematic effects could alter the conclusions. While some comparison with the stated results of the Supernova Cosmology Project (Perlmutter et al. 1995, 1997, 1998) is possible, an informed combination of the data will have to await a similarly detailed description of their measurements.

\subsection{A Brief History of Supernova Cosmology}

While this paper emphasizes new data and constraints for cosmology, a brief summary of the subject may help readers connect work on supernovae with other approaches to measuring cosmological parameters.

Empirical evidence for SNe I presented by Kowal (1968) showed that these events had a well-defined Hubble diagram whose intercept could provide a good measurement of the Hubble constant. Subsequent evidence showed that the original spectroscopic class of Type I should be split (Doggett \& Branch 1985; Uomoto \& Kirshner 1985; Wheeler \& Levreault 1985; Wheeler \& Harkness 1990; Porter \& Filippenko 1987). The remainder of the original group, now called Type Ia, had peak brightness dispersions of $0.4 \mathrm{mag}$ to $0.6 \mathrm{mag}$ (Tammann \& Leibundgut 1990; Branch \& Miller 1993; Miller \& Branch 1990; Della Valle \& Panagia 1992; Rood 1994; Sandage \& Tammann 1993; Sandage et al. 1994). Theoretical models suggested that these "standard candles" arise from the thermonuclear explosion of a carbon-oxygen white dwarf that has grown to the Chandrasekhar mass (Hoyle \& Fowler 1960; Arnett 1969; Colgate \& McKee 1969). Because SNe Ia are so luminous $\left(M_{B} \approx-19.5 \mathrm{mag}\right)$, Colgate (1979) suggested that observations of SNe Ia at $z \approx 1$ with the forthcoming Space Telescope could measure the deceleration parameter, $q_{0}$.

From a methodical CCD-based supernova search that spaced observations across a lunation and employed prescient use of image-subtraction techniques to reveal new objects, Hansen, Jørgensen, \& Nørgaard-Nielsen (1987) detected SN 1988U, a SN Ia at $z=0.31$ (Nørgaard-Nielsen et al. 1989). At this redshift and distance precision $(\sigma \approx 0.4$ to $0.6 \mathrm{mag}$ ), $\sim 100 \mathrm{SNe}$ Ia would have been needed to distinguish between an open and a closed universe. Since the Danish group had already spent 2 years to find one object, it was clear that larger detectors and faster telescopes needed to be applied to this problem.

Evidence of systematic problems also lurked in supernova photometry, so that merely increasing the sample would not be adequate. Attempts to correct supernova magnitudes for reddening by dust (Branch \& Tammann 1992) based on the plausible (but incorrect) assumption that all SNe Ia have the same intrinsic color had the unfortunate effect of increasing the scatter about the Hubble line or alternately attributing bizarre properties to the dust absorbing SN Ia light in other galaxies. In addition, wellobserved supernovae such as SN 1986G (Phillips et al. 1987; Cristiani et al. 1992), SN 1991T (Filippenko et al. 1992a; Phillips et al. 1992; Ruiz-Lapuente et al. 1992), and SN 1991bg (Filippenko et al. 1992b; Leibundgut et al. 1993; Turatto et al. 1996) indicated that large and real inhomogeneity was buried in the scatter about the Hubble line.

Deeper understanding of low-redshift supernovae greatly improved their cosmological utility. Phillips (1993) reported that the observed peak luminosity of $\mathrm{SNe}$ Ia varied by a factor of 3. But he also showed that the decrease in $B$ brightness in the 15 days after peak $\left[\Delta m_{15}(B)\right]$ was a good predictor of the SN Ia luminosity, with slowly declining supernovae more luminous than those which fade rapidly.

A more extensive database of carefully and uniformly observed SNe Ia was needed to refine the understanding of SN Ia light curves. The Calán/Tololo survey (Hamuy et al. 1993a) made a systematic photographic search for supernovae between cycles of the full Moon. This search was 
extensive enough to guarantee the need for scheduled follow-up observations, which were supplemented by the cooperation of visiting observers, to collect well-sampled light curves. Analysis of the Calán/Tololo results generated a broad understanding of SNe Ia and demonstrated their remarkable distance precision (after template fitting) of $\sigma \approx 0.15$ mag (Hamuy et al. 1995, 1996a, 1996b, 1996c, 1996d; Tripp 1997, 1998). A parallel effort employed data from the Calán/Tololo survey and from the HarvardSmithsonian Center for Astrophysics (CfA) to develop detailed empirical models of SN Ia light curves (Riess et al. 1995; Riess 1996). This work was extended into the multicolor light curve shape (MLCS) method, which employs up to four colors of SN Ia photometry to yield excellent distance precision $(\approx 0.15 \mathrm{mag})$ and a statistically valid estimate of the uncertainty for each object with a measurement of the reddening by dust for each event (Riess et al. 1996a; see Appendix of this paper). This work has also placed useful constraints on the nature of dust in other galaxies (Riess et al. 1996b; but see Tripp 1998).

The complete sample of nearby SNe Ia light curves from the Calán/Tololo and CfA samples provides a solid foundation from which to extend the redshift-distance relation to explore cosmological parameters. The low-redshift sample used here has $34 \mathrm{SNe}$ Ia with $z<0.15$.

Since the high-redshift observations reported here consumed large amounts of observing time at the world's finest telescopes, we have a strong incentive to find efficient ways to use the minimum set of observations to derive the distance to each supernova. A recent exploration of this by Riess et al. (1998b) is the "snapshot" method, which uses only a single spectrum and a single set of photometric measurements to infer the luminosity distance to a $\mathrm{SN}$ Ia with $\sim 10 \%$ precision. In this paper, we employ the snapshot method for six SNe Ia with sparse data, but a shrewdly designed program that was intended to use the snapshot approach could be even more effective in extracting useful results from slim slices of observing time.

Application of large-format CCDs and sophisticated image analysis techniques by the Supernova Cosmology Project (Perlmutter et al. 1995) led to the discovery of SN 1992 bi $(z=0.46)$, followed by six more SNe Ia at $z \approx 0.4$ (Perlmutter et al. 1997). Employing a correction for the luminosity/light curve shape relation (but none for host galaxy extinction), comparison of these $\mathrm{SNe}$ Ia to the Calán/Tololo sample gave an initial indication of "low" $\Omega_{\Lambda}$ and "high" $\Omega_{M}: \Omega_{\Lambda}=0.06_{-0.34}^{+0.28}$ for a flat universe and $\Omega_{M}=0.88_{-0.60}^{+0.69}$ for a universe without a cosmological constant $\left(\Omega_{\Lambda} \equiv 0\right)$. The addition of one very high redshift $(z=0.83)$ SN Ia observed with HST had a significant effect on the results: $\Omega_{\Lambda}=0.4 \pm 0.2$ for a flat universe, and $\Omega_{M}=$ $0.2 \pm 0.4$ for a universe with $\Omega_{\Lambda} \equiv 0$. (Perlmutter et al. 1998). This illustrates how young and volatile the subject is at present.

\subsection{This Paper}

Our own High-z Supernova Search Team has been assiduously discovering high-redshift supernovae, obtaining their spectra, and measuring their light curves since 1995 (Schmidt et al. 1998). The goal is to provide an independent set of measurements that uses our own techniques and compares our data at high and low redshifts to constrain the cosmological parameters. Early results from four SNe Ia (three observed with $H S T$ ) hinted at a non-negligible cosmological constant and "low" $\Omega_{M}$ but were limited by statistical errors: $\Omega_{\Lambda}=0.65 \pm 0.3$ for a flat universe, $\Omega_{M}=$ $-0.1 \pm 0.5$ when $\Omega_{\Lambda} \equiv 0$ (Garnavich et al. 1998a). Our aim in this paper is to move the discussion forward by increasing the data set from four high-redshift SNe to 16 , to spell out exactly how we have made the measurement, and to consider various possible systematic effects.

In $\S 2$ we describe the observations of the SNe Ia including their discovery, spectral identification, photometric calibration, and light curves. We determine the luminosity distances (including $K$-corrections) via two methods, MLCS and a template-fitting method $\left[\Delta m_{15}(B)\right]$, as explained in $\S 3$. Statistical inference of the cosmological parameters including $H_{0}, \Omega_{M}, \Omega_{\Lambda}, q_{0}, t_{0}$, and the fate of the universe is contained in $\S 4$. Section 5 presents a quantitative discussion of systematic uncertainties that could affect our results: evolution, absorption, selection bias, a local void, weak lensing, and sample contamination. Our conclusions are summarized in $\S 6$.

\section{OBSERVATIONS}

\subsection{Discovery}

We have designed a search program to find supernovae in the redshift range $0.3<z<0.6$ with the purpose of measuring luminosity distances to constrain cosmological parameters (Schmidt et al. 1998). Distances are measured with the highest precision from $\mathrm{SNe}$ Ia observed before maximum brightness and in the redshift range of $0.35<z<0.55$, where our set of custom passbands measures the supernova light emitted in rest-frame $B$ and $V$. By imaging fields near the end of a dark run, and then again at the beginning of the next dark run, we ensure that the newly discovered supernovae are young (Nørgaard-Nielsen et al. 1989; Hamuy et al. 1993a; Perlmutter et al. 1995). Observing a large area and achieving a limiting magnitude of $m_{R} \approx 23$ mag yields many SN Ia candidates in the desired redshift range (Schmidt et al. 1998). By obtaining spectra of these candidates with $4 \mathrm{~m}$ to $10 \mathrm{~m}$ telescopes, we can identify the SNe Ia and confirm their youth using the spectral feature aging technique of Riess et al. (1997).

The 10 new SNe Ia presented in this paper (SN 1995ao, SN 1995ap, SN 1996E, SN 1996H, SN 1996I, SN 1996J, SN 1996K, SN 1996R, SN 1996T, and SN 1996U) were discovered using the CTIO $4 \mathrm{~m}$ Blanco Telescope with the facility prime-focus CCD camera as part of a three-night program in 1995 October-November and a six-night program in 1996 February-March. This instrument has a pixel scale of 0.43 , and the Tek $2048 \times 2048$ pixel CCD frame covers $0.06 \mathrm{deg}^{2}$. In each of the search programs, multiple images were combined after removing cosmic rays, differenced with "template" images, and searched for new objects using the prescription of Schmidt et al. (1998). The data on 1995 October 27 and November 17 were gathered under mediocre conditions, with most images having seeing worse than 1 ..5. The resulting differenced images were sufficient to find new objects brighter than $m_{R}=22.5 \mathrm{mag}$. The data acquired in 1996 had better image quality $(\sim 1$ 1.5), and the differenced images were sufficient to uncover new objects brighter than $m_{R}=23 \mathrm{mag}$.

In total, 19 objects were identified as possible supernovae - two new objects were detected on each of 1995 November 17 and 1995 November 29, five new objects on 1996 February 14-15, two on 1996 February 20-21, and eight on 1996 March 15-16 (Kirshner et al. 1995; Garnavich et al. 1996a, 1996b). 


\subsection{Data}

Spectra of the supernova candidates were obtained to classify the SNe and obtain redshifts of their host galaxies. For this purpose, the Keck Telescope, Multiple Mirror Telescope (MMT), and the European Southern Observatory $3.6 \mathrm{~m}$ (ESO $3.6 \mathrm{~m}$ ) were utilized following the fall of 1995 and spring of 1996 search campaigns. Some galaxy redshifts were obtained with the Keck Telescope in the spring of 1998.

The Keck spectra were taken with the Low Resolution Imaging Spectrograph (LRIS; Oke et al. 1995), providing a resolution of $6 \AA$ full width at half-maximum (FWHM). Exposure times were between $3 \times 900$ and $5 \times 900 \mathrm{~s}$, depending on the candidate brightness.

The MMT spectra were obtained with the Blue Channel Spectrograph and 500 line $\mathrm{mm}^{-1}$ grating, giving a resolution of $3.5 \AA$ FWHM. Exposure times were $1200 \mathrm{~s}$ and repeated five to seven times. The MMT targets were placed on the slit using an offset from a nearby bright star.

The ESO $3.6 \mathrm{~m}$ data were collected with the ESO Faint Object Spectrograph Camera (EFOSC1) at a nominal resolution of $18 \AA$ FWHM. Single $2700 \mathrm{~s}$ exposures were made of each target.

Using standard reduction packages in IRAF, the CCD images were bias-subtracted and divided by a flat-field frame created from a continuum lamp exposure. Multiple images of the same object were shifted where necessary and combined using a median algorithm to remove cosmic-ray events. For single exposures, cosmic rays were removed by hand using the IRAF/IMEDIT routine. Sky emission lines were problematic, especially longward of $8000 \AA$. The spectra were averaged perpendicular to the dispersion direction, and that average was subtracted from each line along the dispersion. However, residual noise from the sky lines remains. The one-dimensional spectra were then extracted using the IRAF/APSUM routine and wavelength-calibrated either from a comparison lamp exposure or the sky emission lines. The flux was calibrated using observations of standard stars and the IRAF/ONEDSTDS database.

The candidates were classified from visual inspection of their spectra and comparison with the spectra of wellobserved supernovae (see $\S 5.7$ ). In all, 10 of the candidates were $\mathrm{SNe}$ Ia, one was a SN II, and two were active galactic nuclei or SNe II (Kirshner et al. 1995; Garnavich et al. 1996a, 1996b). The remaining six candidates were observed, but the spectra did not have sufficient signal to allow an unambiguous classification. The identification spectra for the 10 new SNe Ia are summarized in Table 1 and shown in Figure 1. In addition we include the spectral data for three previously analyzed SNe: SN 1997ce, SN 1997cj, and SN 1997ck (Garnavich et al. 1998a). The spectral data for SN $1995 \mathrm{~K}$ are given by Schmidt et al. (1998). The spectrum of SN 1997ck shows only an [O II] emission line at $7328.9 \AA$ in four separate exposures (Garnavich et al. 1998a). The equivalent $R$-band magnitude of the exposure was 26.5 , which is more than 1.5 mag dimmer than the supernova would have been in $R$, suggesting that the $\mathrm{SN}$ was not in the slit when the host galaxy was observed.

Most of the host galaxies showed emission lines of [O II], [O III], or $\mathrm{H} \alpha$ in the spectrum, and the redshift was easily measured for these. For the remainder, the redshift was found by matching the broad features in the high-redshift supernovae to those in local supernova spectra. The intrinsic dispersion in the expansion velocities of $\mathrm{SNe} \mathrm{Ia}$ (Branch et al. 1988; Branch \& van den Bergh 1993) limits the precision of this method to $1 \sigma \approx 2500 \mathrm{~km} \mathrm{~s}^{-1}$ independent of the signal-to-noise ratio of the $\mathrm{SN}$ spectrum. The method used to determine the redshift for each $\mathrm{SN}$ is given in Table 1.

Following the discovery and identification of the SNe Ia, photometry of these objects was obtained from observatories scheduled around the world. The SNe were primarily observed through custom passbands designed to match the wavelength range closest to rest-frame Johnson $B$ and $V$ passbands. Our "B45," "V45," "B35," and "V35" filters are specifically designed to match Johnson $B$ and $V$ redshifted by $z=0.45$ and $z=0.35$, respectively. The characteristics of these filters are described by Schmidt et al. (1998). A few observations were obtained through standard bandpasses as noted in Table 2, where we list the photometric observations for each $\mathrm{SN}$ Ia.

Photometry of local standard stars in the supernova fields in the B35, V35, B45, V45 (or "supernova") photometric system were derived from data taken on three photometric nights. The method has been described in Schmidt et

TABLE 1

High-z SUPERNOVA SPECTROSCOPY

\begin{tabular}{|c|c|c|c|c|c|}
\hline SN & UT Date & Telescope & $\begin{array}{l}\text { Spectral Range } \\
(\mathrm{nm})\end{array}$ & Redshift & Comparison $^{\mathrm{a}}$ \\
\hline $1995 a 0 \ldots . .$. & 1995 Nov 23 & Keck I & $510-1000$ & $0.24^{b}$ & $1996 X(-4)$ \\
\hline 1995ap....... & 1995 Nov 23 & Keck I & $510-1000$ & $0.30^{\mathrm{c}}$ & $1996 \times(-4)$ \\
\hline $1996 \mathrm{E} \ldots \ldots$ & 1996 Feb 23 & ESO $3.6 \mathrm{~m}$ & $600-990$ & $0.43^{\mathrm{b}}$ & 1989B(+9) \\
\hline $1996 \mathrm{H} . . . .$. & 1996 Feb 23 & ESO $3.6 \mathrm{~m}$ & $600-990$ & $0.62^{\mathrm{b}}$ & $1996 X(+5)$ \\
\hline $1996 \mathrm{I} \ldots \ldots$ & 1996 Feb 23 & ESO $3.6 \mathrm{~m}$ & $600-990$ & $0.57^{\mathrm{c}}$ & $1996 X(+5)$ \\
\hline $1996 \mathrm{~J} \ldots \ldots$. & 1996 Feb 23 & ESO $3.6 \mathrm{~m}$ & $600-990$ & $0.30^{\mathrm{b}}$ & $1995 \mathrm{D}(+0)$ \\
\hline $1996 \mathrm{~K} \ldots \ldots$ & 1996 Feb 23 & ESO $3.6 \mathrm{~m}$ & $600-990$ & $0.38^{\mathrm{c}}$ & $1995 \mathrm{D}(+0)$ \\
\hline 1996R ...... & $1996 \operatorname{Mar} 18$ & MMT & $400-900$ & $0.16^{\mathrm{b}}$ & $1989 \mathrm{~B}(+12)$ \\
\hline 1996T ........ & 1996 Mar 18 & MMT & $400-900$ & $0.24^{\mathrm{b}}$ & $1996 \times(-4)$ \\
\hline 1996U ...... & 1996 Mar 18 & MMT & $400-900$ & $0.43^{b}$ & $1995 \mathrm{D}(+0)$ \\
\hline 1997ce ....... & 1997 May 4 & Keck II & $570-940$ & $0.44^{\mathrm{c}}$ & $1995 \mathrm{D}(+0)$ \\
\hline 1997cj ...... & 1997 May 2 & MMT & $400-900$ & $0.50^{\mathrm{b}}$ & \\
\hline 1997cj ...... & 1997 May 4 & Keck II & $570-940$ & $0.50^{\mathrm{c}}$ & $1995 \mathrm{D}(+0)$ \\
\hline $1997 \mathrm{ck} \ldots \ldots$ & 1997 May 4 & Keck II & $570-940$ & $0.97^{b}$ & $\ldots$ \\
\hline
\end{tabular}

${ }^{\text {a }}$ Supernova and its age (relative to $B$ maximum) used for comparison spectrum in Fig. 1 .

${ }^{\mathrm{b}}$ Derived from emission lines in host galaxy.

${ }^{c}$ Derived from broad features in SN spectrum. 

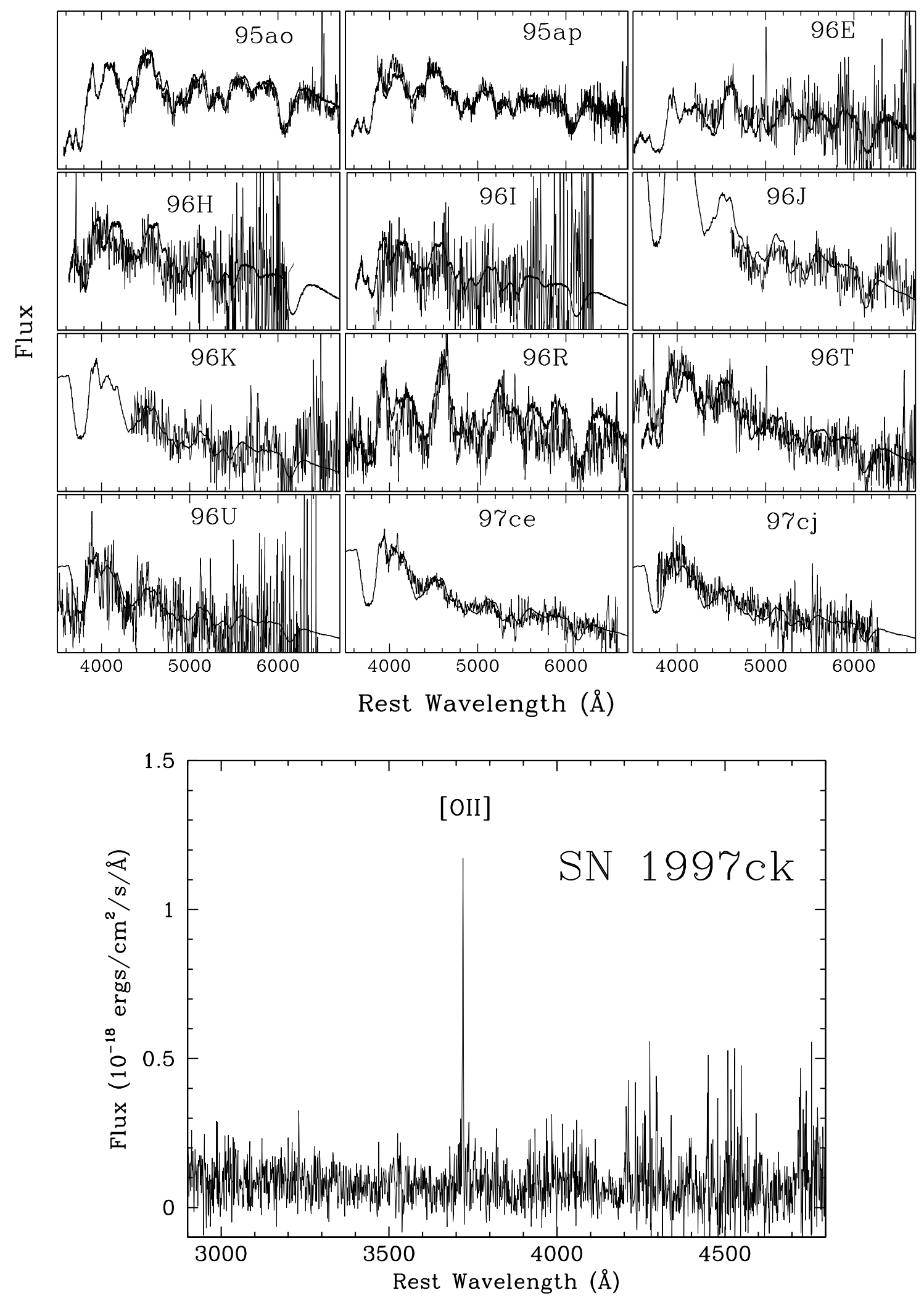

FIG. 1.-Identification spectra (in $f_{\lambda}$ ) of high-redshift SNe Ia. The spectra obtained for the 10 new SNe of the high-redshift sample are shown in the rest frame. The data are compared to nearby SN Ia spectra of the same age as determined by the light curves (see Table 1). The spectra of the three objects from Garnavich et al. (1998a) are also displayed. 
TABLE 2

SN Ia IMAgING

\begin{tabular}{|c|c|c|c|c|c|c|}
\hline $\mathrm{JD}^{\mathrm{a}}$ & UT Date & B45 & V45 & B35 & V35 & Telescope \\
\hline \multicolumn{7}{|c|}{ SN 1996E } \\
\hline $127.6 \ldots \ldots$ & 1996 Feb 14 & $22.30(0.09)$ & \multirow{3}{*}{$21.86(0.08)$} & \multirow[b]{2}{*}{$\begin{array}{l}\cdots \\
\ldots\end{array}$} & \multirow{2}{*}{$\begin{array}{l}\cdots \\
\ldots\end{array}$} & CTIO $4 \mathrm{~m}$ \\
\hline $128.6 \ldots \ldots$ & $1996 \mathrm{Feb} 15$ & $22.27(0.04)$ & & & & \multirow{2}{*}{$\begin{array}{l}\text { CTIO } 4 \mathrm{~m} \\
\text { ESO NTT }\end{array}$} \\
\hline $132.1 \ldots \ldots$ & 1996 Feb 19 & $22.46^{\mathrm{R}}(0.11)$ & & \multirow{2}{*}{$\begin{array}{l}\ldots \\
\ldots\end{array}$} & $\ldots$ & \\
\hline $134.6 \ldots \ldots$ & $1996 \mathrm{Feb} 21$ & $22.66(0.10)$ & $21.99(0.26)$ & & $\ldots$ & CTIO 4 m \\
\hline $135.5 \ldots \ldots$ & 1996 Feb 22 & $22.68(0.13)$ & \multirow{2}{*}{$\begin{array}{l}22.09(0.06) \\
22.29(0.15)\end{array}$} & $\ldots$ & $\ldots$ & CTIO 4 m \\
\hline $138.7 \ldots \ldots$ & $1996 \mathrm{Feb} 25$ & $23.04(0.12)$ & & $\ldots$ & $\ldots$ & ESO $1.5 \mathrm{~m}$ \\
\hline $139.6 \ldots \ldots$ & $1996 \mathrm{Feb} 26$ & $22.89(0.15)$ & $22.72(0.33)$ & $\ldots$ & $\ldots$ & ESO $1.5 \mathrm{~m}$ \\
\hline $157.6 \ldots \ldots$ & 1996 Mar 15 & $24.32(0.18)$ & $23.51(0.77)$ & $\ldots$ & $\ldots$ & CTIO 4 m \\
\hline $163.7 \ldots \ldots$ & 1996 Mar 21 & $\ldots$ & $22.87(0.50)$ & $\ldots$ & $\ldots$ & WIYN \\
\hline & & & SN 1996H & & & \\
\hline $127.6 \ldots \ldots$ & $1996 \mathrm{Feb} 14$ & $22.78(0.13)$ & & $\cdots$ & $\cdots$ & CTIO $4 \mathrm{~m}$ \\
\hline $128.6 \ldots \ldots$ & $1996 \mathrm{Feb} 15$ & $22.81(0.06)$ & $22.25(0.14)$ & $\ldots$ & $\ldots$ & CTIO 4 m \\
\hline $132.1 \ldots \ldots$ & $1996 \mathrm{Feb} 19$ & $22.71^{\mathrm{R}}(0.29)$ & $22.40^{1}(0.37)$ & $\ldots$ & $\ldots$ & ESO NTT \\
\hline $134.6 \ldots \ldots$ & 1996 Feb 21 & $22.85(0.08)$ & $22.48(0.19)$ & $\ldots$ & $\ldots$ & CTIO 4 m \\
\hline $135.5 \ldots \ldots$ & 1996 Feb 22 & $22.83(0.18)$ & $22.28(0.10)$ & $\ldots$ & $\ldots$ & ESO $3.6 \mathrm{~m}$ \\
\hline $136.6 \ldots \ldots$ & $1996 \mathrm{Feb} 23$ & $22.84(0.13)$ & & $\ldots$ & $\ldots$ & ESO 3.6 m \\
\hline $138.7 \ldots \ldots$ & $1996 \mathrm{Feb} 25$ & $22.85(0.09)$ & $22.58(0.15)$ & $\ldots$ & $\ldots$ & ESO $1.5 \mathrm{~m}$ \\
\hline $139.6 \ldots \ldots$ & 1996 Feb 26 & $22.88(0.15)$ & $22.52(0.25)$ & $\ldots$ & $\ldots$ & ESO $1.5 \mathrm{~m}$ \\
\hline $140.6 \ldots \ldots$ & $1996 \mathrm{Feb} 27$ & $22.96(0.16)$ & $23.10(0.10)$ & $\ldots$ & $\ldots$ & ESO $1.5 \mathrm{~m}$ \\
\hline $141.6 \ldots \ldots$ & $1996 \mathrm{Feb} 28$ & $23.05(0.08)$ & & $\ldots$ & $\ldots$ & WIYN \\
\hline $142.6 \ldots \ldots$ & $1996 \mathrm{Feb} 29$ & $23.21(0.20)$ & $22.69(0.16)$ & $\ldots$ & $\ldots$ & WIYN \\
\hline $157.6 \ldots \ldots$ & 1996 Mar 15 & $23.98(0.22)$ & $23.18(0.28)$ & $\ldots$ & $\ldots$ & CTIO 4 m \\
\hline $161.6 \ldots \ldots$ & 1996 Mar 19 & $24.16(0.22)$ & & $\ldots$ & $\ldots$ & CTIO 4 m \\
\hline $164.6 \ldots \ldots$ & 1996 Mar 22 & $\ldots$ & $24.01(0.30)$ & $\ldots$ & $\ldots$ & WIYN \\
\hline & & & SN 1996I & & & \\
\hline $128.6 \ldots \ldots$ & $1996 \mathrm{Feb} 15$ & $22.77(0.05)$ & & $\cdots$ & $\ldots$ & CTIO 4 m \\
\hline $132.1 \ldots \ldots$ & $1996 \mathrm{Feb} 19$ & $22.95(0.22)$ & $22.30(0.22)$ & $\ldots$ & $\ldots$ & ESO NTT \\
\hline $134.6 \ldots \ldots$ & 1996 Feb 21 & $22.95(0.05)$ & $22.65(0.15)$ & $\ldots$ & $\ldots$ & CTIO 4 m \\
\hline $135.5 \ldots \ldots$ & $1996 \mathrm{Feb} 22$ & $22.92(0.05)$ & $22.64(0.20)$ & $\ldots$ & $\ldots$ & ESO $3.6 \mathrm{~m}$ \\
\hline $136.6 \ldots \ldots$ & $1996 \mathrm{Feb} 23$ & $22.88(0.09)$ & $22.74(0.28)$ & $\ldots$ & $\ldots$ & ESO 3.6 m \\
\hline $138.7 \ldots \ldots$ & $1996 \mathrm{Feb} 25$ & $23.12(0.13)$ & $22.86(0.17)$ & $\ldots$ & $\ldots$ & ESO $1.5 \mathrm{~m}$ \\
\hline $140.6 \ldots \ldots$ & $1996 \mathrm{Feb} 27$ & $23.64(0.36)$ & $22.67(0.36)$ & $\ldots$ & $\ldots$ & ESO $1.5 \mathrm{~m}$ \\
\hline $142.6 \ldots \ldots$ & 1996 Feb 29 & $23.48(0.10)$ & $23.06(0.22)$ & $\ldots$ & $\ldots$ & WIYN \\
\hline $157.6 \ldots \ldots$ & 1996 Mar 15 & $24.83(0.17)$ & $23.66(0.30)$ & $\ldots$ & $\ldots$ & CTIO 4 m \\
\hline $161.6 \ldots \ldots$ & 1996 Mar 19 & $24.70(0.31)$ & $\ldots$ & $\ldots$ & $\ldots$ & CTIO 4 m \\
\hline & & & SN 1996J & & & \\
\hline $127.6 \ldots \ldots$ & $1996 \mathrm{Feb} 14$ & $22.01(0.02)$ & & & & CTIO 4 m \\
\hline $128.6 \ldots \ldots$ & $1996 \mathrm{Feb} 15$ & $21.95(0.03)$ & $21.95(0.07)$ & $\ldots$ & $\ldots$ & CTIO 4 m \\
\hline $134.6 \ldots \ldots$ & 1996 Feb 21 & $21.57(0.03)$ & $21.59(0.05)$ & & & CTIO 4 m \\
\hline $135.6 \ldots \ldots$ & $1996 \mathrm{Feb} 22$ & $21.62(0.04)$ & $21.61(0.04)$ & $21.84(0.03)$ & $21.46(0.06)$ & ESO $3.6 \mathrm{~m}$ \\
\hline $135.6 \ldots \ldots$ & 1996 Feb 22 & & & $21.89(0.04)$ & $21.47(0.02)$ & ESO 3.6 m \\
\hline $139.7 \ldots \ldots$ & $1996 \mathrm{Feb} 26$ & $21.63(0.04)$ & $21.46(0.07)$ & & & ESO $1.5 \mathrm{~m}$ \\
\hline $140.7 \ldots \ldots$ & 1996 Feb 27 & & & $21.90(0.07)$ & $21.77(0.05)$ & ESO $1.5 \mathrm{~m}$ \\
\hline $157.6 \ldots \ldots$ & 1996 Mar 15 & $22.77(0.05)$ & $22.06(0.12)$ & $23.69(0.07)$ & $21.83(0.04)$ & CTIO 4 m \\
\hline $161.8 \ldots \ldots$ & 1996 Mar 19 & $\ldots$ & $\ldots$ & $24.34(0.19)$ & $22.05(0.05)$ & CTIO 4 m \\
\hline $166.6 \ldots \ldots$ & 1996 Mar 24 & $\ldots$ & $\ldots$ & $\ldots$ & $22.76(0.07)$ & CTIO $1.5 \mathrm{~m}$ \\
\hline & & & SN 1996K & & & \\
\hline $128.5 \ldots \ldots$ & $1996 \mathrm{Feb} 15$ & $23.74(0.04)$ & & & & CTIO 4 m \\
\hline $135.5 \ldots \ldots$ & 1996 Feb 22 & $22.49(0.07)$ & $\ldots$ & $\ldots$ & $\ldots$ & ESO $3.6 \mathrm{~m}$ \\
\hline $135.5 \ldots \ldots$ & $1996 \mathrm{Feb} 22$ & $22.52(0.07)$ & $\ldots$ & $\ldots$ & $\ldots$ & ESO $3.6 \mathrm{~m}$ \\
\hline $135.7 \ldots \ldots$ & 1996 Feb 22 & $22.56(0.03)$ & $22.48(0.06)$ & $\ldots$ & $\ldots$ & ESO $3.6 \mathrm{~m}$ \\
\hline $136.6 \ldots \ldots$ & $1996 \mathrm{Feb} 23$ & $22.48(0.05)$ & $22.26(0.16)$ & $\ldots$ & $\ldots$ & ESO $1.5 \mathrm{~m}$ \\
\hline $138.6 \ldots \ldots$ & $1996 \mathrm{Feb} 25$ & $22.15(0.10)$ & $22.47(0.11)$ & $\ldots$ & $\ldots$ & ESO $1.5 \mathrm{~m}$ \\
\hline $138.7 \ldots \ldots$ & $1996 \mathrm{Feb} 25$ & $22.18(0.07)$ & &. & $\ldots$ & ESO $1.5 \mathrm{~m}$ \\
\hline $139.6 \ldots \ldots$ & $1996 \mathrm{Feb} 26$ & $22.37(0.05)$ & $22.42(0.13)$ & $\ldots$ & $\ldots$ & ESO $1.5 \mathrm{~m}$ \\
\hline $140.8 \ldots \ldots$ & $1996 \mathrm{Feb} 27$ & & & $22.23(0.10)$ & $22.06(0.11)$ & ESO $1.5 \mathrm{~m}$ \\
\hline $157.5 \ldots \ldots$ & 1996 Mar 15 & $22.83(0.07)$ & $\ldots$ & $22.93(0.12)$ & $22.61(0.19)$ & CTIO 4 m \\
\hline $157.5 \ldots \ldots$ & 1996 Mar 15 & $22.81(0.09)$ & $\ldots$ & $22.86(0.10)$ & $22.45(0.10)$ & CTIO 4 m \\
\hline $161.7 \ldots \ldots$ & 1996 Mar 19 & $23.20(0.16)$ & $22.45(0.13)$ & $23.17(0.17)$ & $22.69(0.15)$ & CTIO 4 m \\
\hline $162.6 \ldots \ldots$ & 1996 Mar 20 & $23.17(0.06)$ & $22.79(0.12)$ & & & WIYN \\
\hline $165.6 \ldots \ldots$ & 1996 Mar 23 & ... & ... & $23.58(0.16)$ & $23.17(0.14)$ & CTIO 1.m \\
\hline $168.5 \ldots \ldots$ & 1996 Mar 26 & $\ldots$ & .. & & $23.20(0.19)$ & CTIO 1.m \\
\hline $169.7 \ldots \ldots$ & 1996 Mar 27 & $24.05(0.26)$ & $\ldots$ & $24.42(0.25)$ & $\ldots$ & MDM \\
\hline
\end{tabular}


TABLE 2-Continued

\begin{tabular}{|c|c|c|c|c|c|c|}
\hline $\mathrm{JD}^{\mathrm{a}}$ & UT Date & B45 & V45 & B35 & V35 & Telescope \\
\hline \multicolumn{7}{|c|}{ SN 1996R } \\
\hline 157.7. & 1996 Mar 15 & $20.48(0.01)$ & & $\ldots$ & $\cdots$ & CTIO $4 \mathrm{~m}$ \\
\hline $158.7 \ldots \ldots$ & 1996 Mar 16 & $20.59(0.03)$ & $20.70(0.03)$ & $\ldots$ & $\ldots$ & CTIO $4 \mathrm{~m}$ \\
\hline $167.7 \ldots \ldots$ & 1996 Mar 25 & & $\ldots$ & $\ldots$ & $21.62^{\mathrm{V}}(0.04)$ & CTIO $1.5 \mathrm{~m}$ \\
\hline $191.7 \ldots \ldots$ & 1996 Apr 18 & $22.41(0.09)$ & $\ldots$ & $\ldots$ & $\ldots$ & ESO $1.5 \mathrm{~m}$ \\
\hline \multicolumn{7}{|c|}{ SN 1996T } \\
\hline $161.7 \ldots \ldots$ & 1996 Mar 19 & $20.83^{\mathrm{R}}(0.03)$ & $\ldots$ & $20.86^{\mathrm{V}}(0.02)$ & $\ldots$ & CTIO $4 \mathrm{~m}$ \\
\hline $167.6 \ldots \ldots$ & 1996 Mar 25 & $20.95^{\mathrm{R}}(0.04)$ & $\cdots$ & $20.96^{\mathrm{v}}(0.03)$ & $\ldots$ & CTIO $1.5 \mathrm{~m}$ \\
\hline $191.7 \ldots \ldots$ & 1996 Apr 18 & & $\ldots$ & $22.37 \mathrm{v}(0.17)$ & $\ldots$ & ESO $1.5 \mathrm{~m}$ \\
\hline $212.6 \ldots \ldots$ & 1996 May 9 & $22.52^{\mathrm{R}}(0.08)$ & $\cdots$ & $22.99^{\mathrm{v}}(0.31)$ & $\cdots$ & WIYN \\
\hline \multicolumn{7}{|c|}{ SN 1996U } \\
\hline 158.7 . & 1996 Mar 16 & $22.16(0.04)$ & & $\ldots$ & $\ldots$ & CTIO $4 \mathrm{~m}$ \\
\hline 160.7 . & 1996 Mar 18 & $22.00(0.11)$ & $22.03(0.18)$ & $\ldots$ & $\ldots$ & MDM \\
\hline 161.7 . & 1996 Mar 19 & $22.04(0.05)$ & $22.23(0.26)$ & $\ldots$ & $\ldots$ & CTIO $4 \mathrm{~m}$ \\
\hline $165.7 \ldots \ldots$ & 1996 Mar 23 & & $22.35(0.28)$ & $\ldots$ & $\ldots$ & CTIO $1.5 \mathrm{~m}$ \\
\hline $167.7 \ldots \ldots$ & 1996 Mar 25 & $22.19(0.10)$ & & $\ldots$ & $\ldots$ & CTIO $1.5 \mathrm{~m}$ \\
\hline $186.7 \ldots \ldots$ & 1996 Apr 13 & $23.33^{\mathrm{R}}(0.17)$ & $22.64^{\mathrm{I}}(0.28)$ & $\ldots$ & $\ldots$ & Las Campanas \\
\hline $188.7 \ldots \ldots$ & 1996 Apr 15 & $23.51(0.17)$ & $22.96(0.36)$ & $\ldots$ & $\ldots$ & WIYN \\
\hline \multicolumn{7}{|c|}{ SN 1995ao } \\
\hline $39.6 \ldots \ldots$. & 1995 Nov 18 & $21.42(0.05)$ & & $\ldots$ & $\ldots$ & CTIO 4 m \\
\hline $46.6 \ldots \ldots$. & 1995 Nov 25 & $21.30(0.03)$ & $21.10(0.13)$ & & 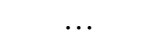 & WIYN \\
\hline $51.6 \ldots \ldots$. & 1995 Nov 30 & $21.24(0.05)$ & $\ldots$ & $21.52(0.05)$ & $21.12(0.03)$ & CTIO $4 \mathrm{~m}$ \\
\hline \multicolumn{7}{|c|}{ SN 1995ap } \\
\hline $39.6 \ldots \ldots$. & 1995 Nov 18 & $22.41(0.14)$ & & & $\cdots$ & CTIO $4 \mathrm{~m}$ \\
\hline $46.6 \ldots \ldots$. & 1995 Nov 25 & $21.13(0.08)$ & $21.40(0.10)$ & $\ldots$ & $\ldots$ & WIYN \\
\hline $48.6 \ldots \ldots \ldots$ & 1995 Nov 27 & $21.04(0.11)$ & $\ldots$ & $\ldots$ & $\ldots$ & WIYN \\
\hline $51.6 \ldots \ldots$ & 1995 Nov 30 & $21.04(0.11)$ & $\ldots$ & $21.65(0.09)$ & $20.92(0.07)$ & CTIO $4 \mathrm{~m}$ \\
\hline
\end{tabular}

NoTE.-Uncertainties in magnitudes are listed in parentheses.

${ }^{a}$ Actually JD $-2,450,000$.

al. (1998) but we summarize it here. The supernova photometric system has been defined by integrating the fluxes of spectrophotometric standards from Hamuy et al. (1994) through the supernova bandpass response functions (based on the filter transmissions and a typical CCD quantum efficiency function) and solving for the photometric coefficients that would yield zero color for these stars and monochromatic magnitudes of 0.03 for Vega.

This theoretically defined photometric system also provides transformations between the Johnson/Kron-Cousins system and the supernova system. We use theoretically derived transformations to convert the known $V, R$, and $I$ magnitudes of Landolt (1992) standard fields into B35, V35, B45, V45 photometry.

On nights that are photometric, we observe Landolt standard fields with the B35, V35, B45, V45 filters and measure the stars' instrumental magnitudes from apertures large enough to collect all the stellar light. We then derive the transformation from the supernova system to the instrumental system as a function of the instrumental magnitudes, supernova system colors, and observed air mass. Because our theoretical response functions are very similar to the instrumental response functions, our measured color coefficients were small, typically less than 0.02 mag per mag of B45 - V45 or B35 - V35. These long-wavelength filters also reduced the effect of atmospheric extinction (compared to $B$ and $V$ ). Typical extinction coefficients were $0.11,0.09$, 0.07, and 0.06 mag per air mass for B35, B45, V35, and V45, respectively.

Isolated stars on each supernova frame were selected as local standards. The magnitudes of the local standards were determined from the transformation of their instrumental magnitudes, measured from similarly large apertures. The final transformed magnitudes of these local standards, averaged over three photometric nights, are given in Table 3. The locations of the local standards and the $\mathrm{SNe}$ are shown in Figure 2. The uncertainties in the local standards' magnitudes are the quadrature sum of the uncertainty (dispersion) of the instrumental transformations (typically $0.02 \mathrm{mag}$ ) and the individual uncertainties from photon (Poisson) statistics. The dispersion in the instrumental transformation quantifies the errors due to imperfect flat-fielding, small changes in the atmospheric transparency, incomplete empirical modeling of the response function, and seeing variations. This uncertainty is valid for any single observation of the local standards.

To measure the brightness of the supernovae free from host galaxy contamination, we obtained deep images of the hosts a year after, or months before, the discovery of the $\mathrm{SNe}$. These images were used to subtract digitally a host's light from the supernova's light, leaving only the stellar point-spread function (PSF). The algorithms employed to match the resolution, intensity, and coordinate frames of images prior to subtraction are described in Schmidt et al. (1998). The brightness of the SNe in these uncrowded fields was then measured relative to the calibrated local standard stars in the field by fitting a model of a PSF to the stars and supernova using the DoPHOT algorithm (Schmidt et al. 1998; Mateo \& Schechter 1989; Schechter, Mateo, \& Saha 1993). 
TABLE 3

SN Ia Field Local Standard Stars

\begin{tabular}{|c|c|c|c|c|}
\hline Star & B45 & V45 & B35 & V35 \\
\hline \multicolumn{5}{|c|}{ SN 1996E } \\
\hline 1. & $20.84(0.02)$ & $20.71(0.02)$ & $\ldots$ & $\ldots$ \\
\hline & $20.07(0.03)$ & $18.69(0.03)$ & $\ldots$ & $\ldots$ \\
\hline & $19.60(0.03)$ & $19.22(0.03)$ & $\ldots$ & $\ldots$ \\
\hline $4 \ldots \ldots$ & $19.76(0.03)$ & $18.35(0.03)$ & $\ldots$ & $\ldots$ \\
\hline $5 \ldots \ldots$ & 19.16(0.03) & $18.29(0.03)$ & $\ldots$ & $\ldots$ \\
\hline & $20.85(0.02)$ & $20.52(0.02)$ & $\cdots$ & $\cdots$ \\
\hline \multicolumn{5}{|c|}{ SN 1996H } \\
\hline 1. & $18.16(0.02)$ & $17.84(0.02)$ & $\ldots$ & $\ldots$ \\
\hline 2. & $19.96(0.02)$ & $18.50(0.02)$ & $\ldots$ & $\ldots$ \\
\hline & $21.13(0.02)$ & $19.41(0.02)$ & $\ldots$ & $\ldots$ \\
\hline $4 \ldots \ldots$ & $20.76(0.02)$ & $19.21(0.02)$ & $\ldots$ & $\ldots$ \\
\hline & $19.62(0.02)$ & $19.23(0.02)$ & $\ldots$ & $\ldots$ \\
\hline 6. & $20.02(0.02)$ & $19.69(0.02)$ & $\cdots$ & $\cdots$ \\
\hline \multicolumn{5}{|c|}{ SN 1996I } \\
\hline $1 .$. & $19.59(0.02)$ & $18.67(0.02)$ & $\ldots$ & $\ldots$ \\
\hline $2 \ldots \ldots$ & $22.35(0.02)$ & $20.72(0.02)$ & $\ldots$ & $\ldots$ \\
\hline $3 \ldots \ldots$ & $20.62(0.02)$ & 18.93(0.02) & $\ldots$ & $\ldots$ \\
\hline $4 \ldots \ldots$ & $20.22(0.02)$ & 18.97(0.02) & $\ldots$ & $\ldots$ \\
\hline $5 \ldots \ldots$ & $17.46(0.02)$ & $17.18(0.02)$ & $\ldots$ & $\ldots$ \\
\hline & $18.02(0.02)$ & $17.55(0.02)$ & $\cdots$ & $\ldots$ \\
\hline \multicolumn{5}{|c|}{ SN 1996J } \\
\hline 1. & $18.59(0.02)$ & $17.38(0.02)$ & $19.09(0.02)$ & $17.85(0.02)$ \\
\hline 2. & $20.27(0.02)$ & $19.49(0.02)$ & $20.74(0.02)$ & $19.78(0.02)$ \\
\hline $3 \ldots \ldots$ & $20.20(0.02)$ & $19.45(0.02)$ & $20.70(0.02)$ & $19.79(0.02)$ \\
\hline $4 \ldots \ldots$ & $19.63(0.02)$ & $18.67(0.02)$ & $20.09(0.02)$ & $19.06(0.02)$ \\
\hline & $21.12(0.02)$ & $19.63(0.02)$ & $21.69(0.02)$ & $20.20(0.02)$ \\
\hline $6 \ldots \ldots$ & $20.27(0.02)$ & $20.00(0.02)$ & $20.57(0.02)$ & $20.06(0.02)$ \\
\hline \multicolumn{5}{|c|}{ SN 1996K } \\
\hline 1. & $19.06(0.02)$ & $18.81(0.02)$ & $19.22(0.02)$ & $18.88(0.02)$ \\
\hline $2 \ldots \ldots$ & $19.76(0.03)$ & $19.43(0.03)$ & $19.94(0.03)$ & $19.53(0.03)$ \\
\hline $3 \ldots \ldots$ & $19.41(0.03)$ & $18.17(0.02)$ & $19.90(0.03)$ & $18.62(0.02)$ \\
\hline $4 \ldots \ldots$ & $19.84(0.03)$ & $18.64(0.02)$ & $20.28(0.03)$ & $19.06(0.02)$ \\
\hline $5 \ldots \ldots$ & $19.30(0.02)$ & $17.70(0.02)$ & $19.84(0.02)$ & $18.25(0.02)$ \\
\hline & $19.04(0.02)$ & $18.06(0.02)$ & $19.45(0.02)$ & $18.40(0.02)$ \\
\hline $7 \ldots \ldots$ & $18.05(0.02)$ & $17.17(0.02)$ & $18.47(0.02)$ & $17.49(0.02)$ \\
\hline \multicolumn{5}{|c|}{ SN 1996R } \\
\hline $1 \ldots \ldots$ & $17.29(0.03)$ & $16.61(0.02)$ & $18.01^{\mathrm{V}}(0.03)$ & $\cdots$ \\
\hline $2 \ldots \ldots$ & $18.15(0.03)$ & $17.78(0.03)$ & $18.48^{\mathrm{V}}(0.03)$ & $\ldots$ \\
\hline $3 \ldots \ldots$ & $19.05(0.03)$ & $18.67(0.03)$ & $19.51^{\mathrm{V}}(0.03)$ & $\ldots$ \\
\hline $4 \ldots \ldots$ & $19.20(0.03)$ & $18.22(0.03)$ & $20.02^{\mathrm{V}}(0.03)$ & $\ldots$ \\
\hline $5 \ldots \ldots$ & $18.06(0.03)$ & $17.64(0.03)$ & $18.54^{\mathrm{V}}(0.03)$ & $\cdots$ \\
\hline \multicolumn{5}{|c|}{ SN 1996T } \\
\hline $1 \ldots \ldots$ & $18.29(0.02)^{\mathrm{V}}$ & $18.01(0.02)^{\mathrm{R}}$ & $\cdots$ & $\cdots$ \\
\hline $2 \ldots \ldots$ & $19.77(0.02)^{\mathrm{V}}$ & $18.57(0.02)^{\mathrm{R}}$ & $\ldots$ & $\ldots$ \\
\hline $3 \ldots \ldots$ & $20.43(0.02)^{\mathrm{V}}$ & $19.31(0.02)^{\mathrm{R}}$ & $\ldots$ & $\ldots$ \\
\hline $4 \ldots \ldots$ & $21.28(0.02)^{\mathrm{V}}$ & $20.57(0.02)^{\mathrm{R}}$ & $\ldots$ & $\ldots$ \\
\hline & $21.28(0.02)^{\mathrm{V}}$ & $20.27(0.02)^{\mathrm{R}}$ & $\ldots$ & $\cdots$ \\
\hline & $21.34(0.02)^{\mathrm{V}}$ & $20.37(0.02)^{R}$ & $\cdots$ & $\cdots$ \\
\hline \multicolumn{5}{|c|}{ SN 1995ao } \\
\hline $1 \ldots \ldots$ & $20.36(0.03)$ & $20.15(0.03)$ & $20.59(0.03)$ & $20.19(0.03)$ \\
\hline $2 \ldots \ldots$ & $\ldots$ & $\ldots$ & $17.89(0.03)$ & $17.50(0.03)$ \\
\hline $3 \ldots \ldots$ & $20.09(0.03)$ & $19.50(0.03)$ & $20.50(0.03)$ & $19.79(0.03)$ \\
\hline $4 \ldots \ldots$ & $20.10(0.03)$ & $19.75(0.03)$ & $20.39(0.03)$ & $19.86(0.03)$ \\
\hline $5 \ldots \ldots$ & $16.37(0.03)$ & $15.47(0.03)$ & $16.62(0.03)$ & $15.73(0.03)$ \\
\hline $6 \ldots \ldots$ & $\ldots$ & $\ldots$ & $17.32(0.03)$ & $16.81(0.03)$ \\
\hline
\end{tabular}

TABLE 3-Continued

\begin{tabular}{lcccc}
\hline \hline Star & B45 & V45 & B35 & V35 \\
\hline \multicolumn{5}{c}{ SN 1995ap } \\
\hline $1 \ldots \ldots$ & $19.49(0.03)$ & $18.21(0.03)$ & $20.28(0.03)$ & $18.69(0.02)$ \\
$2 \ldots \ldots$ & $19.19(0.03)$ & $18.76(0.03)$ & $19.54(0.03)$ & $18.88(0.02)$ \\
$3 \ldots \ldots$ & $18.97(0.03)$ & $18.24(0.03)$ & $19.43(0.03)$ & $18.47(0.02)$ \\
$4 \ldots \ldots$ & $19.67(0.03)$ & $18.61(0.03)$ & $20.31(0.02)$ & $18.98(0.02)$ \\
$5 \ldots \ldots$ & $20.51(0.03)$ & $19.44(0.03)$ & $21.16(0.02)$ & $19.81(0.02)$ \\
$6 \ldots \ldots$ & $20.90(0.03)$ & $20.31(0.03)$ & $21.53(0.02)$ & $20.50(0.02)$ \\
\hline
\end{tabular}

NoTE.-Uncertainties in magnitudes are listed in parentheses.

Systematic and statistical components of error were evaluated by measuring the brightness of artificial stars added to the subtracted frames. These artificial stars had the same brightness and background as the measured $\mathrm{SNe}$ (Schmidt et al. 1998). The "systematic" error was measured from the difference in the mean magnitude of the artificial stars before and after the image processing (i.e., alignment, scaling, "blurring," and subtracting). The systematic errors were always less than $0.1 \mathrm{mag}$ and were of either sign. Any significant systematic error is likely the result of a mismatch in the global properties of the template image and SN image based on only examining a local region of the two images. A correction based on the systematic error determined from the artificial stars was applied to the measured SN magnitude to yield an unbiased estimate of the SN magnitude. The dispersion of the recovered artificial magnitudes about their mean was assigned to the statistical uncertainty of the SN magnitude.

The supernova PSF magnitudes were transformed to the B35, V35, B45, V45 system using the local standard magnitudes and the color coefficients derived from observations of the Landolt standards. The final SN light curves are the average of the results derived from five or six local standards, weighted by the uncertainty of each local standard star. The light curves are listed in Table 4 and displayed in Figure 3. The SN magnitude errors are derived from the artificial star measurements as described above.

The small color and atmospheric extinction coefficients give us confidence that the supernova photometry accurately transformed to the $\mathrm{B} 35, \mathrm{~V} 35, \mathrm{~B} 45, \mathrm{~V} 45$ system. However, it is well known that a nonstellar flux distribution can produce substantial systematic errors in supernova photometry (Menzies 1989). We have anticipated this problem by using identical filter sets at the various observatories and by defining our photometric system with actual instrumental response functions. To measure the size of this effect on our SN photometry, we have calculated the systematic error incurred from the differences in the instrumental response functions of different observatories we employed. Spectrophotometric calculations from SN Ia spectra using various instrumental response functions show that the expected differences are less than $0.01 \mathrm{mag}$ and can safely be ignored.

\section{ANALYSIS}

\subsection{K-Corrections}

A strong empirical understanding of SN Ia light curves has been garnered from intensive monitoring of SNe Ia at $z \leq 0.1$ through $B$ and $V$ passbands (Hamuy et al. 1996a; Riess 1996; Riess et al. 1998c; Ford et al. 1993; Branch 1998 and references therein). We use this understanding to 


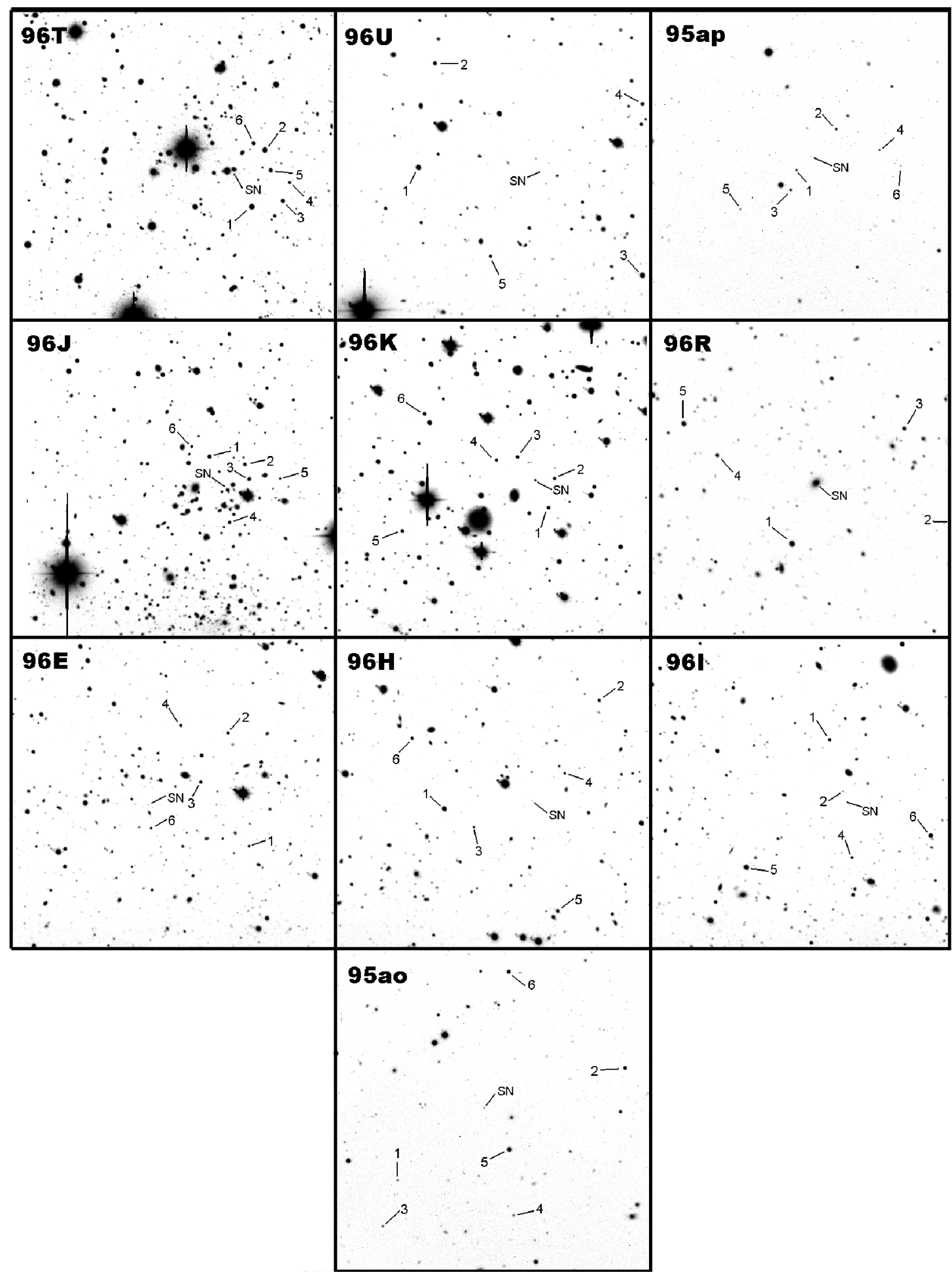

FIG. 2.-Local standard stars in the fields of SNe Ia. The stars are listed in Table 2, and the locations of the stars and SNe are indicated in the figure. The orientation of each field is east to the right and north at the top. The width and length of each field is $96 \mathrm{E}=4^{\prime} .9,96 \mathrm{H}=4^{\prime} .9,96 \mathrm{I}=4^{\prime} .9,96 \mathrm{~J}=4^{\prime} .9,96 \mathrm{~K}=4^{\prime} .9$, $96 \mathrm{R}=5^{\prime} .0,96 \mathrm{~T}=4^{\prime} .9,96 \mathrm{U}=4^{\prime} .9,95 \mathrm{ao}=4^{\prime} .8,95 \mathrm{ap}=4^{\prime} .8$.

compare the light curves of the high-redshift and lowredshift samples at the same rest wavelength. By a judicious choice of filters, we minimize the differences between $B$ and $V$ rest-frame light observed for distant $\mathrm{SNe}$ and their nearby counterparts. Nevertheless, the range of redshifts involved makes it difficult to eliminate all such differences. We therefore employ " $K$-corrections" to convert the observed magnitudes to rest-frame $B$ and $V$ (Oke \&
Sandage 1968; Hamuy et al. 1993b; Kim, Goobar, \& Perlmutter 1996; Schmidt et al. 1998).

The cross-band $K$-correction for $\mathrm{SNe}$ Ia has been described as a function of the observed and rest-frame filter transmissions, the redshift of the supernova, and the age of the supernova (see eq. [1] of Kim et al. 1996). Such a $K$-correction assumes that the spectral energy distribution of all SNe Ia of a given age is homogeneous, yet it has been 
TABLE 4

Type Ia SuPERnova Light Curves Corrected TO THE REST FraME

\begin{tabular}{ccccc}
\hline \hline \multicolumn{1}{c}{ JD $^{\mathrm{a}}$} & $B$ & $V$ & $K_{B}$ & $K_{V}$ \\
\hline \multicolumn{5}{c}{ SN 1996E } \\
\hline $127.6 \ldots \ldots$ & $23.04(0.09)$ & $\ldots$ & -0.74 & $\ldots$ \\
$128.6 \ldots \ldots$ & $23.02(0.05)$ & $22.72(0.08)$ & -0.74 & -0.86 \\
$132.1 \ldots \ldots$ & $23.23(0.11)$ & $\ldots$ & -0.77 & $\ldots$ \\
$134.6 \ldots \ldots$ & $23.39(0.10)$ & $22.84(0.26)$ & -0.73 & -0.85 \\
$135.5 \ldots \ldots$ & $23.41(0.13)$ & $22.95(0.06)$ & -0.74 & -0.85 \\
$138.7 \ldots \ldots$ & $23.80(0.12)$ & $23.14(0.15)$ & -0.76 & -0.84 \\
$139.6 \ldots \ldots$ & $23.65(0.15)$ & $23.57(0.33)$ & -0.76 & -0.84 \\
$157.6 \ldots \ldots$ & $25.14(0.18)$ & $24.42(0.77)$ & -0.82 & -0.91 \\
$163.7 \ldots \ldots$. & $\ldots$ & $23.78(0.50)$ & $\ldots$ & -0.91
\end{tabular}

\begin{tabular}{ccccc}
$163.7 \ldots \ldots$ & $\cdots$ & $23.78(0.50)$ & $\cdots$ & -0.91 \\
\hline \multicolumn{5}{c}{ SN $1996 \mathrm{H}$} \\
\hline $127.6 \ldots \ldots$ & $23.32(0.13)$ & $\ldots$ & -0.54 & $\ldots$ \\
$128.6 \ldots \ldots$ & $23.39(0.09)$ & $23.42(0.14)$ & -0.58 & -1.17 \\
$132.1 \ldots \ldots$ & $23.27(0.30)$ & $23.56(0.37)$ & -0.56 & -1.15 \\
$134.6 \ldots \ldots$ & $23.48(0.11)$ & $23.58(0.19)$ & -0.63 & -1.10 \\
$135.5 \ldots \ldots$ & $23.29(0.18)$ & $23.40(0.10)$ & -0.47 & -1.12 \\
$136.6 \ldots \ldots$ & $23.29(0.14)$ & $\ldots$ & -0.45 & $\ldots$ \\
$138.7 \ldots \ldots$ & $23.47(0.10)$ & $23.64(0.15)$ & -0.62 & -1.06 \\
$139.6 \ldots \ldots$ & $23.55(0.18)$ & $23.56(0.25)$ & -0.67 & -1.04 \\
$140.6 \ldots \ldots$ & $23.58(0.18)$ & $24.13(0.11)$ & -0.62 & -1.03 \\
$141.6 \ldots \ldots$ & $23.62(0.12)$ & $\ldots$ & -0.56 & $\ldots$ \\
$142.6 \ldots \ldots$ & $23.74(0.21)$ & $23.72(0.16)$ & -0.53 & -1.04 \\
$157.6 \ldots \ldots$ & $24.44(0.22)$ & $23.86(0.28)$ & -0.47 & -0.69 \\
$161.6 \ldots \ldots$ & $24.60(0.22)$ & $\ldots$ & -0.44 & $\ldots$ \\
$164.6 \ldots \ldots$ & $\ldots$ & $24.62(0.30)$ & $\ldots$ & -0.61 \\
\hline \multicolumn{5}{c}{ SN 1996I }
\end{tabular}

\begin{tabular}{lcccc}
\multicolumn{5}{c}{ SN 1996I } \\
\hline $128.6 \ldots \ldots$ & $23.45(0.08)$ & $\ldots$ & -0.68 & $\ldots$ \\
$132.1 \ldots \ldots$ & $23.62(0.22)$ & $23.25(0.22)$ & -0.67 & -0.96 \\
$134.6 \ldots \ldots$ & $23.57(0.06)$ & $23.66(0.15)$ & -0.62 & -1.02 \\
$135.5 \ldots \ldots$ & $23.54(0.06)$ & $23.65(0.20)$ & -0.62 & -1.01 \\
$136.6 \ldots \ldots$ & $23.58(0.10)$ & $23.74(0.28)$ & -0.70 & -1.00 \\
$138.7 \ldots \ldots$ & $23.81(0.14)$ & $23.82(0.17)$ & -0.69 & -0.97 \\
$140.6 \ldots \ldots$ & $24.28(0.36)$ & $23.66(0.36)$ & -0.64 & -1.00 \\
$142.6 \ldots \ldots$ & $24.13(0.10)$ & $24.02(0.22)$ & -0.65 & -0.97 \\
$157.6 \ldots \ldots$ & $25.38(0.17)$ & $24.39(0.30)$ & -0.55 & -0.73 \\
$161.6 \ldots \ldots$ & $25.25(0.31)$ & $\ldots$ & -0.55 & $\ldots$
\end{tabular}

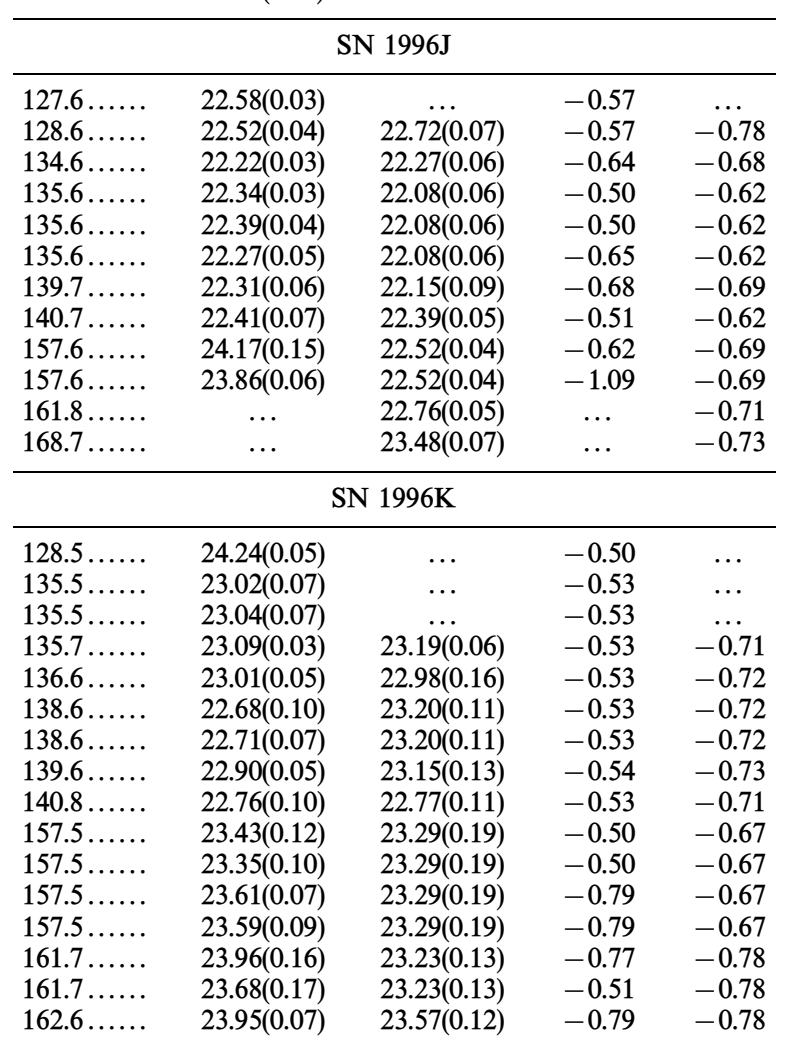

TABLE 4-Continued

\begin{tabular}{|c|c|c|c|c|}
\hline $\mathrm{JD}^{\mathrm{a}}$ & $B$ & V & $K_{B}$ & $K_{V}$ \\
\hline $165.6 \ldots \ldots$ & $24.09(0.16)$ & $23.80(0.14)$ & -0.50 & -0.62 \\
\hline $168.5 \ldots \ldots$ & & $23.73(0.19)$ & $\ldots$ & -0.63 \\
\hline $169.6 \ldots \ldots$ & $24.92(0.25)$ & $\ldots$ & -0.50 & $\ldots$ \\
\hline 169.7 . & $24.91(0.26)$ & ... & -0.86 & $\ldots$ \\
\hline \multicolumn{5}{|c|}{ SN 1996R } \\
\hline 157.7. & & $20.81(0.02)$ & $\cdots$ & -0.33 \\
\hline $158.7 \ldots \ldots$ & $\ldots$ & $20.92(0.03)$ & & -0.33 \\
\hline 167.7. & $22.24(0.03)$ & & -0.63 & $\ldots$ \\
\hline $191.7 \ldots \ldots$ & $\ldots$ & $22.76(0.09)$ & $\ldots$ & -0.35 \\
\hline \multicolumn{5}{|c|}{ SN 1996T } \\
\hline $161.7 \ldots \ldots$ & $21.24(0.02)$ & $21.27(0.03)$ & -0.38 & -0.44 \\
\hline $167.6 \ldots \ldots$ & $21.34(0.03)$ & $21.35(0.04)$ & -0.38 & -0.40 \\
\hline $191.7 \ldots \ldots$ & $22.73(0.20)$ & & -0.37 & $\cdots$ \\
\hline $212.6 \ldots \ldots$ & $23.35(0.35)$ & $22.81(0.09)$ & -0.36 & -0.29 \\
\hline \multicolumn{5}{|c|}{ SN 1996U } \\
\hline $158.7 .$. & $22.89(0.05)$ & & -0.73 & \\
\hline 160.7. & $22.73(0.11)$ & $22.88(0.18)$ & -0.73 & -0.85 \\
\hline 161.7. & $22.78(0.05)$ & $23.09(0.26)$ & -0.74 & -0.85 \\
\hline $165.7 \ldots \ldots$ & & $23.21(0.28)$ & & -0.86 \\
\hline $167.7 .$. & $22.94(0.10)$ & & -0.75 & $\ldots$ \\
\hline $186.7 \ldots \ldots$ & $24.23(0.17)$ & $23.49(0.28)$ & -0.90 & -0.86 \\
\hline $188.7 \ldots \ldots$ & $24.34(0.17)$ & $23.85(0.36)$ & -0.83 & -0.89 \\
\hline \multicolumn{5}{|c|}{ SN 1995ao } \\
\hline $46.6 \ldots \ldots$ & $21.85^{\mathrm{R}}(0.13)$ & & -0.75 & \\
\hline 51.6 . & $21.95(0.05)$ & $21.70(0.03)$ & -0.43 & -0.58 \\
\hline \multicolumn{5}{|c|}{ SN 1995ap } \\
\hline 39.6 . & & $22.85(0.14)$ & & -0.44 \\
\hline $46.6 \ldots \ldots$ & $21.96^{\mathrm{R}}(0.10)$ & $21.57(0.08)$ & -0.56 & -0.45 \\
\hline $48.6 \ldots \ldots$ & & $21.49(0.11)$ & .. & -0.45 \\
\hline $51.6 \ldots \ldots$ & $21.84(0.09)$ & $21.40(0.08)$ & -0.20 & -0.89 \\
\hline
\end{tabular}

NotE.-Uncertainties in magnitudes are listed in parentheses.

${ }^{a}$ Actually JD - 2,450,000.

shown (Pskovskii 1984; Phillips et al. 1987; Phillips 1993; Leibundgut et al. 1993; Nugent et al. 1995; Riess et al. 1996a; Phillips et al. 1998; Lira 1995; see Appendix of this paper) that at a given age, the colors of SNe Ia exhibit real variation related to the absolute magnitude of the supernova.

A variation in SN Ia color, at a fixed phase, could have dire consequences for determining accurate $K$-corrections. An appropriate $K$-correction quantifies the difference between the supernova light that falls into a standard passband (e.g., $B$ ) at $z=0$ and that which falls into the filters we employ to observe a redshifted SN Ia. Differences in SN Ia color, at a fixed phase, would alter the appropriate $K$-correction. We need to know the color of each supernova to determine its $K$-correction precisely. Differences in SN Ia color can arise from interstellar extinction or intrinsic properties of the supernova such as a variation in photospheric temperature (Nugent et al. 1995).

Nugent et al. (1998b) have shown that, to within 0.01 mag, the effects of both extinction and intrinsic variations on the SN Ia spectral energy distribution near rest-frame $B$ and $V$, and hence on the $K$-correction, can be reproduced by application of a Galactic reddening law (Cardelli, Clayton, \& Mathis 1989) to the spectra. The difference in color, at a given age, between an individual SN Ia and a fiducial SN Ia is quantified by a color excess, $E_{B-V}$, and determines the effects of either extinction or intrinsic varia- 

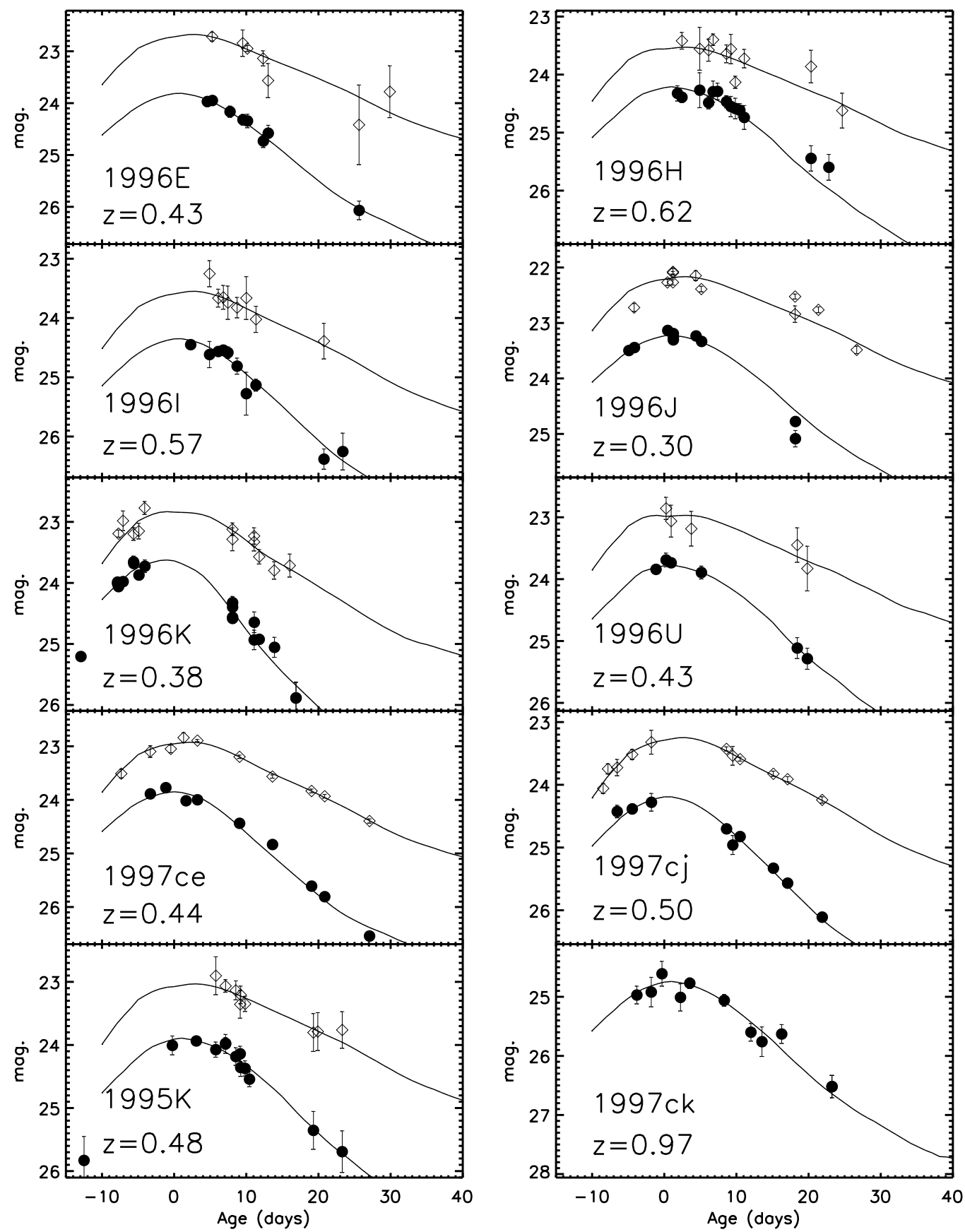

Fig. 3.-Light curves of high-redshift SNe Ia. $B$ (filled symbols) and $V$ (open symbols) photometry in the rest frame of 10 well-observed SNe Ia is shown with $B$ increased by $1 \mathrm{mag}$ for ease of view. The lines are the empirical MLCS model fits to the data. Supernova age is shown relative to $B$ maximum.

tion on the spectra and observed colors of the SNe. For most epochs, filter combinations, and redshifts, the variation of the $K$-correction with the observed variations of color excess is only 0.01 to $0.05 \mathrm{mag}$. For redshifts that poorly match the rest-frame wavelengths to the observed wavelengths, the custom $K$-correction for very red or very blue SNe Ia can differ from the standard $K$-correction by 0.1 to $0.2 \mathrm{mag}$.

This prescription requires the age and observed color for each observation to be known before its $K$-correction can be calculated. The age is best determined from fitting the light curve's time of maximum. Yet we must use the $K$ correction to determine the time of maximum and the true color of each epoch. This conundrum can be solved by iteratively converging to a solution by repeated cycles of
$K$-correcting and empirical fitting of the light curves. Table 4 lists the final cross-band $K$-corrections we used to convert the observations to the rest-frame passbands. We have also corrected the light curves in Figure 3 and our light curve fits for a $1+z$ time dilation, expected in an expanding universe (Riess et al. 1997).

\subsection{Light Curve Fitting}

As described in $\S 1$, empirical models for $\mathrm{SNe}$ Ia light curves that employ the observed correlation between light curve shape, luminosity, and color have led to significant improvements in the precision of distance estimates derived from SNe Ia (Hamuy et al. 1995, 1996c; Riess et al. 1995, 1996a; Tripp 1997, 1998). Here we employ the MLCS method prescribed by Riess et al. (1996a) as reanalyzed in 
the Appendix, and the template-fitting method of Hamuy et al. $(1995,1996 \mathrm{~d})$ to fit the light curves in Table 4.

The growing sample of well-observed SN Ia light curves (Hamuy et al. 1996a; Riess 1996; Riess et al. 1998c; Ford et al. 1993) justifies refinements in the MLCS method that are described in the Appendix. These include a new derivation of the relation between light curve shape, luminosity, and color from SNe Ia in the Hubble flow using redshift as the distance indicator. In addition, this empirical description has been extended to a second-order (i.e., quadratic) relation between SN Ia luminosity and light curve shape. A more realistic a priori probability distribution for extinction has been utilized from the calculations of Hatano, Branch, \& Deaton (1998). Further, we now quantify the residual correlations between observations of dissimilar time, passband, or both. The empirical model for a SN Ia light and color curve is still described by four parameters: a date of maximum $(t)$, a luminosity difference $(\Delta)$, an apparent distance $\left(\mu_{B}\right)$, and an extinction $\left(A_{B}\right)$. Because of the redshifts of the SN host galaxies, we first correct the supernova light curves for Galactic extinction (Burstein \& Heiles 1982) and then determine host galaxy extinction.

To treat the high- and low-redshift SNe Ia consistently, we restricted the MLCS fits to the nearby SNe Ia observations in $B$ and $V$ within 40 days after maximum brightness in the rest frame. This is the age by which all high-redshift light curve observations ended. Because of this restriction, we also limited our consideration of nearby $\mathrm{SNe}$ Ia to those with light curves which began no later than $\sim 5$ days after $B$ maximum. Although more precise distance estimates could be obtained for the nearby sample by including later data and additional colors, the nearby sample is large enough to determine the nearby expansion rate to sufficient precision. The parameters of the MLCS fits to $27 \mathrm{SNe}$ Ia in the nearby Hubble flow $(0.01<z<0.13$; Hamuy et al. 1996a; Riess et al. 1998c) are given in Table 10 below.

In Table 5 we list the parameters of the MLCS fits to six SN Ia light curves presented here (SNe 1996E, 1996H, 1996I, 1996J, 1996K, 1996U) and for three SNe Ia from our previous work (SNe 1995K, 1997ce, 1997cj; Garnavich et al. 1998a; Schmidt et al. 1998). We have placed all MLCS distances on the Cepheid distance scale using Cepheid distances to galaxies hosting photoelectrically observed $\mathrm{SNe}$ Ia: SN 1981B, SN 1990N, and SN 1972E (Saha et al. 1994, 1997; Riess et al. 1996a). However, conclusions about the values of the cosmological parameters $\Omega_{M}, \Omega_{\Lambda}$, and $q_{0}$ are independent of the distance scale.

An additional supernova, SN 1997ck, was studied by Garnavich et al. (1998a) in a galaxy with $z=0.97$. Its restframe $B$ light curve was measured with the HST (see Fig. 3).
Although this object lacks a spectroscopic classification and useful color information, its light curve shape and peak luminosity are consistent with those of a typical SN Ia. Owing to the uncertainty in this object's extinction and classification, we will analyze the $\mathrm{SNe}$ Ia distances both with and without this most distant object.

We have also determined the distances to the same 27 nearby $\mathrm{SNe}$ Ia and the 10 well-observed high-redshift events using a template-fitting approach (Hamuy et al. 1995, 1996d). The maximum-light magnitudes and the initial decline rate parameter $\Delta m_{15}(B)$ for a given SN Ia are derived by comparing the goodness of fits of the photometric data to a set of six template SN Ia light curves selected to cover the full range of observed decline rates. The intrinsic luminosity of the $\mathrm{SN}$ is then corrected to a standard value of the decline rate $\left[\Delta m_{15}(B)=1.1\right]$ using a linear relation between $\Delta m_{15}(B)$ and the luminosities for a set of SNe Ia in the Hubble flow (Phillips et al. 1998). An extinction correction has been applied to these distances based on the measured color excess at maximum light using the relation between $\Delta m_{15}(B)$ and the unreddened $\mathrm{SN}$ Ia color at maximum light (Phillips et al. 1998). These extinction measurements employ the same Bayesian filter (in the Appendix) used for the MLCS fits. The final distance moduli are also on the Cepheid distance scale as described by Hamuy et al. (1996c) and Phillips et al. (1998). Parameters of these fits to the nearby and high-redshift SNe Ia are provided in Table 10 and Table 6, respectively.

For both the MLCS and template-fitting methods, the fit to the data determines the light curve parameters and their uncertainties. The "goodness" of the fits was within the expected statistical range with the exception of SN 1996J. This supernova is at a measured redshift of $z=0.30$, but some of the observations were obtained through a set of filters optimized for $z=0.45$. The uncertainty from this mismatch and the additional uncertainty from separate calibrations of the local standards' magnitudes in two sets of filters may be the source of the poor result for this object.

Four remaining $\mathrm{SNe}$ Ia presented here (SNe 1995ao, 1995ap, 1996T, and 1996R) are too sparsely sampled to provide meaningful light curves fitted by either of the light curve fitting methods. However, Riess et al. (1998b) describe a technique to measure the distance to sparsely observed SNe that lack well-sampled light curves. This "snapshot" method measures the age and the luminosity/light curve shape parameter from a SN Ia spectrum using techniques from Riess et al. (1997) and Nugent et al. (1995). An additional photometric epoch in two passbands (with host galaxy templates if needed) provides enough information to determine the extinction-free distance. For the four sparsely

TABLE 5

High-z MLCS SN Ia Light Curve Parameters

\begin{tabular}{ccccccc}
\hline \hline SN & $z$ & $m_{B}^{\max }$ & $m_{V}^{\max }$ & $\Delta$ & $A_{B}$ & $\mu_{0}\left(\sigma_{\mu_{0}}\right)$ \\
\hline $1996 \mathrm{E} \ldots \ldots$ & 0.43 & $22.81(0.21)$ & $22.72(0.23)$ & $-0.08(0.19)$ & 0.31 & $41.74(0.28)$ \\
$1996 \mathrm{H} \ldots \ldots$. & 0.62 & $23.23(0.19)$ & $23.56(0.18)$ & $-0.42(0.16)$ & 0.00 & $42.98(0.17)$ \\
$1996 \mathrm{I} \ldots \ldots$. & 0.57 & $23.35(0.28)$ & $23.59(0.26)$ & $-0.06(0.26)$ & 0.00 & $42.76(0.19)$ \\
$1996 \mathrm{~J} \ldots \ldots$. & 0.30 & $22.23(0.12)$ & $22.21(0.11)$ & $-0.22(0.10)$ & 0.24 & $41.38(0.24)$ \\
$1996 \mathrm{~K} \ldots \ldots$. & 0.38 & $22.64(0.12)$ & $22.84(0.14)$ & $0.29(0.06)$ & 0.00 & $41.63(0.20)$ \\
$1996 \mathrm{U} \ldots \ldots$. & 0.43 & $22.78(0.22)$ & $22.98(0.30)$ & $-0.52(0.29)$ & 0.00 & $42.55(0.25)$ \\
$1997 \mathrm{ce} \ldots \ldots$. & 0.44 & $22.85(0.09)$ & $22.95(0.09)$ & $0.07(0.08)$ & 0.00 & $41.95(0.17)$ \\
$1997 \mathrm{cj} \ldots \ldots$. & 0.50 & $23.19(0.11)$ & $23.29(0.12)$ & $-0.04(0.11)$ & 0.00 & $42.40(0.17)$ \\
$1997 \mathrm{ck} \ldots \ldots$. & 0.97 & $24.78(0.25)$ & $\ldots$ & $-0.19(0.23)$ & $\ldots$ & $44.39(0.30)$ \\
$1995 \mathrm{~K} \ldots \ldots$. & 0.48 & $22.91(0.13)$ & $23.08(0.20)$ & $-0.33(0.26)$ & 0.00 & $42.45(0.17)$ \\
\hline
\end{tabular}

NoTE.-Uncertainties in magnitudes are listed in parentheses. 
TABLE 6

High- $z$ Template-Fitting SN Ia Light CuRve Parameters

\begin{tabular}{ccccccc}
\hline \hline SN & $z$ & $m_{B}^{\max }$ & $m_{V}^{\max }$ & $\Delta M_{15}(B)$ & $A_{B}$ & $\mu_{0}\left(\sigma_{\mu_{0}}\right)$ \\
\hline $1996 \mathrm{E} \ldots \ldots$. & 0.43 & $22.72(0.19)$ & $22.60(0.12)$ & $1.18(0.13)$ & 0.10 & $42.03(0.22)$ \\
$1996 \mathrm{H} \ldots \ldots$. & 0.62 & $23.31(0.06)$ & $23.57(0.06)$ & $0.87(0.05)$ & 0.00 & $43.01(0.15)$ \\
$1996 \mathrm{I} \ldots \ldots$. & 0.57 & $23.42(0.08)$ & $23.61(0.08)$ & $1.39(0.17)$ & 0.00 & $42.83(0.21)$ \\
$1996 \mathrm{~J} \ldots \ldots$. & 0.30 & $22.28(0.05)$ & $22.06(0.05)$ & $1.27(0.27)$ & 0.64 & $40.99(0.25)$ \\
$1996 \mathrm{~K} \ldots \ldots$. & 0.38 & $22.80(0.05)$ & $22.86(0.08)$ & $1.31(0.14)$ & 0.00 & $42.21(0.18)$ \\
$1996 \mathrm{U} \ldots \ldots$. & 0.43 & $22.77(0.05)$ & $22.96(0.11)$ & $1.18(0.10)$ & 0.00 & $42.34(0.17)$ \\
$1997 \mathrm{ce} \ldots \ldots$. & 0.44 & $22.83(0.05)$ & $22.92(0.05)$ & $1.30(0.06)$ & 0.00 & $42.26(0.16)$ \\
$1997 \mathrm{cj} \ldots \ldots$. & 0.50 & $23.29(0.05)$ & $23.29(0.05)$ & $1.16(0.03)$ & 0.09 & $42.70(0.16)$ \\
$1997 \mathrm{ck} \ldots \ldots$. & 0.97 & $24.78(0.16)$ & $\ldots$. & $1.00(0.17)$ & $\ldots$ & $44.30(0.19)$ \\
$1995 \mathrm{~K} \ldots \ldots$. & 0.48 & $22.92(0.08)$ & $23.07(0.07)$ & $1.16(0.18)$ & 0.00 & $42.49(0.17)$ \\
\hline
\end{tabular}

NoTE.-Uncertainties in magnitudes are listed in parentheses.

observed SNe Ia in our sample, we have measured the SN parameters with this method and list them in Table 7. This sample of sparsely observed, high-redshift SNe Ia is augmented by distances for SN 1997I $(z=0.17)$ and SN 1997ap $(z=0.83)$ given by Riess et al. (1998b).

For all SN Ia distance measurements, the dominant source of statistical uncertainty is the extinction measurement. The precision of our determination of the true extinction is improved using our prior understanding of its magnitude and direction (Riess et al. 1996a; see Appendix).

\section{COSMOLOGICAL IMPLICATIONS OF TYPE Ia SUPERNOVAE}

\subsection{Cosmological Parameters}

Distance estimates from SN Ia light curves are derived from the luminosity distance,

$$
D_{L}=\left(\frac{\mathscr{L}}{4 \pi \mathscr{F}}\right)^{1 / 2}
$$

where $\mathscr{L}$ and $\mathscr{F}$ are the SN's intrinsic luminosity and observed flux, respectively. In Friedmann-RobertsonWalker cosmologies, the luminosity distance at a given redshift, $z$, is a function of the cosmological parameters. Limiting our consideration of these parameters to the Hubble constant, $H_{0}$, the mass density, $\Omega_{M}$, and the vacuum energy density (i.e., the cosmological constant), $\Omega_{\Lambda}$ (but see Caldwell, Dave, \& Steinhardt 1998; Garnavich et al. 1998a, $1998 \mathrm{~b}$ for other energy densities), the luminosity distance is

$$
\begin{aligned}
D_{L}= & c H_{0}^{-1}(1+z)\left|\Omega_{k}\right|^{-1 / 2} \sin n\left\{\left|\Omega_{k}\right|^{1 / 2}\right. \\
& \left.\times \int_{0}^{z} d z\left[(1+z)^{2}\left(1+\Omega_{M} z\right)-z(2+z) \Omega_{\Lambda}\right]^{-1 / 2}\right\},
\end{aligned}
$$

where $\Omega_{k}=1-\Omega_{M}-\Omega_{\Lambda}$ and $\sin n$ is $\sinh$ for $\Omega_{k} \geq 0$ and $\sin$ for $\Omega_{k} \leq 0$ (Carroll et al. 1992). For $D_{L}$ in units of mega-

TABLE 7

High-z SN Ia SNAPShOt PARAMETERS

\begin{tabular}{ccrccc}
\hline \hline SN & $z$ & \multicolumn{1}{c}{$t_{\text {spec }}$} & \multicolumn{1}{c}{$\Delta$} & $A_{V}$ & $\mu_{0}\left(\sigma_{\mu_{0}}\right)$ \\
\hline 1995ao $\ldots \ldots .$. & 0.30 & -2.8 & 0.35 & 0.00 & $40.74(0.60)$ \\
1995ap $\ldots \ldots .$. & 0.23 & -2.9 & 0.69 & 0.00 & $40.33(0.46)$ \\
1996R $\ldots \ldots .$. & 0.16 & 8.6 & 0.28 & 0.10 & $39.08(0.40)$ \\
1996T $\ldots \ldots .$. & 0.24 & -4.5 & -0.12 & 0.10 & $40.68(0.43)$ \\
$1997 \mathrm{I}^{\mathrm{a}} \ldots \ldots$. & 0.17 & 0.1 & -0.39 & 0.00 & $39.95(0.24)$ \\
1997ap ${ }^{\mathrm{a}} \ldots \ldots$ & 0.83 & -2.0 & 0.00 & 0.00 & $43.67(0.35)$ \\
\hline
\end{tabular}

${ }^{a}$ See Perlmutter et al. 1998; Riess et al. 1998b. parsecs, the predicted distance modulus is

$$
\mu_{p}=5 \log D_{L}+25 \text {. }
$$

Using the data described in $\S 2$ and the fitting methods of $\S 3$, we have derived a set of distances, $\mu_{0}$, for $\mathrm{SNe}$ with $0.01 \leq z \leq 0.97$. The available set of high-redshift $\mathrm{SNe}$ includes nine well-observed SNe Ia, six sparsely observed SNe Ia, and SN 1997ck $(z=0.97)$, whose light curve was well observed but lacks spectroscopic classification and color measurements. The Hubble diagrams for the nine well-observed SNe Ia plus SN 1997ck, with light curve distances calculated from the MLCS method and the template approach, are shown in Figures 4 and 5. The likelihood for the cosmological parameters can be determined from a $\chi^{2}$ statistic, where

$$
\chi^{2}\left(H_{0}, \Omega_{M}, \Omega_{\Lambda}\right)=\sum_{i} \frac{\left[\mu_{p, i}\left(z_{i} ; H_{0}, \Omega_{m}, \Omega_{\Lambda}\right)-\mu_{0, i}\right]^{2}}{\sigma_{\mu_{0, i}}^{2}+\sigma_{v}^{2}}
$$

and $\sigma_{v}$ is the dispersion in galaxy redshift (in units of distance moduli) due to peculiar velocities. This term also includes the uncertainty in galaxy redshift. We have calculated this $\chi^{2}$ statistic for a wide range of the parameters $H_{0}$, $\Omega_{M}$, and $\Omega_{\Lambda}$. We do not consider the unphysical region of parameter space where $\Omega_{M}<0$; equation (2) describes the effect of massive particles on the luminosity distance. There is no reason to expect that the evaluation of equation (2) for $\Omega_{M}<0$ has any correspondence to physical reality. We also neglect the region of $\left(\Omega_{M}, \Omega_{\Lambda}\right)$ parameter space, which gives rise to so-called "bouncing" or rebounding universes that do not monotonically expand from a "big bang" and for which equation (2) is not solvable (see Figs. 6 and 7) (Carroll et al. 1992).

Because of the large redshifts of our distant sample and the abundance of objects in the nearby sample, our analysis is insensitive to $\sigma_{v}$ within its likely range of $100 \mathrm{~km} \mathrm{~s}^{-1} \leq$ $\sigma_{v} \leq 400 \mathrm{~km} \mathrm{~s}^{-1}$ (Marzke et al. 1995; Lin et al. 1996). For our analysis we adopt $\sigma_{v}=200 \mathrm{~km} \mathrm{~s}^{-1}$. For high-redshift SNe Ia whose redshifts were determined from the broad features in the SN spectrum (see Table 1), we add $2500 \mathrm{~km}$ $\mathrm{s}^{-1}$ in quadrature to $\sigma_{v}$.

Separating the effects of matter density and vacuum energy density on the observed redshift-distance relation could in principle be accomplished with measurements of SNe Ia over a significant range of high redshifts (Goobar \& Perlmutter 1995). Because the matter density decreases with time in an expanding universe, while the vacuum energy density remains constant, the relative influence of $\Omega_{M}$ to $\Omega_{\Lambda}$ on the redshift-distance relation is a function of redshift. 


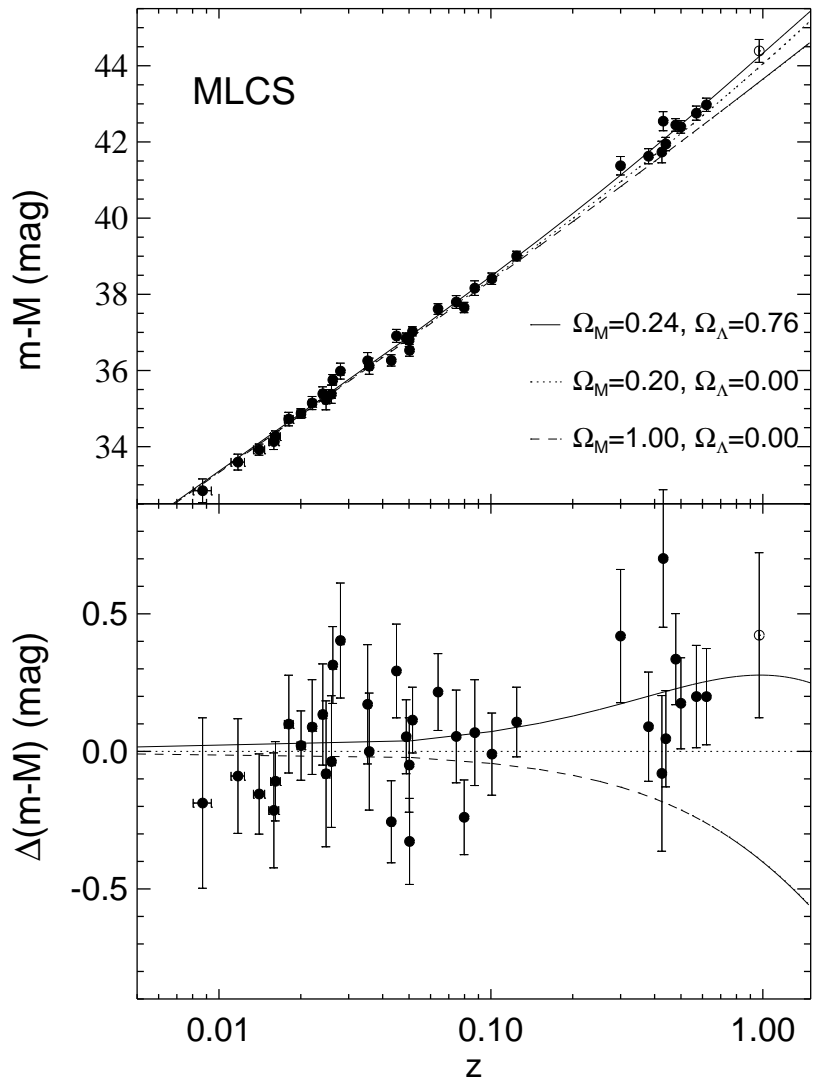

Fig. 4.-MLCS SNe Ia Hubble diagram. The upper panel shows the Hubble diagram for the low-redshift and high-redshift SNe Ia samples with distances measured from the MLCS method (Riess et al. 1995, 1996a; Appendix of this paper). Overplotted are three cosmologies: "low" and "high" $\Omega_{M}$ with $\Omega_{\Lambda}=0$ and the best fit for a flat cosmology, $\Omega_{M}=0.24$, $\Omega_{\Lambda}=0.76$. The bottom panel shows the difference between data and models with $\Omega_{M}=0.20, \Omega_{\Lambda}=0$. The open symbol is SN $1997 \mathrm{ck}(z=0.97)$, which lacks spectroscopic classification and a color measurement. The average difference between the data and the $\Omega_{M}=0.20, \Omega_{\Lambda}=0$ prediction is $0.25 \mathrm{mag}$.

The present data set has only a modest range of redshifts, so we can only constrain specific cosmological models or regions of $\left(\Omega_{M}, \Omega_{\Lambda}\right)$ parameter space to useful precision.

The $\chi^{2}$ statistic of equation (4) is well suited for determining the most likely values for the cosmological parameters $H_{0}, \Omega_{M}$, and $\Omega_{\Lambda}$ as well as the confidence intervals surrounding them. For constraining regions of parameter space not bounded by contours of uniform confidence (i.e., constant $\chi^{2}$ ), we need to define the probability density function (PDF) for the cosmological parameters. The PDF ( $p$ ) of these parameters given our distance moduli is derived from the PDF of the distance moduli given our data from Bayes's theorem,

$$
p\left(H_{0}, \Omega_{m}, \Omega_{\Lambda} \mid \mu_{0}\right)=\frac{p\left(\mu_{0} \mid H_{0}, \Omega_{m}, \Omega_{\Lambda}\right) p\left(H_{0}, \Omega_{m}, \Omega_{\Lambda}\right)}{p\left(\mu_{0}\right)},
$$

where $\mu_{0}$ is our set of distance moduli (Lupton 1993). Since we have no prior constraints on the cosmological parameters (besides the excluded regions) or on the data, we take $p\left(H_{0}, \Omega_{m}, \Omega_{\Lambda}\right)$ and $p\left(\mu_{0}\right)$ to be constants. Thus, we have for the allowed region of $\left(H_{0}, \Omega_{m}, \Omega_{\Lambda}\right)$

$$
p\left(H_{0}, \Omega_{m}, \Omega_{\Lambda} \mid \mu_{0}\right) \propto p\left(\mu_{0} \mid H_{0}, \Omega_{m}, \Omega_{\Lambda}\right) .
$$

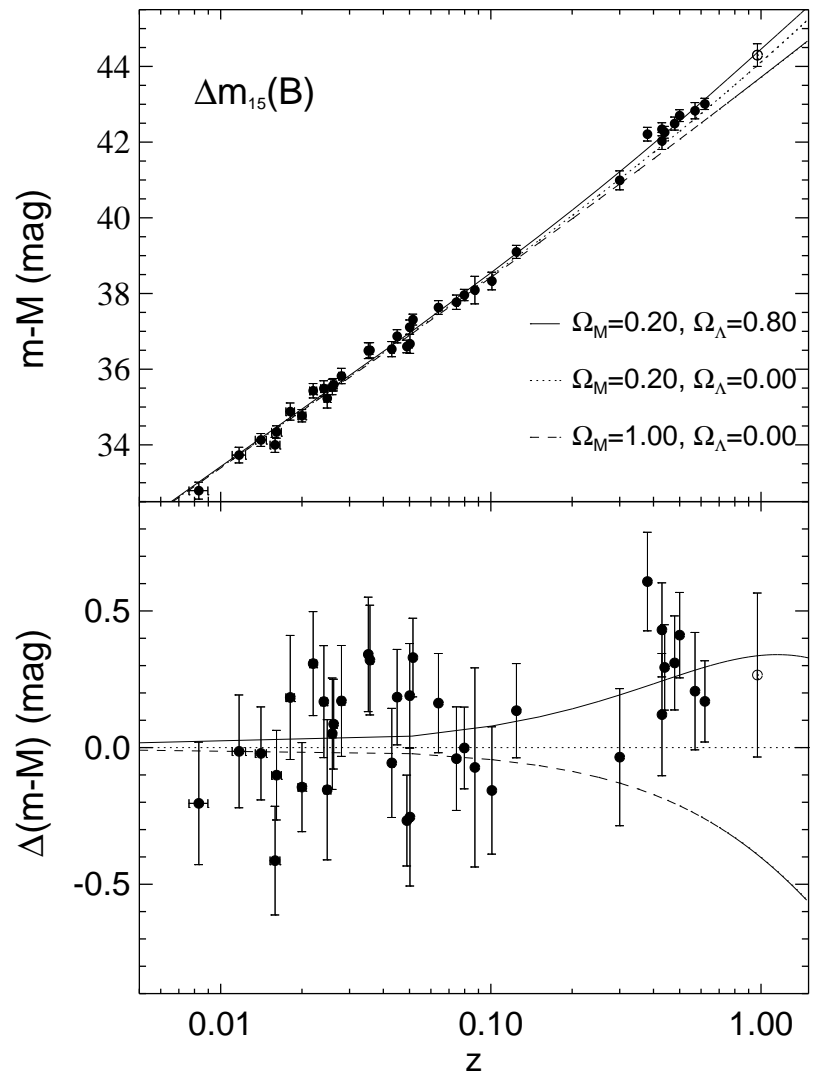

FIG. 5. $-\Delta m_{15}(B)$ SN Ia Hubble diagram. The upper panel shows the Hubble diagram for the low-redshift and high-redshift SNe Ia samples with distances measured from the template-fitting method parameterized by $\Delta m_{15}(B)$ (Hamuy et al. 1995, 1996d). Overplotted are three cosmologies: "low" and "high" $\Omega_{M}$ with $\Omega_{\Lambda}=0$ and the best fit for a flat cosmology, $\Omega_{M}=0.20, \Omega_{\Lambda}=0.80$. The bottom panel shows the difference between data and models from the $\Omega_{M}=0.20, \Omega_{\Lambda}=0$ prediction. The open symbol is SN 1997ck $(z=0.97)$, which lacks spectroscopic classification and a color measurement. The average difference between the data and the $\Omega_{M}=0.20, \Omega_{\Lambda}=0$ prediction is $0.28 \mathrm{mag}$.

We assume each distance modulus is independent (aside from systematic errors discussed in $\S 5$ ) and normally distributed, so the PDF for the set of distance moduli given the parameters is a product of Gaussians:

$$
\begin{aligned}
p\left(\mu_{0} \mid H_{0}, \Omega_{m}, \Omega_{\Lambda}\right) & =\prod_{i} \frac{1}{\sqrt{2 \pi\left(\sigma_{\mu_{0, i}}^{2}+\sigma_{v}^{2}\right)}} \\
& \times \exp \left\{-\frac{\left[\mu_{p, i}\left(z_{i} ; H_{0}, \Omega_{m}, \Omega_{\Lambda}\right)-\mu_{0, i}\right]^{2}}{2\left(\sigma_{\mu_{0, i}}^{2}+\sigma_{v}^{2}\right)}\right\} .
\end{aligned}
$$

Rewriting the product as a summation of the exponents and combining with equation (4), we have

$$
p\left(\mu_{0} \mid H_{0}, \Omega_{m}, \Omega_{\Lambda}\right)=\left[\prod_{i} \frac{1}{\sqrt{2 \pi\left(\sigma_{\mu_{0, i}}^{2}+\sigma_{v}^{2}\right)}}\right] \exp \left(-\frac{\chi^{2}}{2}\right) \text {. }
$$

The product in front is a constant, so combining with equation (6) the PDF for the cosmological parameters yields the standard expression (Lupton 1993)

$$
p\left(H_{0}, \Omega_{m}, \Omega_{\Lambda} \mid \mu_{0}\right) \propto \exp \left(-\frac{\chi^{2}}{2}\right) .
$$




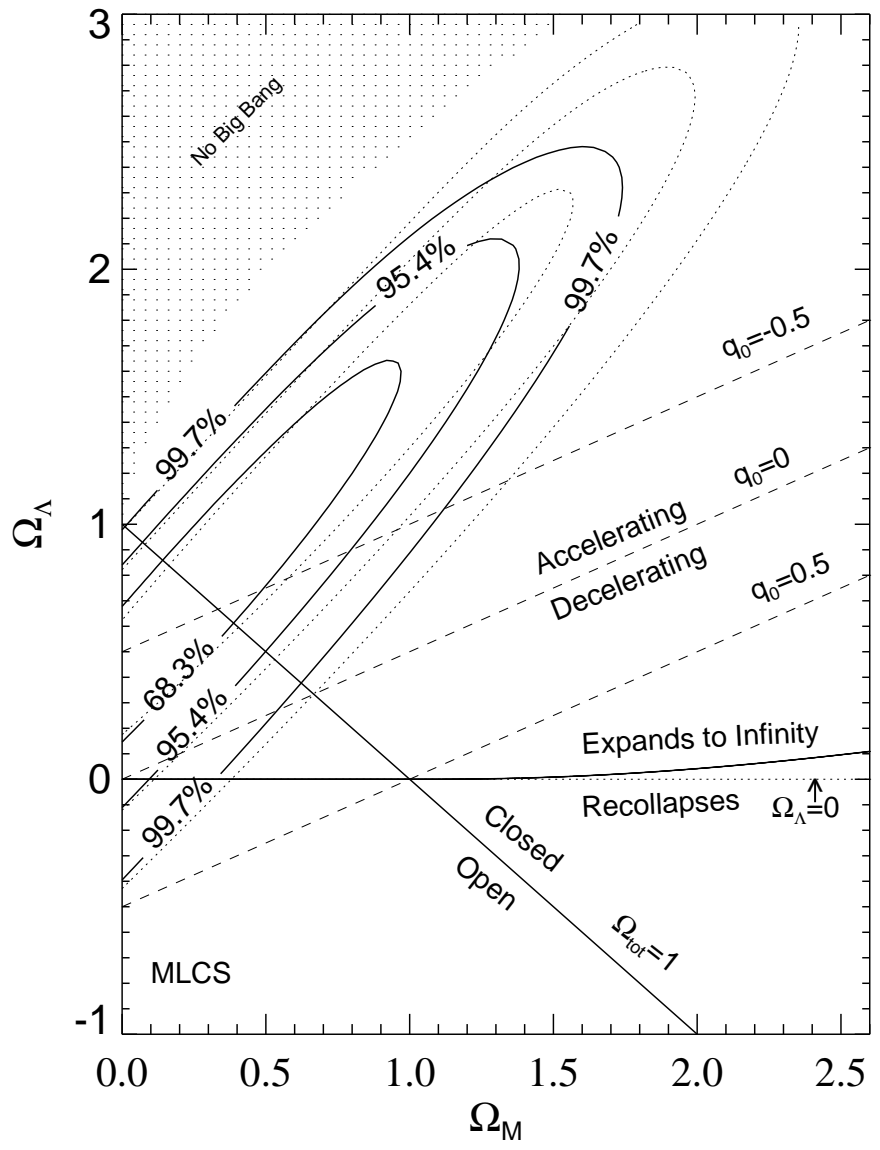

Fig. 6.-Joint confidence intervals for $\left(\Omega_{M}, \Omega_{\Lambda}\right)$ from SNe Ia. The solid contours are results from the MLCS method applied to well-observed SNe Ia light curves together with the snapshot method (Riess et al. 1998b) applied to incomplete SNe Ia light curves. The dotted contours are for the same objects excluding the unclassified SN 1997ck $(z=0.97)$. Regions representing specific cosmological scenarios are illustrated. Contours are closed by their intersection with the line $\Omega_{M}=0$.

The normalized PDF comes from dividing this relative PDF by its sum over all possible states,

$$
\begin{aligned}
p\left(H_{0}, \Omega_{m}, \Omega_{\Lambda} \mid \mu_{0}\right) & \\
& =\frac{\exp \left(-\chi^{2} / 2\right)}{\int_{-\infty}^{\infty} d H_{0} \int_{-\infty}^{\infty} d \Omega_{\Lambda} \int_{0}^{\infty} \exp \left(-\chi^{2} / 2\right) d \Omega_{M}},
\end{aligned}
$$

neglecting the unphysical regions. The most likely values for the cosmological parameters and preferred regions of parameter space are located where equation (4) is minimized or, alternately, equation (10) is maximized.

The Hubble constants as derived from the MLCS method, $65.2 \pm 1.3 \mathrm{~km} \mathrm{~s}^{-1} \mathrm{Mpc}^{-1}$, and from the templatefitting approach, $63.8 \pm 1.3 \mathrm{~km} \mathrm{~s}^{-1} \mathrm{Mpc}^{-1}$, are extremely robust and attest to the consistency of the methods. These determinations include only the statistical component of error resulting from the point-to-point variance of the measured Hubble flow and do not include any uncertainty in the absolute magnitude of SN Ia. From three photoelectrically observed SNe Ia, SN 1972E, SN 1981B, and SN 1990N (Saha et al. 1994, 1997), the SN Ia absolute magnitude was calibrated from observations of Cepheids in the host galaxies. The calibration of the SN Ia magnitude from only three objects adds an additional $5 \%$ uncertainty to the Hubble constant, independent of the uncertainty in the zero point of the distance scale. The uncertainty in the Cepheid distance scale adds an uncertainty of $\sim 10 \%$ to the derived Hubble constant (Feast \& Walker 1987; Kochanek 1997; Madore \& Freedman 1998). A realistic determination of the Hubble constant from SNe Ia would give $65 \pm 7 \mathrm{~km} \mathrm{~s}^{-1}$ $\mathrm{Mpc}^{-1}$, with the uncertainty dominated by the systematic uncertainties in the calibration of the SN Ia absolute magnitude. These determinations of the Hubble constant employ the Cepheid distance scale of Madore \& Freedman (1991), which uses a distance modulus to the Large Magellanic Cloud (LMC) of 18.50 mag. Parallax measurements by the Hipparcos satellite indicate that the LMC distance could be greater, and hence our inferred Hubble constant smaller, by $5 \%$ to $10 \%$ (Reid 1997), though not all agree with the interpretation of these parallaxes (Madore \& Freedman 1998). All subsequent indications in this paper for the cosmological parameters $\Omega_{M}$ and $\Omega_{\Lambda}$ are independent of the value for the Hubble constant or the calibration of the SN Ia absolute magnitude.

Indications for $\Omega_{M}$ and $\Omega_{\Lambda}$, independent from $H_{0}$, can be found by reducing our three-dimensional PDF to two dimensions. A joint confidence region for $\Omega_{M}$ and $\Omega_{\Lambda}$ is derived from our three-dimensional likelihood space

$$
p\left(\Omega_{M}, \Omega_{\Lambda} \mid \mu_{0}\right)=\int_{-\infty}^{\infty} p\left(\Omega_{M}, \Omega_{\Lambda}, H_{0} \mid \mu_{0}\right) d H_{0} .
$$

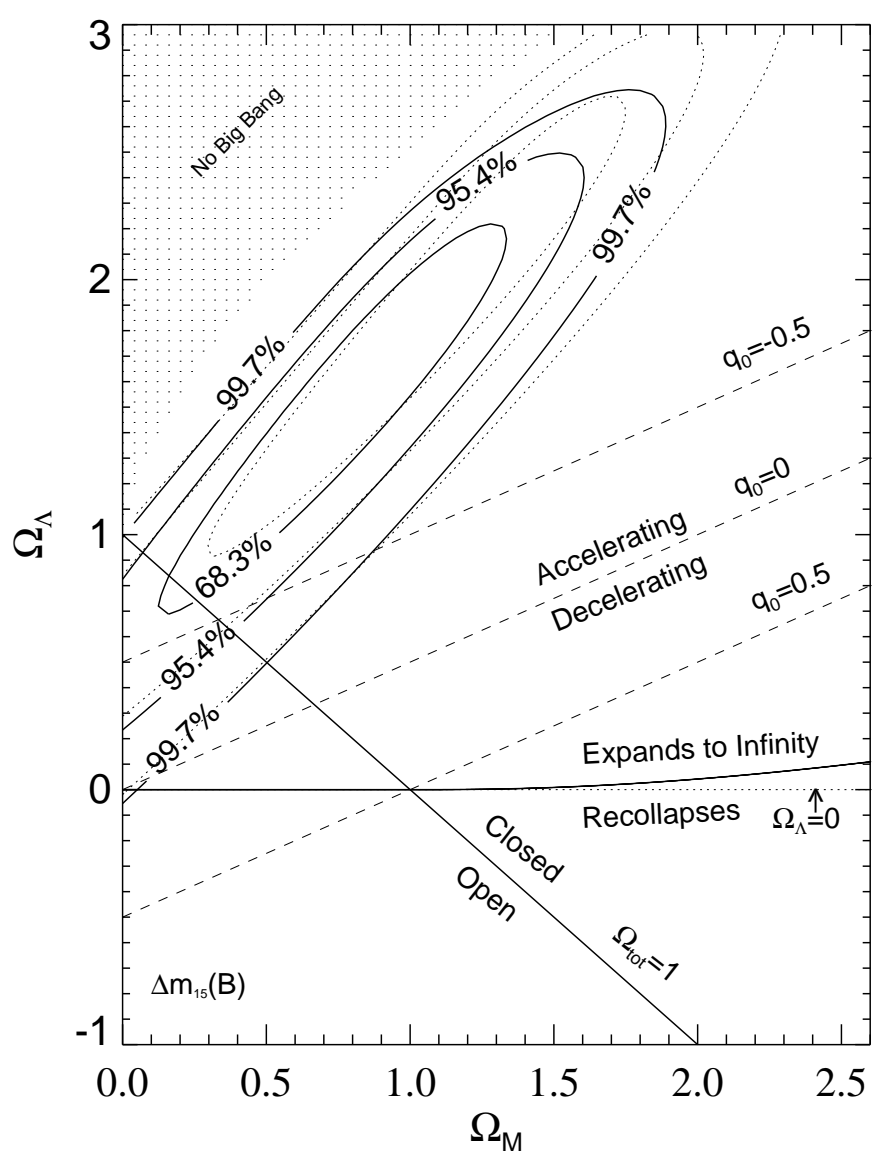

FIG. 7.- Joint confidence intervals for $\left(\Omega_{M}, \Omega_{\Lambda}\right)$ from SNe Ia. The solid contours are results from the template-fitting method applied to wellobserved SNe Ia light curves together with the snapshot method (Riess et al. 1998b) applied to incomplete SNe Ia light curves. The dotted contours are for the same objects excluding the unclassified SN 1997ck $(z=0.97)$. Regions representing specific cosmological scenarios are illustrated. Contours are closed by their intersection with the line $\Omega_{M}=0$. 
The likelihood that the cosmological constant is greater than zero is given by summing the likelihood for this region of parameter space:

$$
P\left(\Omega_{\Lambda}>0 \mid \mu_{0}\right)=\int_{0}^{\infty} d \Omega_{\Lambda} \int_{0}^{\infty} p\left(\Omega_{M}, \Omega_{\Lambda} \mid \mu_{0}\right) d \Omega_{M} .
$$

This integral was evaluated numerically over a wide and finely spaced grid of cosmological parameters for which equation (11) is nontrivial.

From the nine spectroscopic high-redshift $\mathrm{SNe}$ Ia with well-observed light and color curves, a non-negligible positive cosmological constant is strongly preferred at the $99.6 \%(2.9 \sigma)$ and greater than $99.9 \%(3.9 \sigma)$ confidence levels for the MLCS and template-fitting methods, respectively (see Table 8). This region of parameter space is nearly identical to the one that results in an eternally expanding universe. Boundless expansion occurs for a cosmological constant of

$$
\Omega_{\Lambda} \geq\left\{\begin{array}{l}
0, \\
4 \Omega_{M}\left\{\cos \left[\frac{1}{3} \cos ^{-1}\left(\frac{1-\Omega_{M}}{\Omega_{M}}\right)+\frac{4 \pi}{3}\right] \Omega_{M} \leq 1,\right.
\end{array}\right.
$$

(Carroll, Press, \& Turner 1992), and its likelihood is

$$
\begin{aligned}
\int_{0}^{1} d \Omega_{M} \int_{0}^{\infty} p\left(\Omega_{M}, \Omega_{\Lambda} \mid \mu_{0}\right) d \Omega_{\Lambda} \\
+\int_{1}^{\infty} d \Omega_{M} \int_{4 \Omega_{M}\left(\cos \left\{1 / 3 \cos ^{-1}\left[\left(1-\Omega_{M}\right) / \Omega_{M}\right]+(4 \pi / 3)\right\}\right\}^{3}}^{\infty} \\
p\left(\Omega_{M}, \Omega_{\Lambda} \mid \mu_{0}\right) d \Omega_{\Lambda} .
\end{aligned}
$$

The preference for eternal expansion is numerically equivalent to the confidence levels cited for a non-negligible, positive cosmological constant.

We can include external constraints on $\Omega_{M}, \Omega_{\Lambda}$, or their sum to further refine our determination of the cosmological parameters. For a spatially flat universe (i.e., $\Omega_{M}+\Omega_{\Lambda} \equiv$ $\left.\Omega_{\text {tot }} \equiv 1\right)$, we find $\Omega_{\Lambda}=0.68 \pm 0.10\left(\Omega_{M}=0.32 \pm 0.10\right)$ and $\Omega_{\Lambda}=0.84 \pm 0.09 \quad\left(\Omega_{M}=0.16 \pm 0.09\right)$ for MLCS and template fitting, respectively (see Table 8 ). The hypothesis that matter provides the closure density (i.e., $\Omega_{M}=1$ ) is ruled out at the $7 \sigma$ to $9 \sigma$ level by either method. Again, $\Omega_{\Lambda}>0$ and an eternally expanding universe are strongly preferred, at this same confidence level. We emphasize that these constraints reflect statistical errors only; systematic uncertainties are confronted in $\S 5$.

Other measurements based on the mass, light, X-ray emission, numbers, and motions of clusters of galaxies provide constraints on the mass density that have yielded typical values of $\Omega_{M} \approx 0.2-0.3$ (Carlberg et al. 1996; Bahcall, Fan, \& Cen 1997; Lin et al. 1996; Strauss \& Willick 1995). Using the constraint that $\Omega_{M} \equiv 0.2$ provides a significant indication for a cosmological constant: $\Omega_{\Lambda}=$ $0.65 \pm 0.22$ and $\Omega_{\Lambda}=0.88 \pm 0.19$ for the MLCS and template-fitting methods, respectively (see Table 8). For $\Omega_{M} \equiv 0.3$ we find $\Omega_{\Lambda}=0.80 \pm 0.22$ and $\Omega_{\Lambda}=0.96 \pm 0.20$ for the MLCS and template-fitting methods, respectively.

If we instead demand that $\Omega_{\Lambda} \equiv 0$, we are forced to relax the requirement that $\Omega_{M} \geq 0$ to locate a global minimum in our $\chi^{2}$ statistic. Doing so yields an unphysical value of
$\Omega_{M}=-0.38 \pm 0.22$ and $\Omega_{M}=-0.52 \pm 0.20$ for the MLCS and template-fitting approaches, respectively (see Table 8). This result emphasizes the need for a positive cosmological constant for a plausible fit.

For the four sparsely observed SNe Ia (SN 1996R, SN 1996T, SN 1995ao, and SN 1995ap), we employed the snapshot distance method (Riess et al. 1998b) to determine the luminosity distances. Unfortunately, the low priority given to these objects resulted in observations not only limited in frequency but in signal-to-noise ratio as well. Consequently, these four distances are individually uncertain at the $0.4-0.6$ mag level. We have compared these distances directly to a set of nine $\mathrm{SNe}$ Ia distances measured by the same snapshot method with $0.01 \leq z \leq 0.83$ from Riess et al. (1998b) and reprinted here in Tables 7 and 9. This approach avoids the requirement that distances calculated from light curves and the snapshot method be on the same distance scale, although this has been shown to be true (Riess et al. 1998b).

The complete but sparse set of 13 snapshot distances now including six SNe Ia with $z \geq 0.16$ yields conclusions that are less precise but fully consistent with the statistically independent results from the well-sampled SN Ia light curves (see Table 8).

Having derived the two PDFs, $p\left(\Omega_{M}, \Omega_{\Lambda}\right)$, for the $\sim 40$ $\mathrm{SNe}$ Ia light curves and the 13 incomplete ("snapshot") SNe Ia light curves independently, we can multiply the two PDFs to yield the PDF for all $\sim 50 \mathrm{SNe}$ Ia, which includes $15 \mathrm{SNe}$ with $0.16 \leq z \leq 0.62$. Contours of constant PDF from the MLCS method and the template-fitting method, each combined with the snapshot PDF, are shown in Figures 6 and 7. These contours are closed by their intersection with the line $\Omega_{M}=0$ and labeled by the total probability contained within.

Including the snapshot distances modestly strengthens all of the previous conclusions about the detection of a nonnegligible, positive cosmological constant (see Table 8). This set of 15 high-redshift SNe Ia favors $\Omega_{\Lambda} \geq 0$ and an eternally expanding universe at $99.7 \%(3.0 \sigma)$ and more than $99.9 \%(4.0 \sigma)$ confidence for the MLCS and template-fitting methods, respectively. This complete set of spectroscopic $\mathrm{SNe}$ Ia represents the full strength of the high-redshift sample and provides the most reliable results.

A remarkably high-redshift supernova $(z=0.97)$, SN $1997 \mathrm{ck}$, was excluded from all these analyses owing to its uncertain extinction and the absence of a spectroscopic identification. Nevertheless, if we assume a negligible extinction of $A_{B}=0.0 \pm 0.1$ for SN 1997ck as inferred for most of our high-redshift sample and further assume it is of Type Ia, as its well-observed $B$ rest-frame light curve suggests (see Fig. 3), we could include this object in our previous analysis (see Table 8). As seen in Figures 6 and 7, SN 1997ck constrains specific values of $\Omega_{M}$ and $\Omega_{\Lambda}$ by effectively closing our confidence contours because of the increased redshift range of this augmented sample. The values implied using SN 1997ck and the rest of the spectroscopic SNe Ia, under the previous assumptions, are $\Omega_{M}=0.24_{-0.24}^{+0.56}, \Omega_{\Lambda}=$ $0.72_{-0.48}^{+0.72}$ from the MLCS method and $\Omega_{M}=0.80_{-0.48}^{+0.40}$, $\Omega_{\Lambda}=1.56_{-0.70}^{+0.52}$ from the template-fitting method. The preference for a non-negligible, positive cosmological constant remains strong (see Table 8).

As seen in Table 8, the values of the $\chi_{v}^{2}$ for the cosmological fits are reassuringly close to unity. This statement is more meaningful for the MLCS distances, which are accompanied by statistically reliable estimates of the distance 


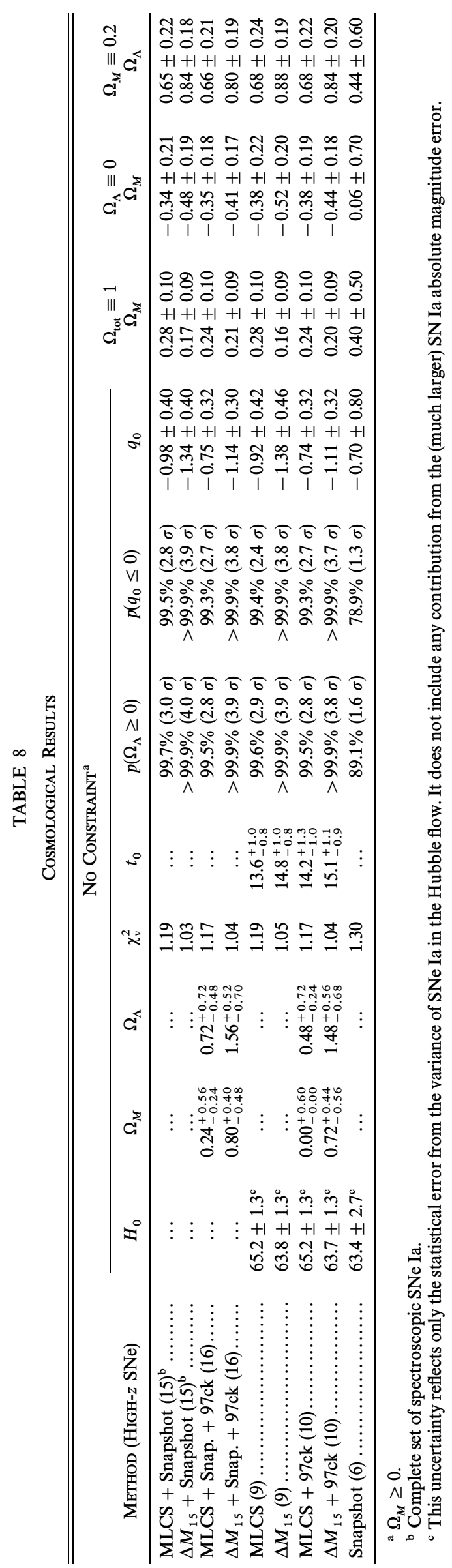


TABLE 9

NeArby SN Ia SNAPShot PARAMETERS ${ }^{\mathrm{a}}$

\begin{tabular}{ccrcc}
\hline \hline $\mathrm{SN}$ & $\log c z$ & \multicolumn{1}{c}{$\Delta$} & \multicolumn{1}{c}{$A_{V}$ Fit } & \multicolumn{1}{c}{$\mu_{0}\left(\sigma_{\mu_{0}}\right)$} \\
\hline $1994 \mathrm{U} \ldots \ldots$. & 3.111 & 0.03 & 0.70 & $31.72(0.10)$ \\
$1997 \mathrm{bp} \ldots \ldots$. & 3.363 & -0.26 & 0.62 & $32.81(0.10)$ \\
$1996 \mathrm{~V} \ldots \ldots$. & 3.870 & 0.26 & 0.00 & $35.35(0.17)$ \\
$1994 \mathrm{C} \ldots \ldots$. & 4.189 & 0.81 & 0.00 & $36.72(0.15)$ \\
$1995 \mathrm{M} \ldots \ldots$. & 4.202 & -0.15 & 0.46 & $37.12(0.15)$ \\
$1995 \mathrm{ae} \ldots \ldots$. & 4.308 & 0.38 & 0.00 & $37.58(0.21)$ \\
$1994 \mathrm{~B} \ldots \ldots$. & 4.431 & -0.02 & 0.38 & $38.51(0.10)$ \\
\hline
\end{tabular}

${ }^{\text {a }}$ As given in Riess et al. 1998b.

uncertainty (Riess et al. 1996a). The values for $\chi_{v}^{2}$ indicate a good agreement between the expected distance uncertainties and the observed distance dispersions around the best-fit model. They leave little room for sources of additional variance, as might be introduced by a significant difference between the properties of SNe Ia at high and low redshift.

\subsection{Deceleration Parameter}

An alternate approach to exploring the expansion history of the universe is to measure the current $(z=0)$ deceleration parameter, $q_{0} \equiv-\ddot{a}\left(t_{0}\right) a\left(t_{0}\right) / \dot{a}^{2}\left(t_{0}\right)$, where $a$ is the cosmic scale factor. Because the deceleration is defined at the current epoch and the supernovae in our sample cover a wide range in redshift, we can only determine the value of $q_{0}$ within the context of a model for its origin. Nevertheless, for moderate values of deceleration (or acceleration) the determination of $q_{0}$ from our $\mathrm{SNe}$, all of which are at $z<1$, provides a valuable description of the current deceleration parameter valid for most equations of state of the universe.

We have derived estimates of $q_{0}$ within a two-component model where $q_{0}=\left(\Omega_{M} / 2\right)-\Omega_{\Lambda}$. This definition assumes that the only sources of the current deceleration are mass density and the cosmological constant. A more complete definition for $q_{0}$ would include all possible forms of energy density (see Caldwell, Dave, \& Steinhardt 1998) but is beyond the scope of this paper. From our working definition of $q_{0}$, negative values for the current deceleration (i.e., accelerations) are generated only by a positive cosmological constant and not from unphysical, negative mass density.

Current acceleration of the expansion occurs for a cosmological constant of

$$
\Omega_{\Lambda} \geq \frac{\Omega_{M}}{2},
$$

and its likelihood is

$$
P\left(q_{0}<0 \mid \mu_{0}\right)=\int_{0}^{\infty} d \Omega_{M} \int_{\Omega_{M} / 2}^{\infty} p\left(\Omega_{M}, \Omega_{\Lambda} \mid \mu_{0}\right) d \Omega_{\Lambda}
$$

considering only $\Omega_{M} \geq 0$. Figures 6 and 7 show the boundary between current acceleration and deceleration as well as lines of constant $q_{0}$. For the complete set of supernova distances (excluding SN 1997ck), current acceleration is strongly preferred at the $99.5 \%(2.8 \sigma)$ confidence level for the MLCS method and a level of more than $99.9 \%(3.9 \sigma)$ for the template-fitting approach. The most likely value for $q_{0}$ is given by the peak of the distribution:

$$
p\left(q_{0} \mid \boldsymbol{\mu}_{0}\right)=\int_{0}^{\infty} d \Omega_{M} \int_{-\infty}^{\infty} p\left(\Omega_{M}, \Omega_{\Lambda} \mid q_{0}, \mu_{0}\right) d \Omega_{\Lambda} .
$$

This expression determines the likelihood of a given $q_{0}$ from the sum of the likelihoods of the combinations of $\Omega_{M}$ and $\Omega_{\Lambda}$ which produce that value of $q_{0}$. Values for $q_{0}$ and their uncertainties for the different methods and sample cuts are summarized in Table 8. With the current sample we find a robust indication for the sign of $q_{0}$ and a more uncertain estimate for its value, $q_{0}=-1.0 \pm 0.4$. Because lines of constant $q_{0}$ are skewed with respect to the major axis of our uncertainty contours, more SNe Ia at redshifts greater than $z=0.5$ will be needed to yield a more robust indication for the value of $q_{0}$.

\subsection{Dynamical Age of the Universe}

The dynamical age of the universe can be calculated from the cosmological parameters. In an empty universe with no cosmological constant, the dynamical age is simply the inverse of the Hubble constant; there is no deceleration. SNe Ia have been used to map the nearby Hubble flow, resulting in a precise determination of the Hubble constant (Hamuy et al. 1995, 1996c; Riess et al. 1995, 1996a). For a more complex cosmology, integrating the velocity of the expansion from the current epoch $(z=0)$ to the beginning $(z=\infty)$ yields an expression for the dynamical age

$$
\begin{array}{r}
t_{0}\left(H_{0}, \Omega_{M}, \Omega_{\Lambda}\right)=H_{0}^{-1} \int_{0}^{\infty}(1+z)^{-1}\left[(1+z)^{2}\left(1+\Omega_{M} z\right)\right. \\
\left.-z(2+z) \Omega_{\Lambda}\right]^{-1 / 2} d z
\end{array}
$$

(Carroll et al. 1992). Combining a PDF for the cosmological parameters, $p\left(H_{0}, \Omega_{M}, \Omega_{\Lambda}\right)$, with the above expression we can derive the PDF for the age of the universe:

$$
\begin{aligned}
p\left(t_{0} \mid \boldsymbol{\mu}_{0}\right)= & \int_{-\infty}^{\infty} d H_{0} \int_{0}^{\infty} d \Omega_{M} \\
& \times \int_{-\infty}^{\infty} p\left(H_{0}, \Omega_{M}, \Omega_{\Lambda} \mid t_{0}, \mu_{0}\right) d \Omega_{\Lambda} .
\end{aligned}
$$

Equation (19) expresses the likelihood for a given age, $t_{0}$, as the sum of the likelihoods of all combinations of $H_{0}, \Omega_{M}$, and $\Omega_{\Lambda}$ that result in the given age. The peak of this function provides our maximum likelihood estimate for the dynamical age, $t_{0}$. Without $S N 1997 \mathrm{ck}$, the peak is at 13.6 $6_{-0.8}^{+1.0}$ Gyr from the MLCS PDF. For the template-fitting approach the peak occurs at $14.8_{-0.8}^{+1.0}$ Gyr. A naive combination of the two distributions yields an estimate of $14.2_{-0.8}^{+1.0} \mathrm{Gyr}$ adopting either method's uncertainty (see Fig. 8). Again, these errors include only the statistical uncertainties of the measurement. Including the systematic uncertainty of the Cepheid distance scale, which may be as much as $10 \%$, a reasonable estimate of the dynamical age would be $14.2 \pm 1.7$ Gyr.

An illuminating way to characterize the dynamical age independent of the Hubble constant is to measure the product $H_{0} t_{0}$. For the MLCS method, the template-fitting method, and the combination of the two, we find $H_{0} t_{0}$ to be $0.90,0.96$, and 0.93 , respectively. These values imply a substantially older universe for a given value of $H_{0}$, in better accordance with globular cluster ages than the canonical value of $H_{0} t_{0}=\frac{2}{3}$ for $\Omega_{M}=1$ and $\Omega_{\Lambda}=0$. Our determination of the dynamical age of the universe is consistent with the rather wide range of values of the ages using stellar theory or radioactive dating. Oswalt et al. (1996) have shown that the Galactic disk has a lower age limit of 9.5 Gyr measured from the cooling sequence of the white dwarfs. The radioactive dating of stars via the thorium and europium abundances gives a value of $15.2 \pm 3.7 \mathrm{Gyr}$ 


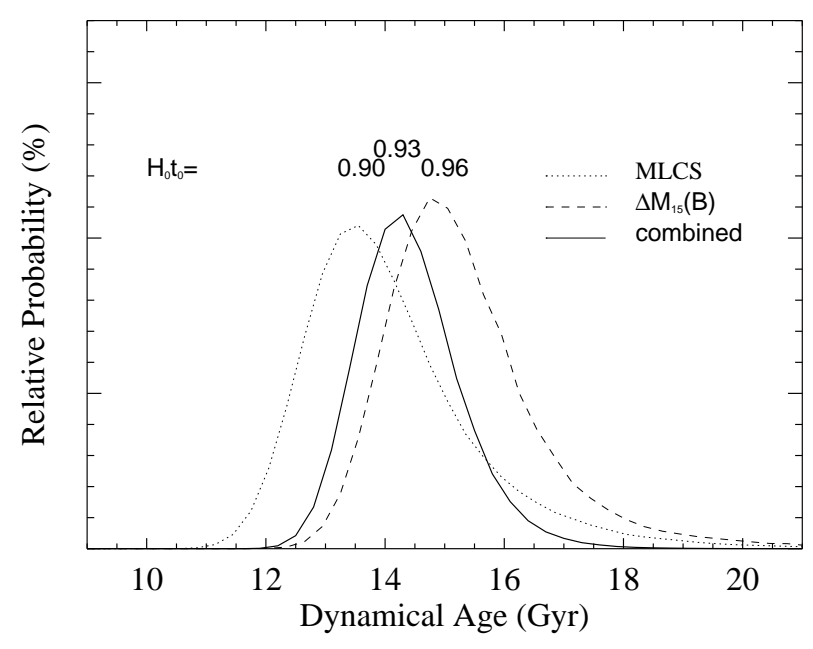

FIG. 8.-PDF for the dynamical age of the universe from $\mathrm{SNe}$ Ia (eq. [19]). The PDF for the dynamical age derived from the PDFs for $H_{0}, \Omega_{M}$, $\Omega_{\Lambda}$ is shown for the two different distance methods without the unclassified SN 1997ck. A naive average (see $\S 4.2$ ) yields an estimate of $14.2_{-0.8}^{+1.0} \mathrm{Gyr}$, not including the systematic uncertainties in the Cepheid distance scale.

(Cowan et al. 1997). We can expect these ages to become more precise as more objects are observed.

Perhaps the most widely quoted ages of the universe come from the age estimates of globular cluster stars. These are dependent on the distance scale used and the stellar

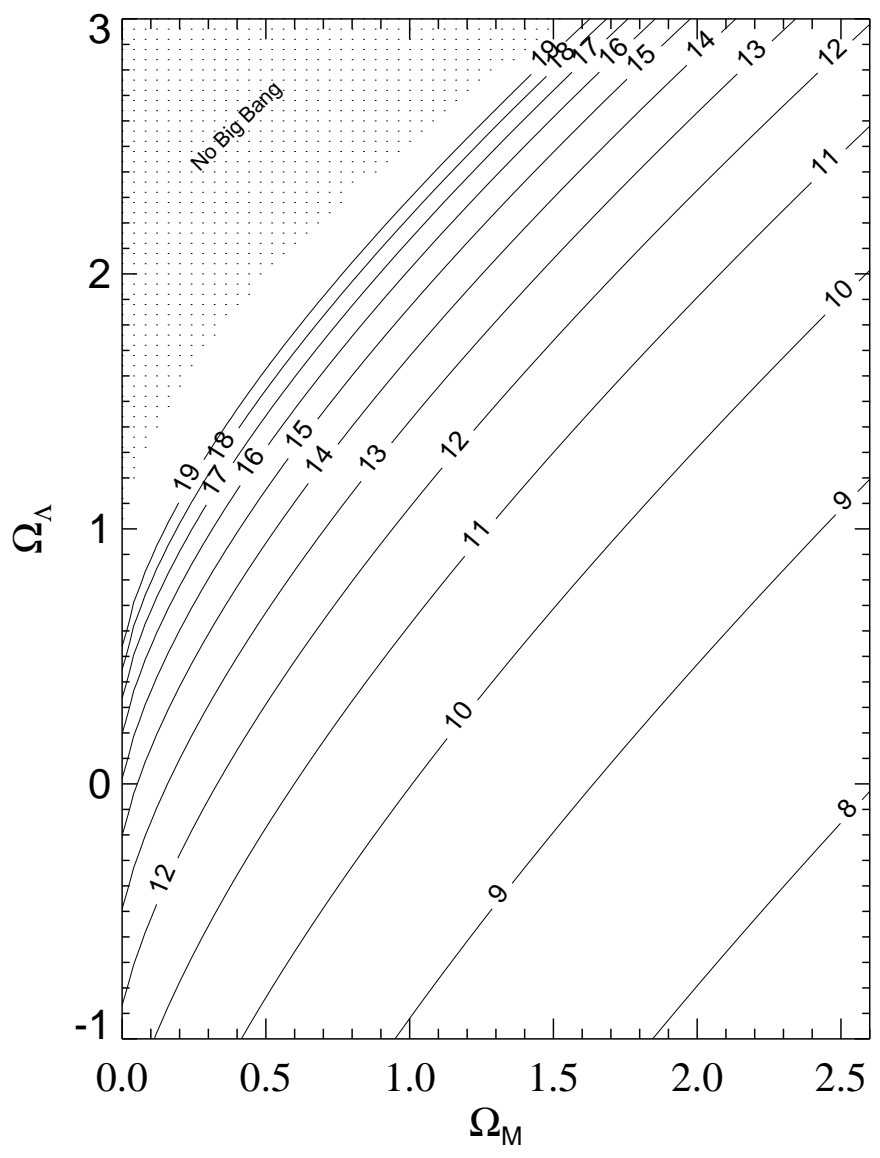

FIG. 9.-Lines of constant dynamical age in $\mathrm{Gyr}$ in the $\left(\Omega_{M}, \Omega_{\Lambda}\right)$-plane. Comparing these lines with the error ellipses in Figs. 5 and 7 reveals the leverage this experiment has on measuring the dynamical age. This plot assumes $H_{0}=65 \mathrm{~km} \mathrm{~s}^{-1} \mathrm{Mpc}^{-1}$ as determined from nearby SNe Ia and is subject to the zero point of the Cepheid distance scale. models employed. VandenBerg, Stetson, \& Bolte (1996) note that these two effects generally work in opposite directions: for instance, if one increases the distance to the LMC, the dynamical age of the universe increases, while the age based on the cluster ages decreases (the main-sequence turnoff is brighter, implying a younger population). This means that there is only a limited range in cosmological and stellar models that can bring the two ages into concordance.

Prior to Hipparcos, typical age estimates based on the subdwarf distance scale were greater than $15 \mathrm{Gyr}$ for cluster ages. Bolte \& Hogan (1995) find $15.8 \pm 2.1 \mathrm{Gyr}$ for the ages of the oldest clusters, while Chaboyer, Demarque, \& Sarajedini (1996) find a typical age of $18 \mathrm{Gyr}$ for the oldest clusters. Chaboyer (1995) also estimates the full range of viable ages to be 11-21 Gyr with the dominant error due to uncertainties in the theory of convection. An independent distance scale based on parallaxes of white dwarfs provides an age estimate for the globular cluster M4 of 14.5-15.5 Gyr (Renzini et al. 1996).

However, the Hipparcos parallaxes suggest an increased distance to the LMC and the globular clusters (Feast \& Catchpole 1997; Reid 1997; but see Madore \& Freedman 1998). With this new distance scale, the ages of the clusters have decreased to about $11.5 \mathrm{Gyr}$ with an uncertainty of 2 Gyr (Gratton et al. 1997; Chaboyer et al. 1998). Given the large range in ages from the theoretical models of cluster turnoffs and the inconsistency of the subdwarf and white dwarf distance scales applied to the ages of globular clusters, a robust estimate for the ages of the globular clusters remains elusive. Even with these uncertainties, the dynamical age of the universe derived here is consistent with the ages based on stellar theory or radioactive dating. Evidently, it is no longer a problem that the age of the oldest stars is greater than the dynamical age of the universe.

Despite our inability to place strong constraints on the values for $\Omega_{M}$ and $\Omega_{\Lambda}$ independently, our experiment is sensitive to the difference of these parameters. Because the dynamical age also varies approximately as the difference in $\Omega_{M}$ and $\Omega_{\Lambda}$, our leverage on the determination of the dynamical age is substantial. This point can be illustrated with a display of lines of constant dynamical age as a function of $\Omega_{M}$ and $\Omega_{\Lambda}$; comparing Figure 9 to Figures 6 and 7 , we see that the semimajor axes of our error ellipses are roughly parallel to the lines of constant dynamical age. Figure 9 also indicates why the most likely value for the dynamical age differs from the dynamical age derived for the most likely values of $H_{0}, \Omega_{M}$, and $\Omega_{\Lambda}$. For a fixed value of the Hubble constant, younger dynamical ages span a larger region of the $\left(\Omega_{M}, \Omega_{\Lambda}\right)$ parameter space than older ages. This shifts the most likely value for $t_{0}$ toward a younger age and results in a "tail" in the distribution, $p\left(t_{0} \mid \boldsymbol{\mu}_{0}\right)$, extending toward older ages.

\section{DISCUSSION}

The results of $\S 4$ suggest an eternally expanding universe that is accelerated by energy in the vacuum. Although these data do not provide independent constraints on $\Omega_{M}$ and $\Omega_{\Lambda}$ to high precision without ancillary assumptions or inclusion of a supernova with uncertain classification, specific cosmological scenarios can still be tested without these requirements.

High-redshift SNe Ia are observed to be dimmer than expected in an empty universe (i.e., $\Omega_{M}=0$ ) with no cosmological constant. A cosmological explanation for this obser- 
vation is that a positive vacuum energy density accelerates the expansion. Mass density in the universe exacerbates this problem, requiring even more vacuum energy. For a universe with $\Omega_{M}=0.2$, the MLCS and template-fitting distances to the well-observed $\mathrm{SNe}$ are 0.25 and $0.28 \mathrm{mag}$ farther on average than the prediction from $\Omega_{\Lambda}=0$. The average MLCS and template-fitting distances are still 0.18 and $0.23 \mathrm{mag}$ farther than required for a $68.3 \%(1 \sigma)$ consistency for a universe with $\Omega_{M}=0.2$ and without a cosmological constant.

Depending on the method used to measure all the spectroscopically confirmed SN Ia distances, we find $\Omega_{\Lambda}$ to be inconsistent with zero at confidence levels from $99.7 \%(3.0$ $\sigma$ ) to more than $99.9 \%(4.0 \sigma)$. Current acceleration of the expansion is preferred at the $99.5 \%(2.8 \sigma)$ to greater than $99.9 \%(3.9 \sigma)$ confidence level. The ultimate fate of the universe is sealed by a positive cosmological constant. Without a restoring force provided by a surprisingly large mass density (i.e., $\Omega_{M}>1$ ) the universe will continue to expand forever.

How reliable is this conclusion? Although the statistical inference is strong, here we explore systematic uncertainties in our results with special attention to those which can lead to overestimates of the $\mathrm{SNe}$ Ia distances.

\subsection{Evolution}

The local sample of $\mathrm{SNe}$ Ia displays a weak correlation between light curve shape (or luminosity) and host galaxy type. The sense of the correlation is that the most luminous SNe Ia with the broadest light curves only occur in latetype galaxies. Both early-type and late-type galaxies provide hosts for dimmer SNe Ia with narrower light curves (Hamuy et al. 1996b). The mean luminosity difference for $\mathrm{SNe} \mathrm{Ia}$ in late-type and early-type galaxies is $\sim 0.3 \mathrm{mag}$ (Hamuy et al. 1996b). In addition, the SN Ia rate per unit luminosity is almost twice as high in late-type galaxies as in early-type galaxies at the present epoch (Cappellaro et al. 1997). This suggests that a population of progenitors may exist in late-type galaxies that is younger and gives rise to brighter SNe Ia (with broader light curves) than those contained in early-type galaxies or within pockets of an older stellar population in the late-type galaxies. Such observations could indicate an evolution of $\mathrm{SNe}$ Ia with progenitor age.

Höflich, Wheeler, \& Thielemann (1998) calculate differences in the light curve shape, luminosity, and spectral characteristics of SNe Ia as a function of the initial composition and metallicity of the white dwarf progenitor. As we observe more distant samples, we expect the progenitors of SN Ia to come from a younger and more metal-poor population of stars. Höflich et al. (1998) have shown that a reduction in progenitor metallicity by a factor of 3 has little effect on the SN Ia bolometric luminosity at maximum. For their models, such a change in metallicity can alter the peak luminosity by small amounts $(\sim 0.05 \mathrm{mag})$ in rest-frame $B$ and $V$, accompanied by detectable spectral signatures. These spectral indicators of evolution are expected to be most discernible in the rest-frame $U$ passband, where line blanketing is prevalent. Future detailed spectral analyses at these short wavelengths might provide a constraint on a variation in progenitor metallicity.

The effect of a decrease in SN Ia progenitor age at high redshift is predicted to be more significant than metallicity (Höflich et al. 1998). Younger white dwarfs are expected to evolve from more massive stars with a lower ratio of $\mathrm{C} / \mathrm{O}$ in their cores. The lower $\mathrm{C} / \mathrm{O}$ ratio of the white dwarf reduces the amount of ${ }^{56} \mathrm{Ni}$ synthesized in the explosion, but an anticipated slower rise to maximum conserves more energy for an increased maximum brightness. By reducing the $\mathrm{C} / \mathrm{O}$ ratio from $1: 1$ to $2: 3$, the $B-V$ color at maximum is expected to become redder by 0.02 mag and the postmaximum decline would become steeper. This prediction of a brighter SN Ia exhibiting a faster postmaximum decline is opposite to what is seen in the nearby sample (Phillips 1993; Hamuy et al. 1995 1996a, 1996b, 1996c, 1996d; Riess et al. 1996a; Appendix) and will be readily testable for an enlarged high-redshift sample. Specifically, a larger sample of distant SNe Ia (currently being compiled) would allow us to determine the light curve shape relations at high redshift and test whether these evolve with look-back time. Presently, our sample is too small to make such a test meaningful.

We expect that the relation between light curve shape and luminosity that applies to the range of stellar populations and progenitor ages encountered in the late-type and early-type hosts in our nearby sample should also be applicable to the range we encounter in our distant sample. In fact, the range of age for SN Ia progenitors in the nearby sample is likely to be larger than the change in mean progenitor age over the 4-6 Gyr look-back time to the highredshift sample. Thus, to first order at least, our local sample should correct our distances for progenitor or age effects.

We can place empirical constraints on the effect that a change in the progenitor age would have on our SN Ia distances by comparing subsamples of low-redshift $\mathrm{SNe}$ Ia believed to arise from old and young progenitors. In the nearby sample, the mean difference between the distances for the early-type (eight SNe Ia) and late-type hosts (19 SNe Ia), at a given redshift, is $0.04 \pm 0.07 \mathrm{mag}$ from the MLCS method. This difference is consistent with zero. Even if the SN Ia progenitors evolved from one population at low redshift to the other at high redshift, we still would not explain the surplus in mean distance of 0.25 mag over the $\Omega_{\Lambda}=0$ prediction. For the template-fitting approach, the mean difference in distance for SNe Ia in early-type and late-type hosts is $0.05 \pm 0.07$ mag. Again, evolution provides an inadequate explanation for the $0.28 \mathrm{mag}$ difference in the template-fitting SNe Ia distances and the $\Omega_{\Lambda}=0$ prediction.

However, the low-redshift sample is dominated by latetype hosts, and these may contain a number of older progenitors. It is therefore difficult to assess the precise effect of a decrease in progenitor age at high redshift from the consistency of distances to early-type and late-type hosts (see Schmidt et al. 1998). If, however, we believed that young progenitors give rise to brighter $\mathrm{SNe}$ Ia with broader light curves (Hamuy et al. 1996b) as discussed above, we could more directly determine the effect on distance determinations of drawing our high-redshift sample from an increasingly youthful population of progenitors. The mean difference in the Hubble line defined by the full nearby sample and the subsample of SNe Ia with broader than typical light curves $(\Delta<0)$ is $0.02 \pm 0.07$ for the MLCS method. For the template-fitting method, the difference between the full sample and those with broader light curves $\left[\Delta m_{15}(B)<1.1\right]$ is $0.07 \pm 0.07$. Again, we find no indication of a systematic change in our distance estimates with a property that may correspond to a decrease in progenitor age. Another valuable test would be to compare low- 
redshift distances to starburst and irregular-type galaxies, which presumably are hosts to progenitors that are young and metal poor. Such a nearby sample may yield the closest approximation to the $\mathrm{SNe}$ Ia observed at high redshift. Future work will be needed to gather this informative sample, which would be composed of objects such as SN 1972E in NGC 5253 (which we does fit the luminosity light curve shape relations; Hamuy et al. 1996a).

Another check on evolutionary effects is to test whether the distribution of light curve decline rates is similar between the nearby sample of supernovae and the highredshift sample. Figure 10 shows the observed distribution of the MLCS light curve shape parameters, $\Delta$, and the template-fitting parameters, $\Delta m_{15}(B)$, with redshift. A Kolmogorov-Smirnov test shows no significant difference in the distributions of the low- and high-redshift samples, but the sample is too small to be statistically significant. The actual difference in mean luminosity between the lowredshift and high-redshift samples implied by the light curve shapes is 0.02 mag by either method. We conclude that there is no obvious difference between the shapes of SNe Ia light curves at $z \approx 0$ and at $z \approx 0.5$.

It is reassuring that initial comparisons of high-redshift SN Ia spectra appear remarkably similar to those observed at low-redshift. This can be seen in the high signal-to-noise
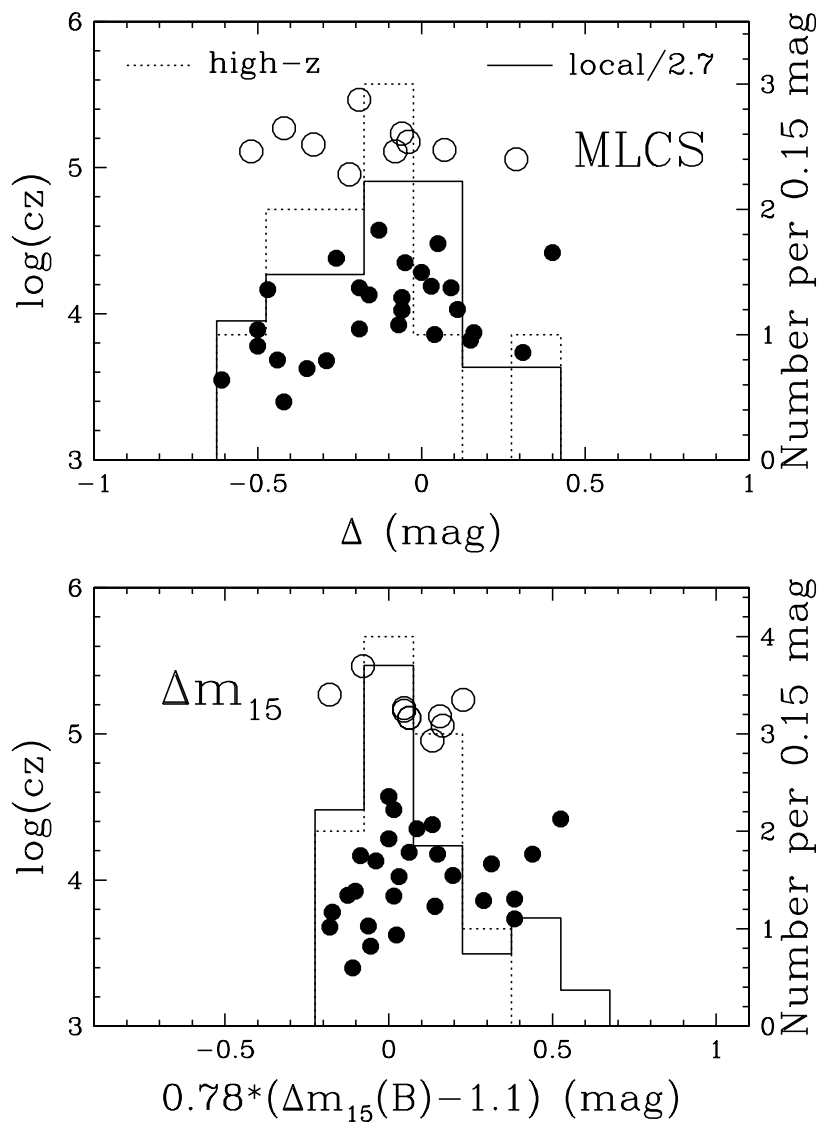

FIG. 10.-Distributions of MLCS light curve shape parameters, $\Delta$, and template-fitting parameters, $\Delta m_{15}(B)$, for the high- and low-redshift samples of SNe Ia. Positive values for $\Delta$ and $\Delta m_{15}(B)>1.1$ correspond to intrinsically dim SNe Ia, negative values for $\Delta$ and $\Delta m_{15}(B)<1.1$ correspond to luminous SNe Ia. Histograms of the low-redshift (solid line) and high-redshift (dotted line) light curve shape parameters are mutually consistent with no indication that these samples are drawn from different populations of SNe Ia. Filled and open circles show the distribution of $\log (c z)$ for the low- and high-redshift samples, respectively.

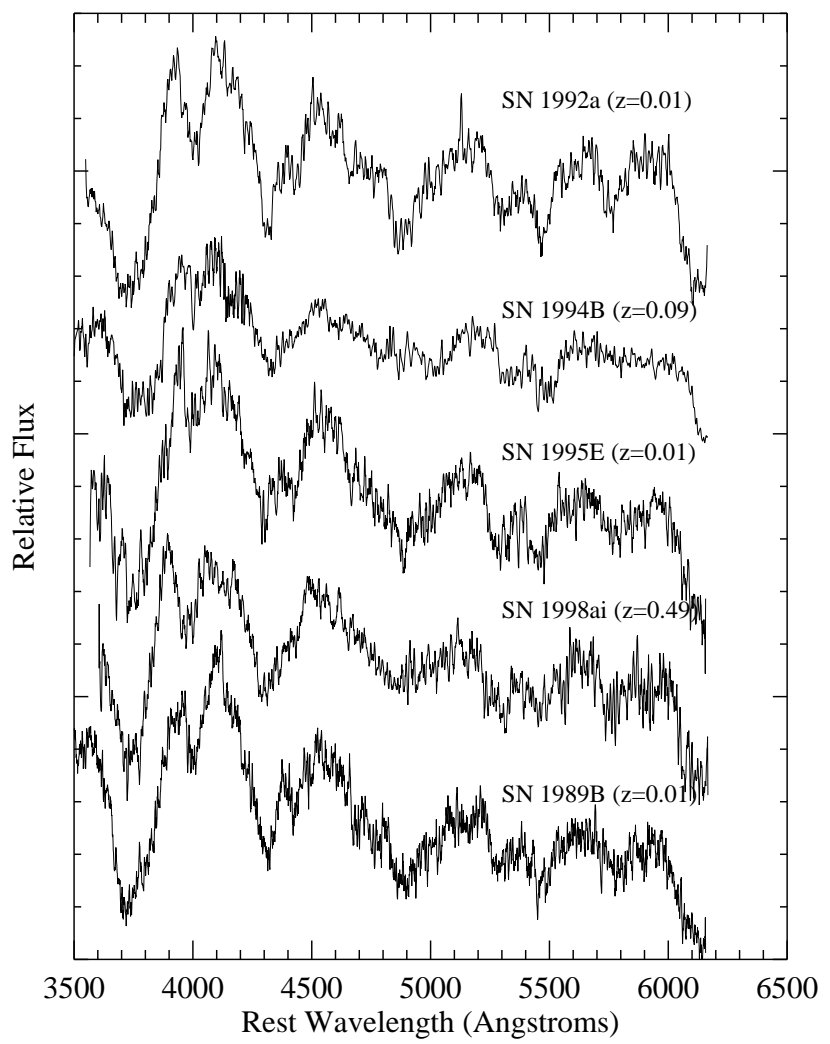

FIG. 11.-Spectral comparison (in $f_{\lambda}$ ) of SN 1998ai $(z=0.49)$ with lowredshift $(z<0.1) \mathrm{SNe}$ Ia at a similar age. Within the narrow range of SN Ia spectral features, SN 1998ai is indistinguishable from the low-redshift $\mathrm{SNe}$ Ia. The spectra from top to bottom are SN 1992A, SN 1994B, SN 1995E, SN 1998ai, and SN 1989B $\sim 5$ days before maximum light. The spectra of the low-redshift SNe Ia were resampled and convolved with Gaussian noise to match the quality of the spectrum of SN 1998ai.

ratio spectra of SN 1995ao $(z=0.30)$ and SN 1995ap $(z=0.23)$ in Figure 1. Another demonstration of this similarity at even higher redshift is shown in Figure 11 for SN 1998ai $(z=0.49$; Garnavich et al. 1996c), whose light curve was not used in this work. The spectrum of SN 1998ai was obtained at the Keck Telescope with a $5 \times 1800 \mathrm{~s}$ exposure using LRIS and was reduced as described in $\S 2.2$ (Filippenko et al. 1998). The spectral characteristics of this SN Ia appear to be indistinguishable from the range of characteristics at low redshift to good precision. In addition, a time sequence of spectra of SN Ia 1997ex $(z=0.36$; Nugent et al. 1998c) compared with those of local SNe Ia reveals no significant spectral differences (Filippenko et al. 1998).

We expect that our local calibration will work well at eliminating any pernicious drift in the supernova distances between the local and distant samples. Until we know more about the stellar ancestors of $\mathrm{SNe} \mathrm{Ia}$, we need to be vigilant for changes in the properties of the supernovae at significant look-back times. Our distance measurements could be particularly sensitive to changes in the colors of SNe Ia for a given light curve shape. Although our current observations reveal no indication of evolution of SNe Ia at $z \approx 0.5$, evolution remains a serious concern that can only be eased and perhaps understood by future studies.

\subsection{Extinction}

Our SNe Ia distances have the important advantage of including corrections for interstellar extinction occurring in 
the host galaxy and the Milky Way. The uncertainty in the extinctions is a significant component of error in our distance uncertainties. Extinction corrections based on the relation between $\mathrm{SN}$ Ia colors and luminosity improve distance precision for a sample of SNe Ia that includes objects with substantial extinction (Riess et al. 1996a). Yet, in practice, we have found negligible extinction to the high-redshift $\mathrm{SNe}$ Ia. The mean $B-V$ color at maximum is $-0.13 \pm 0.05$ from the MLCS method and $-0.07 \pm 0.05$ from the template-fitting approach, consistent with an unreddened $B-V$ color of -0.10 to -0.05 expected for slowly declining light curves as observed in the high-redshift sample (Riess et al. 1996a; Appendix).

Further, the consistency of the measured Hubble flow from SNe Ia with late-type and early-type hosts ( $§ 5.1)$ shows that the extinction corrections applied to dusty $\mathrm{SNe}$ Ia at low redshift do not alter the expansion rate from its value measured from $\mathrm{SNe}$ Ia in low dust environments. The conclusions reached in $\S 4$ would not alter if low- and highredshift $\mathrm{SNe}$ with significant extinction were discarded rather than included after a correction for extinction.

The results of $\S 4$ do not depend on the value of the ratios between color excess and selective absorption used to determine the extinctions of the high-redshift sample, because the mean observed reddening is negligible. Some modest departures from the Galactic reddening ratios have been observed in the Small and Large Magellanic Clouds, M31, and the Galaxy, and they have been linked to metallicity variations (Walterbos 1986; Hodge \& Kennicutt 1982; Bouchet et al. 1985; Savage \& Mathis 1979). Although our current understanding of the reddening ratios of interstellar dust at high redshift is limited, the lack of any significant color excess observed in the high-redshift sample indicates that the type of interstellar dust that reddens optical light is not obscuring our view of these objects.

Riess et al. (1996b) found indications that the Galactic ratios between selective absorption and color excess are similar for host galaxies in the nearby $(z \leq 0.1)$ Hubble flow. Yet, what if these ratios changed with look-back time? Could an evolution in dust grain size descending from ancestral interstellar "pebbles" at higher redshifts cause us to underestimate the extinction? Large dust grains would not imprint the reddening signature of typical interstellar extinction upon which our corrections rely. However, viewing our $\mathrm{SNe}$ through such gray interstellar grains would also induce a dispersion in the derived distances. To estimate the size of the dispersion, we assume that the gray extinction is distributed in galaxies in the same way as typical interstellar extinction.

Hatano et al. (1998) have calculated the expected distribution of SN Ia extinction along random lines of sight in the host galaxies. A gray extinction distribution similar to theirs could yield differing amounts of mean gray extinction depending on the likelihood assigned to observing an extinction of $A_{B}=0.0 \mathrm{mag}$. In the following calculations we vary only the likelihood of $A_{B}=0.0 \mathrm{mag}$ to derive new extinction distributions with varying means. These different distributions also have differing dispersions of extinction. A mean gray extinction of $0.25 \mathrm{mag}$ would be required to explain the measured MLCS distances without a cosmological constant. Yet the dispersion of individual extinctions for a distribution with a mean of $0.25 \mathrm{mag}$ would be $\sigma_{A_{B}}=$ 0.40 mag, significantly larger than the $0.21 \mathrm{mag}$ dispersion observed in the high-redshift MLCS distances. Gray extinc- tion is an even less likely culprit with the template-fitting approach; a distribution with a mean gray extinction of $0.28 \mathrm{mag}$, needed to replace a cosmological constant, would yield a dispersion of $0.42 \mathrm{mag}$, significantly higher than the distance dispersion of $0.17 \mathrm{mag}$ observed in the highredshift template-fitting distances.

Furthermore, most of the observed scatter is already consistent with the estimated statistical errors as evidenced by the $\chi_{v}^{2}$ (Table 8), leaving little to be caused by gray extinction. Nevertheless, if we assumed that all of the observed scatter were due to gray extinction, the mean shift in the $\mathrm{SNe}$ Ia distances would only be $0.05 \mathrm{mag}$. With the observations presented here, we cannot rule out this modest amount of gray interstellar extinction.

This argument applies not only to exotic gray extinction but to any interstellar extinction not accounted for which obscures SNe Ia. Any spotty interstellar extinction that varies with line of sight in a way similar to the Hatano et al. (1998) model of galaxies will add dispersion to the SN Ia distances. The low dispersion measured for the high-redshift sample places a strong limit on any mean spotty interstellar extinction.

Gray intergalactic extinction could dim the $\mathrm{SNe}$ without either telltale reddening or dispersion, if all lines of sight to a given redshift had a similar column density of absorbing material. The component of the intergalactic medium with such uniform coverage corresponds to the gas clouds producing $\operatorname{Ly} \alpha$ forest absorption at low redshifts. These clouds have individual $\mathrm{H}$ I column densities less than about $10^{15} \mathrm{~cm}^{-2}$ (Bahcall et al. 1996). However, these clouds display low metallicities, typically less than $10 \%$ of solar. Gray extinction would require larger dust grains, which would need a larger mass in heavy elements than typical interstellar grain size distributions to achieve a given extinction. Furthermore, these clouds reside in hard radiation environments hostile to the survival of dust grains. Finally, the existence of gray intergalactic extinction would only augment the already surprising excess of galaxies in highredshift galaxy surveys (Huang et al. 1997).

We conclude that gray extinction does not seem to provide an observationally or physically plausible explanation for the observed faintness of high-redshift SNe Ia.

\subsection{Selection Bias}

Sample selection has the potential to distort the comparison of nearby and distant supernovae. Most of our nearby $(z<0.1)$ sample of $\mathrm{SNe}$ Ia was gathered from the Calán/ Tololo survey (Hamuy et al. 1993a), which employed the blinking of photographic plates obtained at different epochs with Schmidt telescopes and from less well defined searches (Riess et al. 1998c). Our distant $(z>0.16)$ sample was obtained by subtracting digital CCD images at different epochs with the same instrument setup.

If they were limited by the flux of the detected events, both nearby and distant SN Ia searches would preferentially select intrinsically luminous objects because of the larger volume of space in which these objects can be detected. This well-understood selection effect could be further complicated by the properties of SNe Ia; more luminous supernovae have broader light curves (Phillips 1993; Hamuy et al. 1995, 1996b; Riess et al. 1995, 1996a). The brighter supernovae remain above a detection limit longer than their fainter siblings, but also can fail to rise above the detection limit in the time interval between successive 
search epochs. The complex process by which $\mathrm{SNe}$ Ia are selected in low- and high-redshift searches can be best understood with simulations (Hamuy \& Pinto 1998). Although selection effects could alter the ratio of intrinsically dim to bright $\mathrm{SNe}$ Ia in our samples relative to the true population, our use of the light curve shape to determine the supernova's luminosity should correct most of this selection bias on our distance estimates. However, even after our light curve shape correction, SNe Ia still have a small dispersion as distance indicators $(\sigma \approx 0.15 \mathrm{mag})$, and any search program would still preferentially select objects that are brighter than average for a particular light curve shape and possibly select objects whose light curve shapes aid detection.

To investigate the consequence of sample selection effects, we used a Monte Carlo simulation to understand how SNe Ia in our nearby and distant samples were chosen. For the purpose of this simulation, we first assumed that the SN Ia rate is constant with look-back time. We assembled a population of SNe Ia with luminosities described by a Gaussian random variable $\sigma_{M_{B}}=0.4 \mathrm{mag}$ and light curve shapes, which correspond to these luminosities as described by the MLCS vectors (see the Appendix). A Gaussian random uncertainty of $\sigma=0.15 \mathrm{mag}$ is assumed in the determination of absolute magnitude from the shape of a supernova's light curve. The time interval between successive search epochs, the search epoch's limiting magnitudes, and the apparent light curve shapes were used to determine which SNe Ia were "discovered" and included in the simulation sample. A separate simulation was used to select nearby objects, with the appropriate time interval between epochs and estimates of limiting magnitudes. The results are extremely encouraging, with recovered values exceeding the simulated value of $\Omega_{M}$ or $\Omega_{\Lambda}$ by only 0.02 for these two parameters considered separately. Smoothly increasing the SN Ia rate by a factor of 10 by $z=1$ doubles this bias to 0.04 for either parameter. There are two reasons we find such a small selection bias in the recovered cosmological parameters. First, the small dispersion of our distance indicator results in only a modest selection bias. Second, both nearby and distant samples include an excess of brighter than average $\mathrm{SNe}$, so the difference in their individual selection biases remains small.

As discussed by Schmidt et al. (1998), obtaining accurate limiting magnitudes is complex for the CCD-based searches, and essentially impossible for the photographic searches. Limiting magnitudes vary from frame to frame, night to night, and film to film, so it is difficult to use the actual detection limits in our simulation. Nevertheless, we have run simulations varying the limiting magnitude, and this does not change the results significantly. We have also tried increasing the dispersion in the SN Ia light curve shape versus absolute magnitude correlation at wavelengths shorter than $5000 \AA$. Even doubling the distance dispersion of $\mathrm{SNe}$ Ia (as may be the case for rest-frame $U$ ) does not significantly change the simulation results.

Although these simulations bode well for using SNe Ia to measure cosmological parameters, there are other differences between the way nearby and distant supernova samples are selected that are more difficult to model and are not included in our present simulations. Von Hippel, Bothun, \& Schommer (1997) have shown that the selection function of the nearby searches is not consistent with that of a strict magnitude-limited search. It is unclear whether a photographic search selects SNe Ia with different parameters or environments than a CCD search or how this could affect a comparison of samples. Future work on quantifying the selection criteria of the samples is needed. A CCD search for SNe Ia in Abell clusters by Reiss et al. (1998a) will soon provide a nearby SN Ia sample with better understood selection criteria. Although indications from the distributions of SN Ia parameters suggest that both our searches have sampled the same underlying population (see Fig. 10), we must continue to be wary of subtle selection effects that might bias the comparison of SNe Ia near and far.

\subsection{Effect of a Local Void}

It has been noted by Zehavi et al. (1998) that the SNe Ia out to $7000 \mathrm{~km} \mathrm{~s}^{-1}$ exhibit an expansion rate that is $6 \%$ greater than that measured for the more distant objects. The significance of this peculiar monopole is at the $2 \sigma$ to 3 $\sigma$ confidence level; it is not inconsistent with the upper limit of $\sim 10 \%$ for the difference between the local and global values of $H_{0}$ found by Kim et al. (1997). The implication is that the volume out to this distance is underdense relative to the global mean density. This effect appears as an excess redshift for a given distance modulus (within $7000 \mathrm{~km} \mathrm{~s}^{-1}$ ) and can be seen with both the MLCS method and the template-fitting method in Figures 4 and 5.

If true, what effect would this result have on our conclusions? In principle, a local void would increase the expansion rate measured for our low-redshift sample relative to the true, global expansion rate. Mistaking this inflated rate for the global value would give the false impression of an increase in the low-redshift expansion rate relative to the high-redshift expansion rate. This outcome could be incorrectly attributed to the influence of a positive cosmological constant. In practice, only a small fraction of our nearby sample is within this local void, reducing its effect on the determination of the low-redshift expansion rate.

As a test of the effect of a local void on our constraints for the cosmological parameters, we reanalyzed the data discarding the seven SNe Ia within $7000 \mathrm{~km} \mathrm{~s}^{-1}(108 \mathrm{Mpc}$ for $\left.H_{0}=65\right)$. The result was a reduction in the confidence that $\Omega_{\Lambda}>0$ from $99.7 \%(3.0 \sigma)$ to $98.3 \%(2.4 \sigma)$ for the MLCS method and from more than $99.9 \%(4.0 \sigma)$ to $99.8 \%(3.1 \sigma)$ for the template-fitting approach. The tests for both methods excluded the unclassified SN 1997ck and included the snapshot sample, the latter without two $\mathrm{SNe}$ Ia within $7000 \mathrm{~km} \mathrm{~s}^{-1}$. As expected, the influence of a possible local void on our cosmological conclusions is relatively small.

\subsection{Weak Gravitational Lensing}

The magnification and demagnification of light by largescale structure can alter the observed magnitudes of highredshift supernovae (Kantowski, Vaughan, \& Branch 1995). The effect of weak gravitational lensing on our analysis has been quantified by Wambsganss et al. (1997) and summarized by Schmidt et al. (1998). SN Ia light will, on average, be demagnified by $0.5 \%$ at $z=0.5$ and $1 \%$ at $z=1$ in a universe with a non-negligible cosmological constant. Although the sign of the effect is the same as the influence of a cosmological constant, the size of the effect is negligible.

Holz \& Wald (1998) have calculated the weak lensing effects on supernova light from ordinary matter, which is not smoothly distributed in galaxies but rather clumped into stars (i.e., dark matter contained in MACHOs). With this scenario, microlensing by compact masses becomes a 
more important effect, further decreasing the observed supernova brightnesses at $z=0.5$ by $0.02 \mathrm{mag}$ for $\Omega_{M}=0.2$ (D. E. Holz 1998, private communication). Even if most ordinary matter were contained in compact objects, this effect would not be large enough to reconcile the SNe Ia distances with the influence of ordinary matter alone.

\subsection{Light Curve Fitting Method}

As described in $\S 3.2$, two different light curve fitting methods, MLCS (Riess et al. 1996a; Appendix) and a template-fitting approach (Hamuy et al. 1995, 1996d), were employed to determine the distances to the nearby and high-redshift samples. Both methods use relations between light curve shape and luminosity as determined from $\mathrm{SNe} \mathrm{Ia}$ in the nearby Hubble flow. Both methods employ an extinction correction from the measured color excess using relations between intrinsic color and light curve shape. In addition, both the MLCS and template-fitting methods yield highly consistent measurements for the Hubble constant of $H_{0}=65.2 \pm 1.3$ and $H_{0}=63.8 \pm 1.3$, respectively not including any uncertainty in the determination of the SN Ia absolute magnitude, which is the dominant uncertainty. It is also worth noting that both methods yield SN Ia distance dispersions of $\sim 0.15 \mathrm{mag}$ when complete light curves in $B, V, R$, and $I$ are employed. For the purpose of comparing the same data at high and low redshifts, the use of SN Ia observations at low redshift were restricted to only $B$ and $V$ within 40 days of maximum light.

Although the conclusions reached by the two methods when applied to the high-redshift $\mathrm{SNe}$ are highly consistent, some differences are worth noting. There are small differences in the distance predictions at high redshift. For the distant sample, the template-fitting distances exhibit a scatter of $0.17 \mathrm{mag}$ around the best-fit model as compared with $0.21 \mathrm{mag}$ for the MLCS method. In addition, the template-fitting distances to the high-redshift SNe Ia are on (weighted) average 0.03 mag farther than the MLCS distances relative to the low-redshift sample. These differences together result in slightly different confidence intervals for the two methods (see Figs. 6, 7, and 8 and Table 8). For the set of 10 well-observed SNe Ia, a sample with scatter 0.17 mag or less is drawn from a population of scatter $0.21 \mathrm{mag}$ $25 \%$ of the time. The chance that 10 objects could be drawn from this same population with a mean difference of 0.03 mag is $66 \%$. Future samples of SNe Ia will reveal if the observed differences are explained by chance. Until then, we must consider the difference between the cosmological constraints reached from the two fitting methods to be a systematic uncertainty. Yet, for the data considered here, both distance fitting methods unanimously favor the existence of a non-negligible, positive cosmological constant and an accelerating universe.

\subsection{Sample Contamination}

The mean brightness of SNe Ia is typically 4-40 times greater than that of any other type of supernova, favoring their detection in the volume of space searched at high redshift. Yet in the course of our high-redshift supernova search (and that of the Supernova Cosmology Project; Perlmutter et al. 1995) a small minority of other supernova types have been found, and we must be careful not to include such objects in our SN Ia sample. The classification of a supernova is determined from the presence or absence of specific features in the spectrum (Wheeler \& Harkness 1990;
Branch, Fisher, \& Nugent 1993; Filippenko 1997). The spectra of Type Ia supernovae show broad Si II absorption near $6150 \AA$, Ca II (H and K) absorption near $3800 \AA$, an S II absorption doublet near 5300 and $5500 \AA$, and numerous other absorption features, with ionized $\mathrm{Fe}$ a major contributor (Filippenko 1997). For supernovae at high redshift, some of these characteristic features shift out of the observer's frequency range as other, shorter wavelength features become visible. Classification is further complicated by low signal-to-noise ratio in the spectra of distant objects. The spectra of SNe Ia evolve with time along a remarkably reliable sequence (Riess et al. 1997). Final spectral classification is optimized by comparing the observed spectrum to well-observed spectra of SNe Ia at the same age as determined from the light curves.

For most of the spectra in Figure 1, the identification as a $\mathrm{SN}$ Ia is unambiguous. However, in three of the lowest signal-to-noise ratio cases-1996E, $1996 \mathrm{H}$, and $1996 \mathrm{I}$ - the wavelengths near Si II absorption (rest frame $6150 \AA$ ) were poorly observed, and their classification warrants closer scrutiny. These spectra are inconsistent with Type II spectra, which show $\mathrm{H} \beta$ (4861 $\AA$ ) in emission and absorption and lack Fe II features shortly after maximum. These spectra are also inconsistent with Type Ib spectra, which would display He I $\lambda 5876$ absorption at a rest wavelength of $\sim 5700 \AA$.

The most likely supernova type to be misconstrued as a Type Ia is a Type Ic, as this type comes closest to matching the SN Ia spectral characteristics. Although SN Ic spectra lack Si II and S II absorption, the maximum-light spectra at blue wavelengths can resemble those of SNe Ia $\sim 2$ weeks past maximum, when both are dominated by absorption lines of Fe II with $\mathbf{P}$ Cygni profiles. Type Ic events are rare and one luminous enough to be found in our search would be rare indeed, but not without precedent. An example of such an object is SN 1992ar (Clocchiatti et al. 1998), which was discovered in the course of the Calán/Tololo SN survey and which reached an absolute magnitude, uncorrected for host galaxy dust extinction, of $M_{V}=-19.3\left(H_{0}=65 \mathrm{~km}\right.$ $\left.\mathrm{s}^{-1} \mathrm{Mpc}^{-1}\right)$. For both SN $1996 \mathrm{H}$ and SN 1996I, the spectral match with a Type Ia at rest wavelengths less than 4500 $\AA$ is superior to the fit to a Type Ic spectrum (see Fig. 1). In both cases the spectra rise from deep troughs at the $3800 \AA$ Ca II break (rest frame) to strong peaks at 3900-4100 $\AA$ (rest frame) as observed in SNe Ia. Type Ic spectra, by comparison, tend to exhibit a much weaker transition from trough to peak redward of the Ca II break (see Fig. 12).

For SN 1996E, the spectral coverage does not extend blueward of a rest wavelength of $4225 \AA$, rendering this diagnostic unusable. The absence of premaximum observations of SN 1996E makes it difficult to determine the age of the spectrum and that of the appropriate comparison spectra. As shown in Figure 12, the spectroscopic and photometric data for SN 1996E are consistent with a SN Ia caught $\sim 1$ week after maximum light, or a luminous SN Ic discovered at maximum. There is a weak indication of S II absorption at $\sim 5375 \AA$, which favors classification as a Type Ia (see Figs. 1 and 12), but this alone does not provide a secure classification. Note that the $K$-corrections for a SN Ia or SN Ic at this redshift $(z=0.43)$ would be nearly identical due to the excellent match of the observed filters (B45 and V45) to the rest-frame ( $B$ and $V$ ) filters.

We have reanalyzed the cosmological parameters discarding SN 1996E as a safeguard against the possible con- 

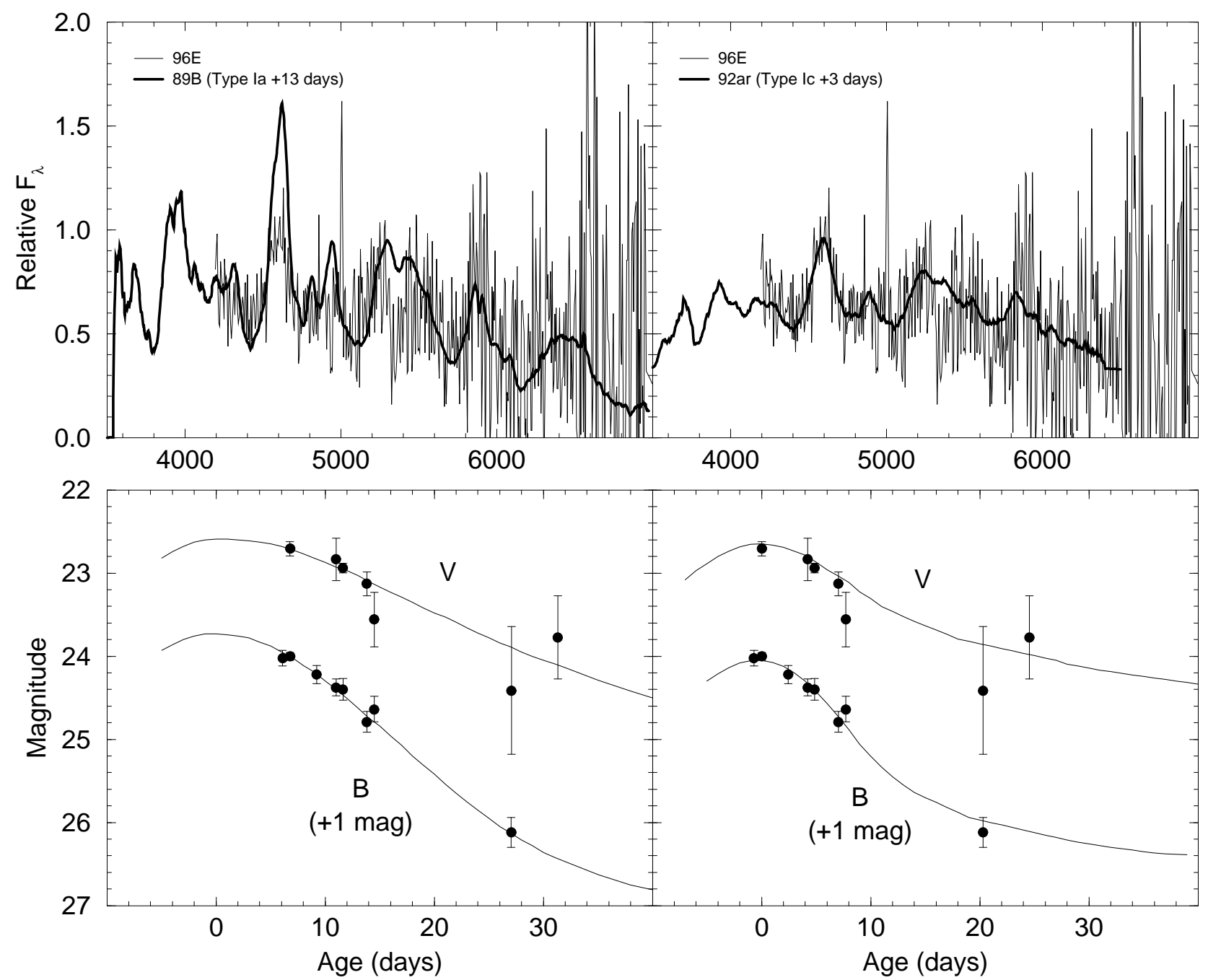

FIG. 12.-Comparison of the spectral and photometric observations of SN 1996E to those of Type Ia and Type Ic supernovae. The low signal-to-noise ratio of the spectrum of SN 1996E and the absence of data blueward of 4500 Å makes it difficult to distinguish between a Type Ia and Ic classification. The light and color curves of SN 1996E are also consistent with either supernova type. The spectrum was taken 6 days (rest frame) after the first photometric observation.

tamination of our high-redshift sample. We also excluded SN 1997ck, which, for lack of a definitive spectral classification, is an additional threat to contamination of our sample. With the remaining "high-confidence" sample of $14 \mathrm{SNe}$ Ia we find the statistical likelihood of a positive cosmological constant to be $99.8 \%(3.1 \sigma)$ from the MLCS method, a modest increase from $99.7 \%(3.0 \sigma)$ confidence when SN 1996E is included. For the template-fitting approach, the statistical confidence in a positive cosmological constant remains high at more than $99.9 \%(4.0 \sigma)$, the same result as with SN 1996E. We conclude that for this sample our results are robust against sample contamination, but the possible contamination of future samples remains a concern. Even given existing detector technology, more secure supernova classifications can be achieved with greater signal-to-noise ratios for observed spectra, with optimally timed search epochs that increase the likelihood of premaximum discovery, and with an improved empirical understanding of the differences among the spectra of supernova types.

\subsection{Comparisons}

The results reported here are consistent with other reported observations of high-redshift $\mathrm{SNe}$ Ia from the
High- $z$ Supernova Search Team (Garnavich et al. 1998a; Schmidt et al. 1998), and the improved statistics of this larger sample reveal the potential influence of a positive cosmological constant.

These results are inconsistent at the $\sim 2 \sigma$ confidence level with those of Perlmutter et al. (1997), who found $\Omega_{M}=0.94 \pm 0.3\left(\Omega_{\Lambda}=0.06\right)$ for a flat universe and $\Omega_{M}=$ $0.88 \pm 0.64$ for $\Omega_{\Lambda} \equiv 0$. They are marginally consistent with those of Perlmutter et al. (1998), who, with the addition of one very high redshift SN Ia $(z=0.83)$, found $\Omega_{M}=0.6$ $\pm 0.2\left(\Omega_{\Lambda}=0.4\right)$ for a flat universe and $\Omega_{M}=0.2 \pm 0.4$ for $\Omega_{\Lambda} \equiv 0$.

Although the experiment reported here is very similar to that performed by Perlmutter et al. $(1997,1998)$, there are some differences worth noting. Schmidt et al. (1998), Garnavich et al. (1998a), and this paper explicitly correct for the effects of extinction evidenced by reddening of the $\mathrm{SNe}$ Ia colors. Not correcting for extinction in the nearby and distant sample could affect the cosmological results in either direction since we do not know the sign of the difference of the mean extinction. In practice we have found few of the high-redshift $\mathrm{SNe}$ Ia to suffer measurable reddening. A number of objects in the nearby sample display moderate 
extinction, for which we make individual corrections. We also include the Hubble constant as a free parameter in each of our fits to the other cosmological parameters. Treating the nearby sample in the same way as the distant sample is a crucial requirement of this work. Our experience observing the nearby sample aids our ability to accomplish this goal.

The statistics of gravitational lenses provide an alternate method for constraining the cosmological constant (Turner 1990; Fukugita, Futamase, \& Kasai 1990). Although current gravitational-lensing limits for the cosmological constant in a flat universe $\left(\Omega_{\Lambda} \leq 0.66\right.$ at $95 \%$ confidence; Kochanek 1996) are not inconsistent with these results, they are uncomfortably close. Future analysis that seeks to limit systematic uncertainties affecting both experiments should yield meaningful comparisons. The most incisive independent test may come from measurements of the fluctuation spectrum of the cosmic microwave background. While the supernova measurements provide a good constraint on $\Omega_{M}-\Omega_{\Lambda}$, the CMB measurements of the angular scale for the first Doppler peak, referring to much earlier epochs, are good measures of $\Omega_{M}+\Omega_{\Lambda}$ (White $\&$ Scott 1996). Since these constraints are nearly orthogonal in the coordinates of Figures 6 and 7, the region of intersection could be well defined. Ongoing experiments from balloons and the South Pole may provide the first clues to the location of that intersection.

Our detection of a cosmological constant is limited not by statistical errors but by systematic ones. Further intensive study of SNe Ia at low $(z<0.1)$, intermediate $(0.1 \leq z \leq 0.3)$, and high $(z>0.3)$ redshifts is needed to uncover and quantify lingering systematic uncertainties in this striking result.

\section{CONCLUSIONS}

1. We find the luminosity distances to well-observed $\mathrm{SNe}$ with $0.16 \leq z \leq 0.97$ measured by two methods to be in excess of the prediction of a low mass density $\left(\Omega_{M} \approx 0.2\right)$ universe by 0.25 to $0.28 \mathrm{mag}$. A cosmological explanation is provided by a positive cosmological constant with $99.7 \%$ $(3.0 \sigma)$ to more than $99.9 \%(4.0 \sigma)$ confidence using the complete spectroscopic SN Ia sample and the prior belief that $\Omega_{M} \geq 0$.

2. The distances to the spectroscopic sample of SNe Ia measured by two methods are consistent with a currently accelerating expansion $\left(q_{0} \leq 0\right)$ at confidence levels from $99.5 \%(2.8 \sigma)$ to more than $99.9 \%(3.9 \sigma)$ for $q_{0} \equiv\left(\Omega_{M} / 2\right)$ $-\Omega_{\Lambda}$ using the prior that $\Omega_{M} \geq 0$.
3. The data favor eternal expansion as the fate of the universe at the $99.7 \%(3.0 \sigma)$ to more than $99.9 \%(4.0 \sigma)$ confidence level from the spectroscopic SN Ia sample and the prior that $\Omega_{M} \geq 0$.

4. We estimate the dynamical age of the universe to be $14.2 \pm 1.7 \mathrm{Gyr}$ including systematic uncertainties in the zero point of the current Cepheid distance scale used for the host galaxies of three nearby SNe Ia (Saha et al. 1994, 1997).

5. These conclusions do not depend on inclusion of SN 1997ck $(z=0.97)$, whose spectroscopic classification remains uncertain, nor do they depend on which of two light curve fitting methods is used to determine the SN Ia distances.

6. The systematic uncertainties presented by gray extinction, sample selection bias, evolution, a local void, weak gravitational lensing, and sample contamination currently do not provide a convincing substitute for a positive cosmological constant. Further studies are needed to determine the possible influence of any remaining systematic uncertainties.

We wish to thank Alex Athey and S. Elizabeth Turner for their help in the supernova search at CTIO. We have benefited from helpful discussions with Peter Nugent, Alex Kim, Gordon Squires, and Marc Davis and from the efforts of Alan Dressler, Aaron Barth, Doug Leonard, Tom Matheson, Ed Moran, and Di Harmer. The work at U. C. Berkeley was supported by the Miller Institute for Basic Research in Science, by NSF grant AST 94-17213, and by grant GO-7505 from the Space Telescope Science Institute, which is operated by the Association of Universities for Research in Astronomy (AURA), Inc., under NASA contract NAS 5-26555. Support for A. C. was provided by the National Science Foundation through grant GF-1001-95 from AURA, Inc., under NSF cooperative agreement AST 8947990 and AST 96-17036, and from Fundación Antorchas Argentina under project A-13313. This work was supported at Harvard University through NSF grants AST 92-21648, AST 95-28899, and an NSF Graduate Research Fellowship. C. S. acknowledges the generous support of the Packard Foundation and the Seaver Institute. This research was based in part on spectroscopic observations obtained with the Multiple Mirror Telescope, a facility operated jointly by the Smithsonian Institution and the University of Arizona.

\section{APPENDIX}

\section{THE MULTICOLOR LIGHT CURVE SHAPE METHOD}

Following the success of Phillips (1993), Riess et al. (1995) employed a linear estimation algorithm (Rybicki \& Press 1992) to determine the relationship between the shape of a SN Ia light curve and its peak luminosity. This method was extended (Riess et al. 1996a) to utilize the SN Ia color curves to quantify the amount of reddening by interstellar extinction. In this appendix we describe further refinements and optimization of the MLCS method for the application to high-redshift SNe Ia.

Previously, the MLCS relations were derived from a set similar to the nearby ( $c z \leq 2000 \mathrm{~km} \mathrm{~s}^{-1}$ ) sample of Phillips (1993) (Riess et al. 1995, 1996a). The relative luminosities of this "training set" of SNe Ia were calibrated with independent distance indicators (Tonry 1991; Pierce 1994). The absolute SN Ia luminosities were measured from Cepheid variables populating the host galaxies (Saha et al. 1994, 1997). Yet at moderate distances, the most reliable distance indicator available in nature is the redshift. The recent harvest of SN Ia samples (Hamuy et al. 1996a; Riess et al. 1998c) with $c z \geq 2500 \mathrm{~km} \mathrm{~s}^{-1}$ provides a homogeneous training set of objects for MLCS with well-understood relative luminosities. Here we employ a set (see Table 10) of $B$ and $V$ light curves with $c z \geq 2500 \mathrm{~km} \mathrm{~s}^{-1}$ to determine the MLCS relations. 
TABLE 10

NeArby MLCS AND Template-Fitting SN Ia Parameters ${ }^{\mathrm{a}}$

\begin{tabular}{|c|c|c|c|c|c|c|c|c|}
\hline \multirow[b]{2}{*}{$\mathrm{SN}$} & \multirow[b]{2}{*}{$\log c z$} & \multicolumn{3}{|c|}{ MLCS } & \multicolumn{3}{|c|}{ Template Fitting } & \multirow{2}{*}{$\begin{array}{l}\text { HosT } \\
\text { TYPE }\end{array}$} \\
\hline & & $\Delta$ & $A_{B}$ & $\mu_{0}(\sigma)$ & $\Delta m_{15}(B)$ & $A_{B}$ & $\mu_{0}(\sigma)$ & \\
\hline 1992bo. & 3.734 & 0.31 & 0.00 & $34.72(0.16)$ & 1.59 & 0.01 & $34.88(0.21)$ & $\mathrm{E}$ \\
\hline $1992 b c$. & 3.779 & -0.50 & 0.00 & $34.87(0.11)$ & 0.88 & 0.00 & $34.77(0.15)$ & $\mathrm{L}$ \\
\hline $1992 \mathrm{aq} . . . .$. & 4.481 & 0.05 & 0.00 & $38.41(0.15)$ & 1.12 & 0.18 & $38.33(0.23)$ & $\mathrm{L}$ \\
\hline $1992 \mathrm{ae} . . . .$. & 4.350 & -0.05 & 0.00 & $37.80(0.17)$ & 1.21 & 0.28 & $37.77(0.19)$ & $\mathrm{E}$ \\
\hline $1992 P \ldots \ldots$ & 3.896 & -0.19 & 0.00 & $35.76(0.13)$ & 0.94 & 0.14 & $35.59(0.16)$ & $\mathrm{L}$ \\
\hline 1990af. & 4.178 & 0.09 & 0.18 & $36.53(0.15)$ & 1.66 & 0.16 & $36.67(0.25)$ & $\mathrm{L}$ \\
\hline $1994 \mathrm{M}$ & 3.859 & 0.04 & 0.08 & $35.39(0.18)$ & 1.47 & 0.06 & $35.49(0.20)$ & $\mathrm{E}$ \\
\hline 1994S .. & 3.685 & -0.44 & 0.00 & $34.27(0.12)$ & 1.02 & 0.03 & $34.34(0.14)$ & $\mathrm{L}$ \\
\hline 1994T ....... & 4.030 & 0.11 & 0.22 & $36.19(0.21)$ & 1.35 & 0.19 & $36.50(0.20)$ & $\mathrm{L}$ \\
\hline 1995D ...... & 3.398 & -0.42 & 0.00 & 33.01(0.13) & 0.96 & 0.23 & $32.79(0.16)$ & $\mathrm{E}$ \\
\hline 1995E ....... & 3.547 & -0.61 & 2.67 & $33.60(0.17)$ & 1.03 & 2.47 & $33.73(0.17)$ & $\mathrm{L}$ \\
\hline $1995 \mathrm{ac} . . . \ldots$ & 4.166 & -0.47 & 0.00 & $36.85(0.13)$ & 0.99 & 0.22 & $36.60(0.16)$ & $\mathrm{L}$ \\
\hline 1995ak ....... & 3.820 & 0.15 & 0.00 & $35.15(0.16)$ & 1.28 & 0.06 & $35.43(0.18)$ & $\mathrm{L}$ \\
\hline $1995 b d \ldots . .$. & 3.679 & -0.29 & 2.52 & $34.15(0.19)$ & 0.87 & 2.67 & $34.00(0.18)$ & $\mathrm{L}$ \\
\hline 1996C ....... & 3.924 & -0.07 & 0.24 & $35.98(0.20)$ & 0.97 & 0.42 & $35.82(0.20)$ & $\mathrm{L}$ \\
\hline $1996 a b \ldots . .$. & 4.572 & -0.13 & 0.00 & 39.01(0.13) & 1.10 & 0.09 & $39.10(0.17)$ & $\mathrm{L}$ \\
\hline $1992 \mathrm{ag} . . . .$. & 3.891 & -0.50 & 0.77 & $35.37(0.23)$ & 1.12 & 0.09 & $35.53(0.20)$ & $\mathrm{L}$ \\
\hline 1992al ...... & 3.625 & -0.35 & 0.00 & $33.92(0.11)$ & 1.13 & 0.00 & $34.13(0.14)$ & L \\
\hline $1992 b g . . . .$. & 4.024 & -0.06 & 0.50 & $36.26(0.21)$ & 1.14 & 0.44 & $36.49(0.21)$ & $\mathrm{L}$ \\
\hline $1992 b h \ldots . .$. & 4.130 & -0.16 & 0.28 & $36.91(0.17)$ & 1.05 & 0.34 & $36.87(0.17)$ & $\mathrm{L}$ \\
\hline $1992 b l \ldots \ldots$ & 4.111 & -0.06 & 0.00 & $36.26(0.15)$ & 1.50 & 0.00 & $36.53(0.20)$ & L \\
\hline $1992 b p \ldots \ldots$ & 4.379 & -0.26 & 0.04 & $37.65(0.13)$ & 1.27 & 0.03 & $37.96(0.15)$ & $\mathrm{E}$ \\
\hline $1992 b r \ldots \ldots$ & 4.418 & 0.40 & 0.00 & $38.21(0.19)$ & 1.77 & 0.02 & $38.09(0.36)$ & $\mathrm{E}$ \\
\hline $1992 b s \ldots .$. & 4.283 & 0.00 & 0.00 & 37.61(0.14) & 1.10 & 0.24 & $37.63(0.18)$ & L \\
\hline $1993 \mathrm{H} . . . .$. & 3.871 & 0.16 & 0.67 & $35.20(0.26)$ & 1.59 & 0.72 & $35.23(0.25)$ & $\mathrm{L}$ \\
\hline $19930 \ldots \ldots$ & 4.189 & 0.03 & 0.00 & $37.03(0.12)$ & 1.18 & 0.00 & $37.31(0.14)$ & $\mathrm{E}$ \\
\hline 1993ag ...... & 4.177 & -0.19 & 0.64 & $36.80(0.17)$ & 1.29 & 0.55 & $37.11(0.19)$ & $\mathrm{E}$ \\
\hline
\end{tabular}

${ }^{\text {a }}$ Light curves restricted to $B$ and $V$ data within 40 days of $B$ maximum.

${ }^{\mathrm{b}} \mathrm{E}=$ early-type host, $\mathrm{L}=$ late-type host.

The significant increase in the size of the available training set of SNe Ia since Riess et al. (1996a) supports an expansion of our description of the MLCS relations. Riess et al. (1996a) described SNe Ia light curves as a linear family of the peak luminosity:

$$
\begin{gathered}
\boldsymbol{m}_{V}=\boldsymbol{M}_{V}+\boldsymbol{R}_{V} \Delta+\mu_{V}, \\
\boldsymbol{m}_{B-V}=\boldsymbol{M}_{B-V}+\boldsymbol{R}_{B-V} \Delta+E_{B-V},
\end{gathered}
$$

where $\boldsymbol{m}_{V}$ and $\boldsymbol{m}_{B-V}$ are the observed light and color curves, $\Delta \equiv M_{v}-M_{v}$ (standard) is the difference in maximum luminosity between the fiducial template SN Ia and any other SN Ia, $\boldsymbol{R}_{V}$ and $\boldsymbol{R}_{B-V}$ are vectors of correlation coefficients between $\Delta$ and the light curve shape, $\mu_{V}$ is the apparent distance modulus, and $E_{B-V}$ is the color excess. All symbols in boldface denote vectors that are functions of SN Ia age, with $t=0$ taken by convention as the epoch of $B$ maximum.

By adding a second-order term in the expansion, our empirical model becomes

$$
\begin{gathered}
\boldsymbol{m}_{V}=\boldsymbol{M}_{V}+\boldsymbol{R}_{V} \Delta+\boldsymbol{Q}_{V} \Delta^{2}+\mu_{V}, \\
\boldsymbol{m}_{B-V}=\boldsymbol{M}_{B-V}+\boldsymbol{R}_{B-V} \Delta+\boldsymbol{Q}_{B-V} \Delta^{2}+E_{B-V},
\end{gathered}
$$

where $Q_{V}, Q_{B-V}$ are the correlation coefficients of the quadratic relationship between $\Delta^{2}$ and the light curve shape. The vectors of coefficients $\left(\boldsymbol{R}_{V}, \boldsymbol{R}_{B-V}, \boldsymbol{Q}_{V}, \boldsymbol{Q}_{B-V}\right)$, as well as the fiducial templates $\left(\boldsymbol{M}_{V}, \boldsymbol{M}_{B-V}\right)$, are determined from the training set of SNe Ia listed in Table 10. The empirical light and color curve families are shown in Figure 13. As before, these MLCS relations show that the more luminous SNe Ia have broader light curves and are bluer until day $\sim 35$, by which time all $\mathrm{SNe}$ Ia have the same color. The primary difference from the previous MLCS relations is that near maximum, the color range spanned by the same range of SN Ia luminosities is much reduced. Further, the quadratic MLCS relations reveal that SNe Ia that are brighter or dimmer (than the fiducial value) by equal amounts do not show equal changes in their colors. Faint $\mathrm{SNe}$ Ia are far redder than the amount by which luminous SNe Ia are blue.

Fitting of this quadratic model (eqs. [A3]-[A4]) to a SN Ia still requires the determination of four "free " parameters: $\Delta, \mu_{V}$, $E_{B-V}$, and $t_{\max }$. The parameters are determined by minimizing the expected deviations between data and model:

$$
\chi^{2}=\boldsymbol{r}_{x} \mathbf{C}^{-1} \boldsymbol{r}_{x}^{T}
$$

where

$$
\boldsymbol{r}_{x}=\boldsymbol{m}_{x}-\boldsymbol{M}_{x}-\boldsymbol{R}_{x} \Delta-\boldsymbol{Q}_{x} \Delta^{2}-\mu_{x}
$$




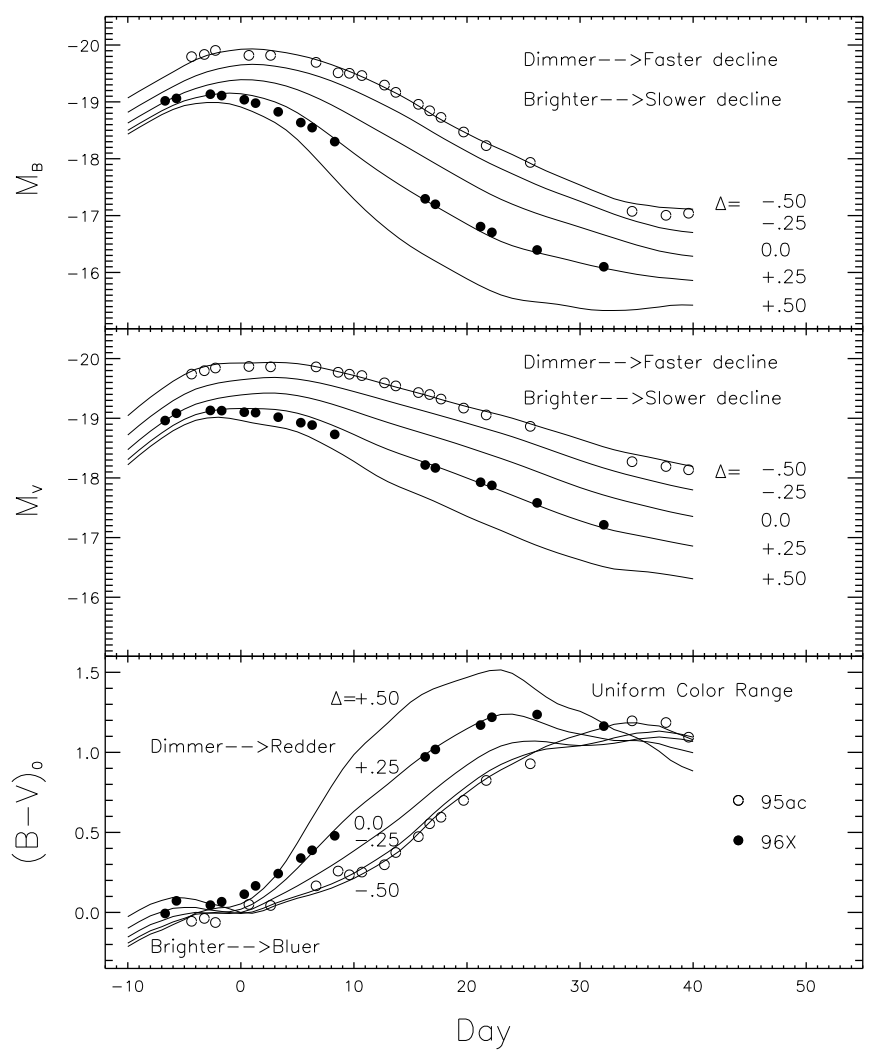

FIG. 13.-MLCS empirical SN Ia light curve families in $\mathrm{M}_{B}, M_{V}$, and $(B-V)_{0}$. The derived light curves are given as a function of the luminosity difference, $\Delta$, between the peak visual luminosity of a SN Ia and a fiducial $(\Delta=0)$ SN Ia. Properties of the SN Ia families are indicated in the figure and the Appendix. The light and color curves of SN 1995ac (open symbols) and SN 1996X (filled symbols) are overplotted as examples of luminous and dim SNe Ia, respectively.

for any band $x$. Here $\mathbf{C}$ is the correlation matrix of the model and the measurements. Correlations of the data from the model were determined from the SNe Ia of Table 10. These correlations result from our still imperfect (but improving) description of the light curve shape behavior. Future expansion of the model will reduce these correlations further until they become constraints on the unpredictable, turbulent behavior of the SN Ia atmosphere. Riess et al. (1996a) quantified the autocorrelation (diagonal matrix elements) of the linear model. Here we have determined, in addition, the covariance (off-diagonal matrix elements) between two measurements of differing SN Ia age, passband, or both. The correlation matrix of the measurements, commonly called the "noise," is, as always, provided by the conscientious observer.

The a priori values for $\Delta$ used to determine the vectors $R_{V}, R_{B-V}, Q_{V}, Q_{B-V}, M_{V}$, and $M_{B-V}$ are the differences between the measured peak magnitudes and those predicted by the SN Ia host galaxy redshift. These values for $\Delta$ must be corrected for the extinction, $A_{V}$. Because the values of $A_{V}$ are not known a priori, we use an initial guess derived from the color excess measured from the uniform color range of SNe Ia after day 35 (Riess et al. 1996a; Lira 1995).

Initial guesses for $\Delta, \mu_{V}, E_{B-V}$, and $t_{\max }$ yield estimates for $\boldsymbol{R}_{V}, \boldsymbol{R}_{B-V}, \boldsymbol{Q}_{V}, \boldsymbol{Q}_{B-V}, \boldsymbol{M}_{V}$, and $\boldsymbol{M}_{B-V}$ by minimizing equation (A5) with respect to the latter. These estimates for $\boldsymbol{R}_{V}, \boldsymbol{R}_{B-V}, \boldsymbol{Q}_{V}, \boldsymbol{Q}_{B-V}, \boldsymbol{M}_{V}$, and $\boldsymbol{M}_{\boldsymbol{B}-\boldsymbol{V}}$ yield improved estimates of $\Delta, \mu_{V}$, $E_{B-V}$, and $t_{\max }$ also determined by minimizing equation (A5) with respect to the latter. This iterative determination of these vectors and parameters is repeated until convergence is reached. Subsequent determination of the parameters $\Delta, \mu_{V}, E_{B-V}$, and $t_{\max }$ for SNe Ia not listed in Table 10 (such as those reported here) is done by using the fixed vectors derived from this training process.

We also employ a refined estimate of the selective absorption to color excess ratio, $R_{V}=A_{V} / E_{B-V}$, which has been calculated explicitly as a function of SN Ia age from accurate spectrophotometry of SNe Ia (Nugent, Kim, \& Perlmutter 1998a). This work shows that although $R_{V}$ is the canonical value of $\sim 3.1$ for SNe Ia at maximum light or before, over the first 10 days after maximum $R_{V}$ slowly rises to about 3.4. For highly reddened SNe Ia, this change in $R_{V}$ over time can appreciably affect the shape of the SN Ia light curve(Leibundgut 1989).

Finally, we have refined our a priori understanding of the likelihood for SN Ia interstellar extinction from host galaxies. The previous incarnation of MLCS (Riess et al. 1996a) employed a "Bayesian filter" to combine our measurement of extinction with our prior knowledge of its one-directional effect. In addition, it is less probable to observe a very large amount of extinction due to the finite column density of a spiral disk as well as a reduced likelihood for detection of SNe with large extinctions. To quantify this a priori likelihood for extinction we have adopted the calculations of Hatano et al. (1998), who determined the extinction distribution for SNe Ia in the bulge and disk of late-type galaxies. The primary difference between our previous a priori distribution and the results of Hatano et al. (1998) are that nontrivial quantities of extinction are even 
less probable than assumed. In particular, Hatano et al. (1998) show that two-thirds of SNe Ia suffer less than 0.3 to 0.5 mag of extinction, which is approximately half the amount of extinction previously assumed. Despite our use of an externally derived Bayesian prior for probable SN Ia extinction, it is important to continue testing that the a posteriori extinction distribution matches the expected one. A statistically significant departure could imply an important deficiency in the SN Ia luminosity, light curve shape, and color relations. Specifically, excessively blue SNe Ia such as SN 1994D $\left(E_{B-V} \approx-0.10 \pm 0.04\right)$, if common, would reveal a shortcoming of these MLCS relations. However, using the current MLCS relations, the best estimate we can make for such blue SNe Ia is that their extinctions are negligible. (We note, however, that as the high- and low-redshift sample size increases, a better estimator of the extinction is the mean and not the mode of the extinction). If the a priori distributions of Hatano et al. (1998) are not significantly in error, this practice is statistically sensible and does not introduce a significant distance bias.

Arnett, W. D. 1969, Ap\&SS, 5, 280

Bahcall, J. N., et al. 1996, ApJ, 457, 19

Bahcall, N. A., Fan, X., \& Cen, R. 1997, ApJ, 485, 53

Bolte, M., \& Hogan, C. J. 1995, Nature, 376, 399

Bouchet, P., Lequeux, J., Maurice, E., Prévot, L., \& Prévot-Burnichon, M. L. 1985, A\&A, 149, 300

Branch, D. 1998, ARA\&A, in press

Branch, D., Fisher, A., \& Nugent, P. 1993, AJ, 106, 2383

Branch, D., \& Miller, D. 1993, ApJ, 405, L5

Branch, D., \& Tammann, G. A. 1992, ARA\&A, 30, 359

Branch, D., \& van den Bergh, S. 1993, AJ, 105, 2251

Branch, D., et al. 1988, ApJ, 330, 117

Burstein, D., \& Heiles, C. 1982, AJ, 87, 1165

Caldwell, R. R., Dave, R., \& Steinhardt, P. J. 1998, Phys. Rep. Lett., 80, 1582

Cappellaro, E., et al. 1997, A\&A, 322, 431

Cardelli, J. A., Clayton, G. C., \& Mathis, J. S. 1989, ApJ, 345, 245

Carlberg, R. G., Yee, H. K. C., Ellingson, E., Abraham, R., Gravel, P., Morris, S., \& Pritchet, C. J. 1996, ApJ, 462, 32

Carroll, S. M., Press, W. H., \& Turner, E. L. 1992, ARA\&A, 30, 499

Chaboyer, B. 1995, ApJ, 444, L9

Chaboyer, B., Demarque, P., Kernan, P. J., \& Krauss, L. M. 1998, ApJ, 494,96

Chaboyer, B., Demarque, P., \& Sarajedini, A. 1996, ApJ, 459, 558

Clocchiatti, A., et al. 1998, in preparation

Colgate, S. 1979, ApJ, 232, 404

Colgate, S., \& McKee, C. 1969, ApJ, 157, 623

Cowan, J. J., McWilliam, A., Sneden, C., \& Burris, D. L. 1997, ApJ, 480, 246

Cristiani, S., et al. 1992, A\&A, 259, 63

Della Valle, M., \& Panagia, N. 1992, AJ, 104, 696

Doggett, J. B., \& Branch, D. 1985, AJ, 90, 2303

Feast, M. W., \& Catchpole, R. M. 1997, MNRAS, 286, 1P

Feast, M. W., \& Walker, A. R. 1987, ARA\&A, 25, 345

Filippenko, A. V. 1997, ARA\&A, 35, 309

Filippenko, A. V., et al. 1992a, ApJ, 384, 15

$$
\text { 1992b, AJ, 104, } 1543
$$$$
1998, \text { ApJ, in press }
$$

Ford, C. 1998, ApJ, in press

Fukugita, M., Futamase, T., \& Kasai, M. 1990, MNRAS, 246, 24

Garnavich, P., et al. 1996a, IAU Circ. 6332

.1996b, IAU Circ. 6358 1996c, IAU Circ. 6861

1998a, ApJ, 493, 53

. 1998b, ApJ, in press

Goobar, A., \& Perlmutter, S. 1995, ApJ, 450, 14

Gratton, R. G., Fusi Pecci, F., Carretta, E., Clementini, G., Corsi, C. E., \& Lattanzi, M. 1997, ApJ, 491, 749

Hamuy, M., et al. 1994, AJ, 108, 2226

.1993a, AJ, 106, 2392 1996a, AJ, 112, 2408

Hamuy, M., Phillips, M. M., Maza, J., Suntzeff, N. B., Schommer, R. A., \& Avilés, R. 1995, AJ, 109, 1

Hamuy, M., Phillips, M. M., Schommer, R. A., Suntzeff, N. B., Maza, J., \& Avilés, R. 1996b, AJ, 112, 2391

Hamuy, M., Phillips, M. M., Suntzeff, N. B., Schommer, R. A., Maza, J., \& Avilés, R. 1996c, AJ, 112, 2398

Hamuy, M., Phillips, M. M., Suntzeff, N. B., Schommer, R. A., Maza, J., Smith, R. C., Lira, P., \& Avilés, R. 1996d, AJ, 112, 2438

Hamuy, M., Phillips, M. M., Wells, L. A., \& Maza, J. 1993b, PASP, 105, 787

Hamuy, M., \& Pinto, P. 1998, in preparation

Hansen, L., Jørgensen, H. E., \& Nørgaard-Nielsen, H. U. 1987, Messenger, 47,46

Hatano, K., Branch, D., \& Deaton, J. 1998, ApJ, 502, 177

Hodge, P. W.., \& Kennicutt, R. C. 1982, AJ, 87, 264

Höflich, P., Wheeler, J. C., \& Thielemann, F. K. 1998, ApJ, 495, 617

Holz, D. E., \& Wald, R. M. 1998, Phys. Rev. D, in press

Hoyle, F., \& Fowler, W. A. 1960, ApJ, 132, 565

\section{REFERENCES}

Huang, J. S., Cowie, L. L., Gardner, J. P., Hu, E. M., Songaila, A., \& Wainscoat, R. J. 1997, ApJ, 476, 12

Kantowski, R., Vaughan, T., \& Branch, D. 1995, ApJ, 447, 35

Kim, A., Goobar, A., \& Perlmutter, S. 1996, PASP, 108, 190

Kim, A., et al. 1997, ApJ, 476, 63

Kirshner, R. P., et al. 1995, IAU Circ. 6267

Kochanek, C. S. 1996, ApJ, 466, 638 1997, ApJ, 491, 13

Kowal, C. T. 1968, AJ, 73, 1021

Landolt, A. U. 1992, AJ, 104, 340

Leibundgut, B. 1989, Ph.D. thesis, Basel Univ.

Leibundgut, B., et al. 1993, AJ, 105, 301

Lin, H., et al. 1996, ApJ, 471, 617

Lira, P. 1995, Master's thesis, Univ. Chile

Lupton, R. 1993, Statistics in Theory and Practice (Princeton: Princeton Univ. Press)

Mateo, M., \& Schechter, P. L. 1989, in Proc. First ESO/ST-ECF Workshop on Data Analysis, ed. P. J. Grosbøl, F. Murtaugh, \& R. H. Warmels (Garching: ESO), 127

Madore, B. F., \& Freedman, W. L. 1991, PASP, 103, 933

. 1998, ApJ, 492, 110

Marzke, R. O., Geller, M. J., da Costa, L. N., \& Huchra, J. P. 1995, AJ, 110, 477

Menzies, J. W. 1989, MNRAS, 237, 21

Miller, D., \& Branch, D. 1990, AJ, 100, 530

Nørgaard-Nielsen, H., et al. 1989, Nature, 339, 523

Nugent, P., Kim, A., \& Perlmutter, S. 1998a, in preparation $1998 \mathrm{~b}$, in preparation

1998c, IAU Circ. 6804

Nugent, P., Phillips, M., Baron, E., Branch, D., \& Hauschildt, P. 1995, ApJ, 455, L147

Oke, J. B., \& Sandage, A. 1968, ApJ, 154, 21

Oke, J. B., et al. 1995, PASP, 107, 375

Oswalt, T. D., Smith, J. A., Wood, M. A., \& Hintzen, P. 1996, Nature, 382, 692

Perlmutter, S., et al. 1995, ApJ, 440, 41

. 1997, ApJ, 483, 565

Phillips, M. M. 1993, ApJ, L105, 413

Phillips, M. M., et al. 1987, PASP, 99, 592 1998 , in preparation

Phillips, M. M., Wells, L., Suntzeff, N., Hamuy, M., Leibundgut, B., Kirshner, R. P., \& Foltz, C. 1992, AJ, 103, 1632

Pierce, M. J. 1994, ApJ, 430, 53

Porter, A. C., \& Filippenko, A. V. 1987, AJ, 93, 1372

Pskovskii, Y. 1984, Soviet Astron., 28, 658

Reid, I. N. 1997, AJ, 114, 161

Renzini, A., et al. 1996, ApJ, 465, L23

Reiss, D. J., Germany, L. M., Schmidt, B. P., \& Stubbs, C. W. 1998a, AJ, 115,26

Riess, A. G. 1996, Ph.D. thesis, Harvard Univ.

Riess, A. G., et al. 1997, AJ, 114, 722

Riess, A. G., Nugent, P. E., Filippenko, A. V., Kirshner, R. P., \& Perlmutter, S. 1998b, ApJ, 504, 935

Riess, A. G., et al. 1998c, in preparation

Riess, A. G., Press, W. H., \& Kirshner, R. P. 1995, ApJ, 438, L17 1996a, ApJ, 473, 88 $1996 \mathrm{~b}$, ApJ, 473, 588

Rood, H. J. 1994, PASP, 106, 170

Ruiz-Lapuente, P., Cappellaro, E., Turatto, M., Gouiffes, C., Danziger, I. J., Della Valle, M., \& Lucy, L. B. 1992, ApJ, 387, L33

Rybicki, G. B., \& Press, W. H. 1992, ApJ, 398, 169

Saha, A., et al. 1994, ApJ, 425, 14 .1997, ApJ, 486, 1

Sandage, A., \& Tammann, G. A. 1993, ApJ, 415, 1

Sandage, A., et al. 1994, ApJ, 423, L13

Savage, B. D., \& Mathis, J. S. 1979, ARA\&A, 17, 73

Schechter, P. L., Mateo, M., \& Saha, A. 1993, PASP, 105, 1342

Schmidt, B. P., et al. 1998, ApJ, in press 
Strauss, M. A., \& Willick, J. A. 1995, Phys. Rep., 261, 271

Tammann, G. A., \& Leibundgut, B. 1990, A\&A, 236, 9

Tonry, J. L. 1991, ApJ, 373, L1

Tripp, R. 1997, A\&A, 325, 871 1998, A\&A, 331, 815

Turatto, M., Benetti, S., Cappellaro, E., Danziger, I. J., Della Valle, M., Gouiffes, C., Mazzali, P. A., \& Patat, F. 1996, MNRAS, 283, 1

Turner, E. L. 1990, ApJ, 365, 43

Uomoto, A., \& Kirshner, R. P. 1985, A\&A, 149, L7

VandenBerg, D. A., Stetson, P. B., \& Bolte, M. 1996, ARA\&A, 34, 461 von Hippel, T., Bothun, G. D., \& Schommer, R. A. 1997, AJ, 114, 1154

Walterbos, R. 1986, Ph.D. thesis, Leiden Univ.

Wambsganss, J., Cen, R., Xu, G., \& Ostriker, J. 1997, ApJ, 475, L81

Wheeler, J. C., \& Harkness, R. P. 1990, Rep. Prog. Phys., 53, 1467

Wheeler, J. C., Harkness, R. P., Barkat, Z., \& Swartz, D. 1986, PASP, 98, 1018

Wheeler, J. C., \& Levreault, R. 1985, ApJ, 294, L17

White, M., \& Scott, D. 1996, ApJ, 459, 415

Zehavi, I., Riess, A. G., Kirshner, R. P., \& Dekel, A. 1998, ApJ, 503, 483 